\title{
C-H Functionalization of Peptides via Cyclic Aminal Intermediates
}

\author{
Michael Kohr and Uli Kazmaier* \\ Saarland University, Organic Chemistry I, Campus, Building C4.2 \\ D-66123 Saarbrücken, Germany
}

Supporting Information

Table of Contents

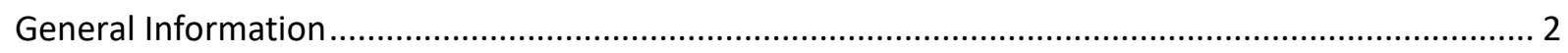

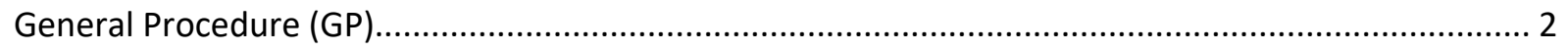

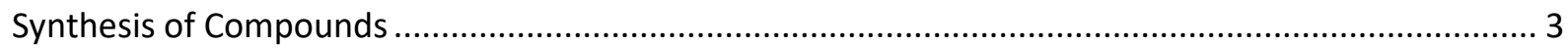

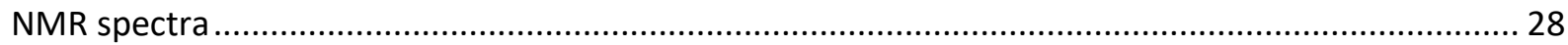




\section{General Information}

All air and moisture sensitive reactions were carried out in dried glassware $\left(>100^{\circ} \mathrm{C}\right)$ under nitrogen or argon atmosphere. THF was dried over sodium and distilled before use. The products were purified by automated column chromatography on silica columns (RediSep Rf, Teledyne Isco) or C18 columns (Telos). Mixtures of ethyl acetate (EtOAc), petroleum ether (PE, $40-60{ }^{\circ} \mathrm{C}$ fraction), cyclohexane, dichloromethane (DCM), methanol $(\mathrm{MeOH})$ or acetonitrile (MeCN) and water were generally used as eluents. Analytical TLC was performed on pre-coated silica gel plates (Machery-Nagel, Polygram Sil $\mathrm{G} / \mathrm{UV}_{254}$ ). Detection was accomplished with UV light (254 nm), $\mathrm{KMnO}_{4}$ solution, ninhydrin solution or ceric ammonium sulfate solution. Compounds were occasionally freeze-dried with a Christ lyophiliser. Melting points were detected with a MEL-TEMP II (Laboratory devices) apparatus and are uncorrected. ${ }^{1} \mathrm{H}$ and ${ }^{13} \mathrm{C}$ NMR spectra were recorded with a Bruker AV400 $\left[400 \mathrm{MHz}\left({ }^{1} \mathrm{H}\right)\right.$ and $\left.100 \mathrm{MHz}\left({ }^{13} \mathrm{C}\right)\right]$ or a Bruker AV500 $\left[500 \mathrm{MHz}\left({ }^{1} \mathrm{H}\right)\right.$ and $\left.125 \mathrm{MHz}\left({ }^{13} \mathrm{C}\right)\right]$ spectrometer. For some rotamer containing samples the NMR spectra were measured at $373 \mathrm{~K}$. Chemical shifts are reported in ppm relative to TMS or internal solvent signal. Mass spectra were recorded with a Finnigan MAT 95 spectrometer (quadropole) using the $\mathrm{Cl}$ technique. Optical rotations were measured with a Perkin-Elmer polarimeter (model 341 or 241$)$ in a thermostated $\left(20^{\circ} \mathrm{C} \pm 0.1^{\circ} \mathrm{C}\right)$ cuvette, using a sodium vapour lamp $(\lambda=589$ $\mathrm{nm})$ as radiation source. The concentrations are given in $\mathrm{g}$ per $100 \mathrm{~mL}$. LCMS analyses were accomplished on a Shimadzu (LC-10At, autoinjector SCL-6B, mass spectrometer LCMS-2020) with a Phenomenex Luna C18(2) column ( $50 \times 4.6 \mathrm{~mm}$, grain size $3 \mu \mathrm{m})$.

\section{General Procedure (GP)}

\section{GP: C-H activation of aminals}
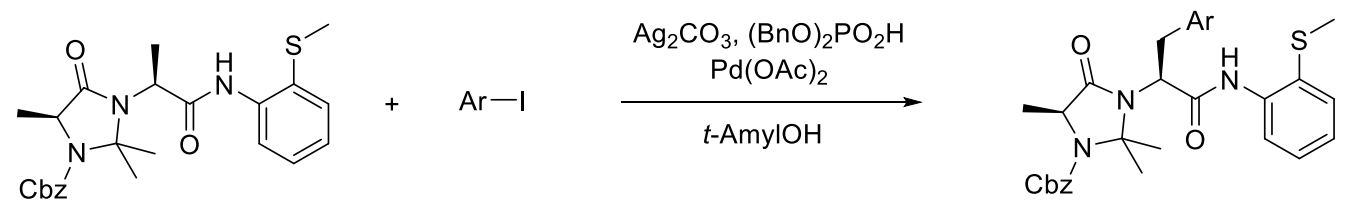

A $10 \mathrm{~mL}$ crimp vial was charged with the peptide compound, 2.0 equiv $\mathrm{Ag}_{2} \mathrm{CO}_{3}, 20$ mol\% $(\mathrm{BnO})_{2} \mathrm{PO}_{2} \mathrm{H}$, $10 \mathrm{~mol} \% \mathrm{Pd}(\mathrm{OAc})_{2}$ and 2.0 equiv of the electrophile. The reactants were suspended in tert-Amyl alcohol $(7.7 \mathrm{~mL} / \mathrm{mmol})$, a magnetic stirring bar was added and the vial or schlenk tube was sealed under argon atmosphere. The reaction mixture was heated in a heating block (alumina) and stirred at given temperature for 2-4 days. After cooling to room temperature, the solvent was evaporated and the crude product purified by column chromatography. 


\section{Synthesis of Compounds}

Benzyl (S)-2-(((S)-1-oxo-1-(quinolin-8-ylamino)propan-2-yl)carbamoyl)pyrrolidine-1-carboxylate (2)

HOBt (1.59 g, $10.4 \mathrm{mmol}, 1.1 \mathrm{eq}$ ) ) and EDC-HCl (1.99 g, $10.4 \mathrm{mmol}, 1.1 \mathrm{eq}$.) were added successively to a solution of Cbz-L-Pro-OH (2.35 g, $9.44 \mathrm{mmol}, 1.0$ eq.) and 2-Amino- $N$-(quinolin-8-yl)propenamide 1 $\left(2.16 \mathrm{~g}, 10.0 \mathrm{mmol}, 1.06\right.$ eq.) in $\mathrm{DCM}(94 \mathrm{~mL})$ at $0{ }^{\circ} \mathrm{C}$. The reaction mixture was warmed to room temperature overnight before being hydrolyzed with aqueous $\mathrm{HCl}$ solution (1M). The mixture was extracted twice with $\mathrm{DCM}$ and the combined organics were washed successivelywith saturated $\mathrm{NaHCO}_{3}$ solution and dried over $\mathrm{Na}_{2} \mathrm{SO}_{4}$. After filtration, the solvent was evaporated and the crude product was purified by column chromatography $\left(\mathrm{SiO}_{2}, \mathrm{DCM}: \mathrm{MeOH} 98: 2\right)$ to afford dipeptide 2 (3.75 $\mathrm{g}, 8.40 \mathrm{mmol}, 89 \%)$ as a white solid.

$\mathbf{R}_{\mathbf{f}}(\mathbf{2})=0.55\left(\mathrm{SiO}_{2}, \mathrm{DCM}: \mathrm{MeOH} 95: 5\right)$

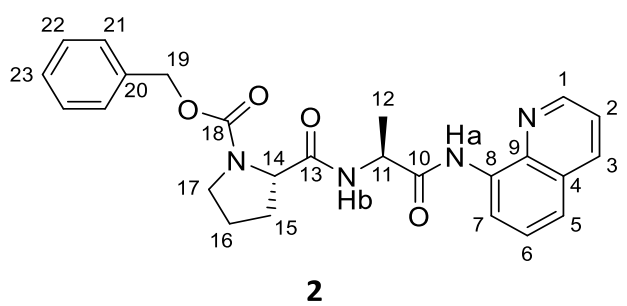

${ }^{1} \mathrm{H}-\mathrm{NMR}\left(500 \mathrm{MHz}\right.$, DMSO-d $\left.\mathrm{d}_{6}, \mathbf{3 7 3 K}\right): \delta=1.39\left(\mathrm{~d},{ }^{3} \mathrm{~J}_{12,11}=6.3 \mathrm{~Hz}, 3 \mathrm{H}, 12-\mathrm{H}\right), 1.87\left(\mathrm{~m}, 1 \mathrm{H}, 16-\mathrm{H}_{\mathrm{a}}\right), 1.95$ $\left(\mathrm{m}, 1 \mathrm{H}, 16-\mathrm{H}_{\mathrm{b}}\right), 2.24\left(\mathrm{~m}, 1 \mathrm{H}, 15-\mathrm{H}_{\mathrm{a}}\right), 2.31\left(\mathrm{~m}, 1 \mathrm{H}, 15-\mathrm{H}_{\mathrm{b}}\right), 3.49(\mathrm{~m}, 2 \mathrm{H}, 17-\mathrm{H}), 4.41\left(\mathrm{dd},{ }^{3} \mathrm{~J}_{14,15 \mathrm{~b}}=8.2 \mathrm{~Hz}\right.$, $\left.{ }^{3} J_{14,15 a}=3.8 \mathrm{~Hz}, 1 \mathrm{H}, 14-\mathrm{H}\right), 4.54\left(\mathrm{dq},{ }^{3} J_{11, \mathrm{NHb}}=7.2 \mathrm{~Hz},{ }^{3} J_{11,12}=6.3 \mathrm{~Hz}, 1 \mathrm{H}, 11-\mathrm{H}\right), 5.04\left(\mathrm{~d},{ }^{2} J_{19 a, 19 b}=12.9\right.$ $\left.\mathrm{Hz}, 1 \mathrm{H}, 19-\mathrm{H}_{\mathrm{a}}\right), 5.09\left(\mathrm{~d},{ }^{2} \mathrm{~J}_{19 \mathrm{~b}, 19 \mathrm{a}}=12.9 \mathrm{~Hz}, 1 \mathrm{H}, 19-\mathrm{H}_{\mathrm{b}}\right), 7.27-7.36(\mathrm{~m}, 5 \mathrm{H}, 21-\mathrm{H} / 22-\mathrm{H} / 23-\mathrm{H}), 7.57$ (t, $\left.{ }^{3} J_{6,5}=8.2 \mathrm{~Hz},{ }^{3} J_{6,7}=7.5 \mathrm{~Hz}, 1 \mathrm{H}, 6-\mathrm{H}\right), 7.61\left(\mathrm{dd},{ }^{3} J_{2,3}=8.2 \mathrm{~Hz},{ }^{3} J_{2,1}=4.1 \mathrm{~Hz}, 1 \mathrm{H}, 2-\mathrm{H}\right), 7.65\left(\mathrm{dd},{ }^{3} J_{5,6}=8.2\right.$ $\left.\mathrm{Hz},{ }^{4} J_{5,7}=1.3 \mathrm{~Hz}, 1 \mathrm{H}, 5-\mathrm{H}\right), 8.37\left(\mathrm{dd},{ }^{3} J_{3,2}=8.2 \mathrm{~Hz},{ }^{4} J_{3,1}=1.6 \mathrm{~Hz}, 1 \mathrm{H}, 3-\mathrm{H}\right), 8.37\left(\mathrm{bs}, 1 \mathrm{H}, \mathrm{N}-\mathrm{H}_{\mathrm{b}}\right), 8.63$ (dd, $\left.{ }^{3} J_{7,6}=7.5 \mathrm{~Hz},{ }^{4} J_{7,5}=1.3 \mathrm{~Hz}, 1 \mathrm{H}, 7-\mathrm{H}\right), 8.86\left(\mathrm{dd},{ }^{3} J_{1,2}=4.1 \mathrm{~Hz},{ }^{4} J_{1,3}=1.6 \mathrm{~Hz}, 1 \mathrm{H}, 1-\mathrm{H}\right), 10.24(\mathrm{bs}, 1 \mathrm{H}, \mathrm{N}-$ $\mathrm{H}_{\mathrm{a}}$ ).

${ }^{13} \mathrm{C}-N M R$ (125 MHz, DMSO-d 6 , 373K): $\delta=16.5$ (q, C-12), 22.9 (t, C-16), 30.1 (t, C-15), 46.4 (t, C-17), 49.3 (d, C-11), 59.1 (d, C-14), 65.5 (t, C-19), 115.5 (d, C-7), 121.3 (d, C-5), 121.5 (d, C-2), 126.4 (d, C-6), 126.7 (d, C-21), 127.0 (d, C-23), 127.3 (s, C-4), 127.7 (d, C-22), 133.6 (s, C-8), 136.0 (d, C-3), 136.5 (s, C-20), 137.7 (s, C-9), 148.0 (d, C-1), 153.5 (s, C-18), 170.4 (s, C-10), 172.0 (s, C-13).

Optical rotation: $\quad[\alpha]_{D}^{20}=-89.1\left[\mathrm{CHCl}_{3}, \mathrm{c}=1.00\right]$

Melting point: $\quad 150-152^{\circ} \mathrm{C}$

HRMS (Cl) m/z: [M + H] $]^{+}$Calcd for $\mathrm{C}_{25} \mathrm{H}_{27} \mathrm{~N}_{4} \mathrm{O}_{4}$ 447.2027; Found 447.2035

\section{(S)-2-((S)-1-Oxotetrahydro-1H-pyrrolo[1,2-c]imidazol-2(3H)-yl)- $\mathrm{N}$-(quinolin-8-yl)propenamide (3)}

Hydrogenation:

$\mathrm{Pd} / \mathrm{C}$ (360 mg, dry, $10 \mathrm{wt} \% \mathrm{Pd}$ ) was added to a solution of dipeptide 2 (3.64 g, $8.15 \mathrm{mmol}, 1.0$ eq.) in $\mathrm{MeOH}(41 \mathrm{~mL})$ and the suspension was stirred under hydrogen atmosphere (1 atm) for 4 hours. 
Subsequently, the catalyst was removed by filtration through Celite and the solvent thereafter removed in vacuo to afford the free amine $(2.45 \mathrm{~g}, 7.84 \mathrm{mmol}, 96 \%)$ as a yellow solid, which was used in the next step without further purification.

\section{Aminal formation:}

The previously prepared amine ( $350 \mathrm{mg}, 1.12 \mathrm{mmol}, 1.0$ eq.) was dissolved in formalin (1.2 mL, $37 \mathrm{wt} \%$ in water) and the mixture was refluxed in an oil bath for 30 minutes. The resulting solution was then extracted three times with DCM and the combined organic phases were dried over $\mathrm{Na}_{2} \mathrm{SO}_{4}$. Afterwards, the solvent was removed in vacuo and the crude product purified by column chromatography $\left(\mathrm{SiO}_{2}\right.$, DCM:MeOH 96:4 $\rightarrow$ 95:5) to afford imidazolidinone 3 (349 mg, $1.08 \mathrm{mmol}, 96 \%$ ) as a yellow resin.

$\mathbf{R}_{\mathbf{f}}(\mathbf{3})=0.56\left(\mathrm{SiO}_{2}, \mathrm{DCM}: \mathrm{MeOH} 90: 10\right)$

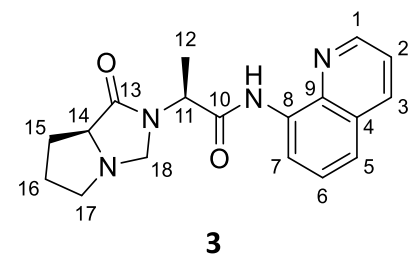

${ }^{1} \mathrm{H}-\mathrm{NMR}\left(400 \mathrm{MHz}, \mathrm{CDCl}_{3}\right): \delta=1.54\left(\mathrm{~d},{ }^{3} \mathrm{~J}_{12,11}=7.2 \mathrm{~Hz}, 3 \mathrm{H}, 12-\mathrm{H}\right), 1.84(\mathrm{~m}, 2 \mathrm{H}, 16-\mathrm{H}), 2.13(\mathrm{~m}, 2 \mathrm{H}, 15-$ H), $2.58\left(\mathrm{dt},{ }^{2} J_{17 \mathrm{a}, 17 \mathrm{~b}}=9.1 \mathrm{~Hz},{ }^{3} J_{17 \mathrm{a}, 16}=8.1 \mathrm{~Hz}, 1 \mathrm{H}, 17-\mathrm{H}_{\mathrm{a}}\right), 3.23\left(\mathrm{dt},{ }^{2} J_{17 \mathrm{~b}, 17 \mathrm{a}}=9.1 \mathrm{~Hz},{ }^{3} J_{17 \mathrm{~b}, 16}=5.3 \mathrm{~Hz}, 1 \mathrm{H}\right.$, $\left.17-\mathrm{H}_{\mathrm{b}}\right), 3.88\left(\mathrm{dd},{ }^{3} J_{14,15 \mathrm{a}}=8.9 \mathrm{~Hz},{ }^{3} J_{14,15 b}=4.4 \mathrm{~Hz}, 1 \mathrm{H}, 14-\mathrm{H}\right), 4.26\left(\mathrm{~d},{ }^{2} J_{18 \mathrm{a}, 18 \mathrm{~b}}=8.6 \mathrm{~Hz}, 1 \mathrm{H}, 18-\mathrm{H}_{\mathrm{a}}\right), 4.73$ $\left(\mathrm{d},{ }^{2} J_{18 b, 18 \mathrm{a}}=8.6 \mathrm{~Hz}, 1 \mathrm{H}, 18-\mathrm{H}_{\mathrm{b}}\right), 4.97\left(\mathrm{q},{ }^{3} J_{11,12}=7.2 \mathrm{~Hz}, 1 \mathrm{H}, 11-\mathrm{H}\right.$ ), $7.43\left(\mathrm{dd},{ }^{3} J_{2,3}=8.3 \mathrm{~Hz},{ }^{3} \mathrm{~L}_{2,1}=4.2 \mathrm{~Hz}\right.$, $1 \mathrm{H}, 2-\mathrm{H}), 7.48-7.53(\mathrm{~m}, 2 \mathrm{H}, 5-\mathrm{H} / 6-\mathrm{H}), 8.13\left(\mathrm{dd},{ }^{3} J_{3,2}=8.3 \mathrm{~Hz},{ }^{4} J_{3,1}=1.6 \mathrm{~Hz}, 1 \mathrm{H}, 3-\mathrm{H}\right), 8.67(\mathrm{~m}, 1 \mathrm{H}, 7-$ H), 8.80 (dd, ${ }^{3} J_{1,2}=4.2 \mathrm{~Hz},{ }^{4} J_{1,3}=1.6 \mathrm{~Hz}, 1 \mathrm{H}, 1-\mathrm{H}$ ), 10.19 (bs, $1 \mathrm{H}, \mathrm{N}-\mathrm{H}$ ).

${ }^{13} \mathrm{C}-\mathrm{NMR}\left(100 \mathrm{MHz}, \mathrm{CDCl}_{3}\right): \delta=14.6$ (q, C-12), 25.2 (t, C-16), 27.1 (t, C-15), 51.2 (d, C-11), 55.5 (t, C-17), 65.3 (d, C-14), 66.6 (t, C-18), 116.6 (d, C-7), 121.7 (d, C-2), 122.0 (d, C-5), 127.1 (d, C-6), 127.8 (s, C-4), 133.9 (s, C-8), 136.2 (d, C-3), 138.4 (s, C-9), 148.5 (d, C-1), 168.3 (s, C-10), 175.2 (s, C-13).

Optical rotation: $\quad[\alpha]_{D}^{20}=-52.6\left[\mathrm{CHCl}_{3}, \mathrm{c}=1.00\right]$

HRMS (Cl) m/z: [M + H] $]^{+}$Calcd for $\mathrm{C}_{18} \mathrm{H}_{21} \mathrm{~N}_{4} \mathrm{O}_{2}$ 325.1659; Found 325.1654

\section{Benzyl ((S)-1-oxo-1-(((S)-1-oxo-1-(quinolin-8-ylamino)propan-2-yl)amino)propan-2-yl)carbamate (4)}

HOBt (644 mg, $4.20 \mathrm{mmol}, 1.05$ eq.) and EDC-HCl (806 mg, $4.20 \mathrm{mmol}, 1.05$ eq.) were added successively to a solution of Cbz-L-Ala-OH (894 mg, $4.00 \mathrm{mmol}, 1.0$ eq.) and 2-Amino- $N$-(quinolin-8yl)propenamide 1 ( $862 \mathrm{mg}, 4.00 \mathrm{mmol}, 1.0$ eq.) in $\mathrm{DCM}(40 \mathrm{~mL})$ at $0{ }^{\circ} \mathrm{C}$. The reaction mixture was warmed to room temperature overnight and before being hydrolyzed with aqueous $\mathrm{HCl}$ solution (1M). The mixture was extracted three times with DCM and the combined organic phases were washed successively with saturated $\mathrm{NaHCO}_{3}$ solution and dried over $\mathrm{Na}_{2} \mathrm{SO}_{4}$. After filtration, the solvent was evaporated and the crude product purified by column chromatography $\left(\mathrm{SiO}_{2}, \mathrm{DCM}: \mathrm{MeOH}\right.$ 100:0 $\rightarrow$ 95:5) to afford dipeptide 4 (1.28 g, $3.04 \mathrm{mmol}, 76 \%)$ as a white solid.

$\mathbf{R}_{\mathbf{f}}(\mathbf{4})=0.44\left(\mathrm{SiO}_{2}, \mathrm{DCM}: \mathrm{MeOH} 95: 5\right)$ 


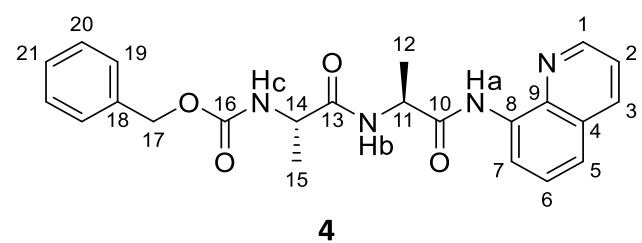

${ }^{1} \mathrm{H}-\mathrm{NMR}\left(400 \mathrm{MHz}, \mathrm{CDCl}_{3}\right): \delta=1.49\left(\mathrm{~d},{ }^{3} \mathrm{~J}_{15,14}=7.0 \mathrm{~Hz}, 3 \mathrm{H}, 15-\mathrm{H}\right), 1.55\left(\mathrm{~d},{ }^{3} \mathrm{~J}_{12,11}=6.7 \mathrm{~Hz}, 3 \mathrm{H}, 12-\mathrm{H}\right)$, $4.42\left(\mathrm{dq},{ }^{3} J_{14,15}=7.0 \mathrm{~Hz},{ }^{3} J_{14, \mathrm{NHc}}=6.9 \mathrm{~Hz}, 1 \mathrm{H}, 14-\mathrm{H}\right), 4.81\left(\mathrm{dq},{ }^{3} J_{11,12}=6.7 \mathrm{~Hz},{ }^{3} J_{11, \mathrm{NHb}}=5.1 \mathrm{~Hz}, 1 \mathrm{H}, 11-\mathrm{H}\right)$, $5.10\left(\mathrm{~d},{ }^{2} J_{17 \mathrm{a}, 17 \mathrm{~b}}=12.4 \mathrm{~Hz}, 1 \mathrm{H}, 17-\mathrm{H}_{\mathrm{a}}\right), 5.13\left(\mathrm{~d},{ }^{2} J_{17 \mathrm{~b}, 17 \mathrm{a}}=12.4 \mathrm{~Hz}, 1 \mathrm{H}, 17-\mathrm{H}_{\mathrm{b}}\right), 5.54\left(\mathrm{~d},{ }^{3} \mathrm{~J}_{\mathrm{NHc}, 14}=6.9 \mathrm{~Hz}, 1\right.$ $\left.\mathrm{H}, \mathrm{N}-\mathrm{H}_{\mathrm{c}}\right), 6.98\left(\mathrm{~d},{ }^{3} \mathrm{~J}_{\mathrm{NHb}, 11}=5.1 \mathrm{~Hz}, 1 \mathrm{H}, \mathrm{N}-\mathrm{H}_{\mathrm{b}}\right), 7.26-7.36(\mathrm{~m}, 5 \mathrm{H}, 19-\mathrm{H} / 20-\mathrm{H} / 21-\mathrm{H}), 7.44\left(\mathrm{dd},{ }^{3} \mathrm{~J}_{2,3}=8.3\right.$ $\left.\mathrm{Hz},{ }^{3} J_{2,1}=4.2 \mathrm{~Hz}, 1 \mathrm{H}, 2-\mathrm{H}\right), 7.49-7.53(\mathrm{~m}, 2 \mathrm{H}, 5-\mathrm{H} / 6-\mathrm{H}), 8.14\left(\mathrm{dd},{ }^{3} J_{3,2}=8.3 \mathrm{~Hz},{ }^{4} J_{3,1}=1.7 \mathrm{~Hz}, 1 \mathrm{H}, 3-\mathrm{H}\right)$, $8.70(\mathrm{~m}, 1 \mathrm{H}, 7-\mathrm{H}), 8.78\left(\mathrm{dd},{ }^{3} J_{1,2}=4.2 \mathrm{~Hz},{ }^{4} J_{1,3}=1.7 \mathrm{~Hz}, 1 \mathrm{H}, 1-\mathrm{H}\right), 10.19\left(\mathrm{bs}, 1 \mathrm{H}, \mathrm{N}-\mathrm{H}_{\mathrm{a}}\right)$.

${ }^{13} \mathrm{C}-N M R\left(100 \mathrm{MHz}, \mathrm{CDCl}_{3}\right.$ ): $\delta=18.8$ (q, C-12), 18.9 (q, C-15), 50.1 (d, C-11), 50.5 (d, C-14), 67.0 (t, C17), 116.6 (d, C-7), 121.7 (d, C-2), 122.0 (d, C-5), 127.2 (d, C-6), 127.9 (s, C-4), 128.0 (d, C-19), 128.1 (d, C-21), 128.5 (d, C-20), 133.9 (s, C-8), 136.2 (s, C-18), 136.3 (d, C-3), 138.4 (s, C-9), 148.3 (d, C-1), 155.9 (s, C-16), 170.3 (s, C-13), 172.2 (s, C-10).

Optical rotation: $\quad[\alpha]_{D}^{20}=-38.6\left[\mathrm{CHCl}_{3}, \mathrm{c}=1.00\right]$

Melting point: $\quad 184-186^{\circ} \mathrm{C}$

HRMS (Cl) m/z: [M + H] $]^{+}$Calcd for $\mathrm{C}_{23} \mathrm{H}_{25} \mathrm{~N}_{4} \mathrm{O}_{4} 421.1870$; Found 421.1856

\section{Benzyl (S)-5-methyl-4-oxo-3-((S)-1-oxo-1-(quinolin-8-ylamino)propan-2-yl)imidazoledine-1-carboxy- late (5)}

Hydrogenation:

$\mathrm{Pd} / \mathrm{C}$ (309 mg, dry, $10 \mathrm{wt} \% \mathrm{Pd}$ ) was added to a solution of dipeptide 4 (3.09 g, $7.35 \mathrm{mmol}, 1.0$ eq.) in $\mathrm{MeOH}(74 \mathrm{~mL})$ and the suspension was stirred under hydrogen atmosphere (1 atm) for 6 hours. Subsequently, the catalyst was removed by filtration through Celite and the solvent thereafter removed in vacuo to afford the free amine $(2.10 \mathrm{~g}, 7.32 \mathrm{mmol}, 100 \%)$ as a yellowish solid, which was used in the next step without further purification.

\section{Aminal formation:}

The previously prepared amine $(1.10 \mathrm{~g}, 3.84 \mathrm{mmol}, 1.0 \mathrm{eq}$.) was dissolved in formalin $(4.1 \mathrm{~mL}, 37 \mathrm{wt} \%$ in water) and the mixture was refluxed in an oil bath for 30 minutes. The resulting solution solution was then extracted three times with DCM and the combined organics were dried over $\mathrm{Na}_{2} \mathrm{SO}_{4}$. After solvent removal in vacuo, the crude product was purified by column chromatography $\left(\mathrm{C} 18, \mathrm{H}_{2} \mathrm{O}: \mathrm{MeCN}\right.$ 100:0 $\rightarrow 5: 95)$ to afford the aminal, which was directly used in the next step.

\section{Cbz-protection:}

$\mathrm{NaHCO}_{3}(645 \mathrm{mg}, 7.68 \mathrm{mmol}, 2.0$ eq.) and Cbz-chloride (603 $\mu \mathrm{L}, 721 \mathrm{mg}, 4.22 \mathrm{mmol}, 1.1$ eq.) were added successively to a solution of the previously prepared aminal in dioxane:water $(2: 1,16.7 \mathrm{~mL})$ and the reaction mixture was stirred at room temperature overnight. The resulting mixture was then 
diluted with aqueous $\mathrm{HCl}$ solution $(1 \mathrm{M})$ and extracted three times with ethyl acetate. Subsequently, the combined organic phases were dried over $\mathrm{Na}_{2} \mathrm{SO}_{4}$ and filtrated. After solvent removal in vacuo, the crude product was purified by column chromatography $\left(\mathrm{SiO}_{2}\right.$, PE:EtOAc 100:0 $\left.\rightarrow 50: 50\right)$ to afford carbamate 5 ( $1.12 \mathrm{~g}, 2.58 \mathrm{mmol}, 67 \%$ over 2 steps) as a colorless resin.

$\mathbf{R}_{\mathbf{f}}(\mathbf{5})=0.20\left(\mathrm{SiO}_{2}, \mathrm{PE}: \mathrm{EtOAC} 50: 50\right)$

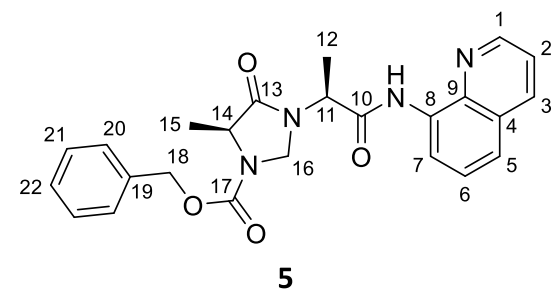

${ }^{1} \mathrm{H}-\mathrm{NMR}\left(500 \mathrm{MHz}\right.$, DMSO-d 6 , 373K): $\delta=1.44\left(\mathrm{~d},{ }^{3} J_{15,14}=6.6 \mathrm{~Hz}, 3 \mathrm{H}, 15-\mathrm{H}\right), 1.57\left(\mathrm{~d},{ }^{3} J_{12,11}=7.2 \mathrm{~Hz}, 3 \mathrm{H}\right.$, $12-\mathrm{H}), 4.25\left(\mathrm{dq},{ }^{3} J_{14,11}=6.6 \mathrm{~Hz},{ }^{4} J_{14,16 \mathrm{a}}=1.9 \mathrm{~Hz}, 1 \mathrm{H}, 14-\mathrm{H}\right), 4.93\left(\mathrm{dd},{ }^{2} J_{16 \mathrm{a}, 16 \mathrm{~b}}=6.3 \mathrm{~Hz},{ }^{4} J_{16 a, 14}=1.9 \mathrm{~Hz}, 1\right.$ $\left.\mathrm{H}, 16-\mathrm{H}_{\mathrm{a}}\right), 5.01\left(\mathrm{q},{ }^{3} J_{11,12}=7.2 \mathrm{~Hz}, 1 \mathrm{H}, 11-\mathrm{H}\right), 5.05\left(\mathrm{~d},{ }^{2} J_{16 \mathrm{~b}, 16 \mathrm{a}}=6.3 \mathrm{~Hz}, 1 \mathrm{H}, 16-\mathrm{H}_{\mathrm{b}}\right), 5.16\left(\mathrm{~d},{ }^{2} J_{18 a, 18 \mathrm{~b}}=12.6\right.$ $\left.\mathrm{Hz}, 1 \mathrm{H}, 18-\mathrm{H}_{\mathrm{a}}\right), 5.20\left(\mathrm{~d},{ }^{2} \mathrm{~J}_{18 \mathrm{~b}, 18 \mathrm{a}}=12.6 \mathrm{~Hz}, 18-\mathrm{H}_{\mathrm{b}}\right), 7.30-7.40(\mathrm{~m}, 5 \mathrm{H}, 20-\mathrm{H} / 21-\mathrm{H} / 22-\mathrm{H}), 7.58\left(\mathrm{dd},{ }^{3} \mathrm{~J}_{6,5}=\right.$ $8.5 \mathrm{~Hz}, 3_{6,7}^{3}=7.5 \mathrm{~Hz}, 1 \mathrm{H}, 6-\mathrm{H}$ ), $7.60\left(\mathrm{dd},{ }^{3} J_{2,3}=8.5 \mathrm{~Hz},{ }^{3} J_{2,1}=4.3 \mathrm{~Hz}, 1 \mathrm{H}, 2-\mathrm{H}\right), 7.68\left(\mathrm{dd},{ }^{3} 5_{5,6}=8.5 \mathrm{~Hz}\right.$, $\left.{ }^{4} J_{5,7}=1.3 \mathrm{~Hz}, 1 \mathrm{H}, 5-\mathrm{H}\right), 8.38\left(\mathrm{dd},{ }^{3} J_{3,2}=8.5 \mathrm{~Hz},{ }^{4} J_{3,1}=1.8 \mathrm{~Hz}, 1 \mathrm{H}, 3-\mathrm{H}\right.$ ), $8.54\left(\mathrm{dd},{ }^{3} J_{7,11}=7.5 \mathrm{~Hz},{ }^{4} J_{7,5}=1.3\right.$ $\mathrm{Hz}, 1 \mathrm{H}, 7-\mathrm{H}$ ), 8.85 (dd, $\left.{ }^{3} J_{1,2}=4.3 \mathrm{~Hz},{ }^{4} J_{1,3}=1.8 \mathrm{~Hz}, 1 \mathrm{H}, 1-\mathrm{H}\right), 10.22$ (bs, $1 \mathrm{H}, \mathrm{N}-\mathrm{H}$ ).

${ }^{13} \mathrm{C}-$ NMR (125 MHz, DMSO-d $\mathrm{d}_{6}$, 373K): $\delta=13.8$ (q, C-12), 16.2 (q, C-15), 50.6 (d, C-11), 53.8 (d, C-14), 58.9 (t, C-16), 66.1 (t, C-18), 116.4 (d, C-7), 121.5 (d, C-2), 121.8 (d, C-5), 126.2 (d, C-6), 127.1 (d, C-20), 127.4 (d, C-22), 127.4 (s, C-4), 127.8 (d, C-21), 133.4 (s, C-8), 135.9 (d, C-3), 135.9 (s, C-19), 138.0 (s, C9), 148.4 (d, C-1), 152.6 (s, C-17), 168.1 (s, C-13), 170.0 (s, C-10).

Optical rotation: $\quad[\alpha]_{D}^{20}=+62.6\left[\mathrm{CHCl}_{3}, \mathrm{c}=1.00\right]$

HRMS (Cl) m/z: [M + H] $]^{+}$Calcd for $\mathrm{C}_{24} \mathrm{H}_{25} \mathrm{~N}_{4} \mathrm{O}_{4}$ 433.1870; Found 433.1869

\section{Benzyl (S)-5-methyl-4-oxo-3-((S)-1-oxo-1-(quinolin-8-ylamino)-3-( $p$-tolyl)propan-2-yl)imidazolidine-} 1-carboxylate (6)

According to GP carbamate 5 (52.2 mg, $121 \mu \mathrm{mol}, 1.0$ eq.), 1-iodo-4-methylbenzene (52.7 mg, 242 $\mu \mathrm{mol}, 2.0$ eq.), silver carbonate ( $66.6 \mathrm{mg}, 242 \mu \mathrm{mol}, 2.0$ eq.), dibenzyl phosphate $(6.7 \mathrm{mg}, 24.2 \mu \mathrm{mol}$, $20 \mathrm{~mol} \%)$ and $\mathrm{Pd}(\mathrm{OAc})_{2}(2.7 \mathrm{mg}, 12.1 \mu \mathrm{mol}, 10 \mathrm{~mol} \%)$ were reacted at $60^{\circ} \mathrm{C}$ for 22 hours. The crude product was purified by column chromatography $\left(\mathrm{SiO}_{2}, \mathrm{PE}: \mathrm{EtOAc} 100: 0 \rightarrow 50: 50\right)$ and the product 6 (41.7 mg, $79.8 \mu \mathrm{mol}, 66 \%$ ) was obtained as a white lyophilisate.

$\mathbf{R}_{\mathbf{f}}(\mathbf{6})=0.36\left(\mathrm{SiO}_{2}, \mathrm{PE}: \mathrm{EtOAc} 70: 30\right)$ 


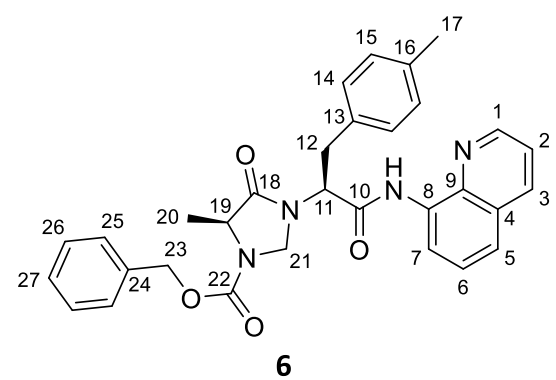

${ }^{1} \mathrm{H}-\mathrm{NMR}\left(500 \mathrm{MHz}\right.$, DMSO-d 6 , 373K): $\delta=1.15\left(\mathrm{~d},{ }^{3} \mathrm{~J}_{20,19}=6.6 \mathrm{~Hz}, 3 \mathrm{H}, 20-\mathrm{H}\right), 2.26(\mathrm{~s}, 3 \mathrm{H}, 17-\mathrm{H}), 3.20$ (dd, ${ }^{2} J_{12 a, 12 b}=14.4 \mathrm{~Hz},{ }^{3} J_{12 a, 11}=10.4 \mathrm{~Hz}, 1 \mathrm{H}, 12-\mathrm{H}_{\mathrm{a}}$ ), 3.41 (dd, ${ }^{2} J_{12 \mathrm{~b}, 12 \mathrm{a}}=14.4 \mathrm{~Hz},{ }^{3} J_{12 \mathrm{~b}, 11}=6.0 \mathrm{~Hz}, 1 \mathrm{H}, 12-$ $\left.\mathrm{H}_{\mathrm{b}}\right), 4.14\left(\mathrm{dq},{ }^{3} J_{19,20}=6.6 \mathrm{~Hz},{ }^{4} J_{19,21 \mathrm{a}}=1.6 \mathrm{~Hz}, 1 \mathrm{H}, 19-\mathrm{H}\right), 4.84\left(\mathrm{dd},{ }^{2} J_{21 \mathrm{a}, 21 \mathrm{~b}}=6.0 \mathrm{~Hz},{ }^{4} J_{21 \mathrm{a}, 19}=1.6 \mathrm{~Hz}, 1 \mathrm{H}\right.$, $\left.21-\mathrm{H}_{\mathrm{a}}\right), 4.99\left(\mathrm{~d},{ }^{2} J_{21 \mathrm{~b}, 21 \mathrm{a}}=6.0 \mathrm{~Hz}, 1 \mathrm{H}, 21-\mathrm{H}_{\mathrm{b}}\right), 5.12\left(\mathrm{~d},{ }^{2} J_{23 \mathrm{a}, 23 \mathrm{~b}}=12.6 \mathrm{~Hz}, 1 \mathrm{H}, 23-\mathrm{H}_{\mathrm{a}}\right), 5.16\left(\mathrm{~d},{ }^{2} J_{23 \mathrm{~b}, 23 \mathrm{a}}=\right.$ $\left.12.6 \mathrm{~Hz}, 1 \mathrm{H}, 23-\mathrm{H}_{\mathrm{b}}\right), 5.35\left(\mathrm{dd},{ }^{3} J_{11,12 \mathrm{a}}=10.4 \mathrm{~Hz},{ }^{3} J_{11,12 \mathrm{~b}}=6.0 \mathrm{~Hz}, 1 \mathrm{H}, 11-\mathrm{H}\right), 7.10\left(\mathrm{~d},{ }^{3} J_{14,15}=7.9 \mathrm{~Hz}, 2 \mathrm{H}\right.$, 14-H), $7.24\left(\mathrm{~d},{ }^{3} \mathrm{~J}_{15,14}=7.9 \mathrm{~Hz}, 2 \mathrm{H}, 15-\mathrm{H}\right), 7.30-7.38$ (sh, $\left.5 \mathrm{H}, 25-\mathrm{H} / 26-\mathrm{H} / 27-\mathrm{H}\right), 7.56-7.61$ (sh, $2 \mathrm{H}, 2-$ $\mathrm{H} / 6-\mathrm{H}), 7.69\left(\mathrm{dd}, 3_{5,6}=8.2 \mathrm{~Hz},{ }^{4} J_{5,7}=1.0 \mathrm{~Hz}, 1 \mathrm{H}, 5-\mathrm{H}\right), 8.37\left(\mathrm{dd},{ }^{3} J_{3,2}=8.2 \mathrm{~Hz},{ }^{4} J_{3,1}=1.6 \mathrm{~Hz}, 1 \mathrm{H}, 3-\mathrm{H}\right)$, $8.55\left(\mathrm{dd},{ }^{3} J_{7,6}=7.6 \mathrm{~Hz},{ }^{4} \mathrm{~J}_{7,5}=1.0 \mathrm{~Hz}, 1 \mathrm{H}, 7-\mathrm{H}\right), 8.84\left(\mathrm{dd},{ }^{3} J_{1,2}=4.1 \mathrm{~Hz},{ }^{4} J_{1,3}=1.6 \mathrm{~Hz}, 1 \mathrm{H}, 1-\mathrm{H}\right), 10.26(\mathrm{bs}$, $1 \mathrm{H}, \mathrm{N}-\mathrm{H})$.

${ }^{13}$ C-NMR (125 MHz, DMSO-d 6 , 373K): $\delta=16.1$ (q, C-20), 19.9 (q, C-17), 33.2 (t, C-12), 53.4 (d, C-19), 55.6 (d, C-11), 59.0 (t, C-21), 66.1 (t, C-23), 116.8 (d, C-7), 121.5 (d, C-2), 122.0 (d, C-5), 126.2 (d, C-6), 127.0 (d, C-25), 127.4 (d, C-27), 127.4 (s, C-4), 127.8 (d, C-26), 128.2 (d, C-15), 128.3 (d, C-14), 133.1 (s, C-13), 133.3 (s, C-8), 135.2 (s, C-16), 135.9 (d, C-3), 135.9 (s, C-24), 138.1 (s, C-9), 148.4 (d, C-1), 152.5 (s, C-22), 167.1 (s, C-10), 170.1 (s, C-18).

Optical rotation:

$$
[\alpha]_{D}^{20}=+48\left[\mathrm{CHCl}_{3}, \mathrm{c}=0.50\right]
$$

HRMS (Cl) m/z: [M + H] $]^{+}$Calcd for $\mathrm{C}_{31} \mathrm{H}_{31} \mathrm{~N}_{4} \mathrm{O}_{4}$ 523.2340; Found 523.2337

\section{(S)-2-((S)-4-Methyl-5-oxoimidazolidin-1-yl)-N-(quinolin-8-yl)propenamide (7)}

$\mathrm{Pd} / \mathrm{C}$ (10.0 mg, dry, $10 \mathrm{wt} \% \mathrm{Pd}$ ) was added to a solution of dipeptide 5 (97.0 mg, 224 umol, 1.0 eq.) in $\mathrm{MeOH}(1.1 \mathrm{~mL})$ and the suspension was stirred under hydrogen atmosphere (1 atm) for 4.5 hours. Subsequently, the catalyst was removed by filtration through Celite and the solvent thereafter removed in vacuo to afford amine 7 (66.8 mg, $224 \mu \mathrm{mol}, 100 \%$ ) as a colorless resin.

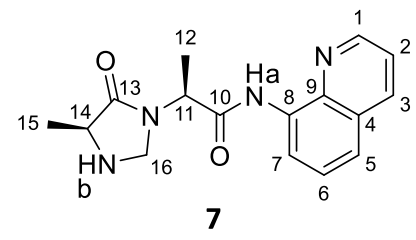

${ }^{1} \mathrm{H}-\mathrm{NMR}\left(400 \mathrm{MHz}, \mathrm{CDCl}_{3}\right): \delta=1.40\left(\mathrm{~d},{ }^{3} \mathrm{~J}_{15,14}=7.0 \mathrm{~Hz}, 3 \mathrm{H}, 15-\mathrm{H}\right), 1.58\left(\mathrm{~d},{ }^{3} \mathrm{~J}_{12,11}=7.2 \mathrm{~Hz}, 3 \mathrm{H}, 12-\mathrm{H}\right)$, 2.17 (bs, $1 \mathrm{H}, \mathrm{N}-\mathrm{H}_{\mathrm{b}}$ ), 3.65 (q, ${ }^{3} \mathrm{~J}_{14,15}=7.0 \mathrm{~Hz}, 1 \mathrm{H}, 14-\mathrm{H}$ ), 4.52 (d, ${ }^{2} \mathrm{~J}_{15,14}=7.8 \mathrm{~Hz}, 1 \mathrm{H}, 16-\mathrm{H}_{\mathrm{a}}$ ), 4.58 (d, $\left.{ }^{2} J_{15,14}=7.8 \mathrm{~Hz}, 1 \mathrm{H}, 16-\mathrm{H}_{\mathrm{b}}\right), 5.01\left(\mathrm{q},{ }^{3} J_{11,12}=7.2 \mathrm{~Hz}, 1 \mathrm{H}, 11-\mathrm{H}\right), 7.44\left(\mathrm{dd},{ }^{3} \mathrm{~J}_{2,3}=8.3 \mathrm{~Hz},{ }^{3} \mathrm{~J}_{2,1}=4.2 \mathrm{~Hz}, 1 \mathrm{H}\right.$, 2-H), $7.49-7.55$ (sh, $2 \mathrm{H}, 5-\mathrm{H} / 6-\mathrm{H}), 8.14\left(\mathrm{dd},{ }^{3} J_{3,2}=8.3 \mathrm{~Hz},{ }^{4} J_{3,1}=1.7 \mathrm{~Hz}, 1 \mathrm{H}, 3-\mathrm{H}\right), 8.68(\mathrm{~m}, 1 \mathrm{H}, 7-\mathrm{H})$, $8.81\left(\mathrm{dd},{ }^{3} J_{1,2}=4.2 \mathrm{~Hz},{ }^{4} J_{1,3}=1.6 \mathrm{~Hz}, 1 \mathrm{H}, 1-\mathrm{H}\right), 10.21\left(\mathrm{bs}, 1 \mathrm{H}, \mathrm{N}-\mathrm{H}_{\mathrm{a}}\right.$ ). 
${ }^{13} \mathrm{C}-N M R\left(100 \mathrm{MHz}, \mathrm{CDCl}_{3}\right.$ ): $\delta=14.5$ (q, C-12), 16.4 (q, C-15), 51.3 (d, C-11), 55.6 (d, C-14), 60.1 (t, C16), 116.5 (d, C-7), 121.6 (d, C-2), 122.0 (d, C-5), 127.1 (d, C-6), 127.8 (s, C-4), 133.8 (s, C-8), 136.2 (d, C-3), 138.4 (s, C-9), 148.5 (d, C-1), 168.5 (s, C-10), 176.3 (s, C-13).

Optical rotation: $\quad[\alpha]_{D}^{20}=-30\left[\mathrm{CHCl}_{3}, \mathrm{c}=1.00\right]$

HRMS (Cl) m/z: [M + H] $]^{+}$Calcd for $\mathrm{C}_{16} \mathrm{H}_{19} \mathrm{~N}_{4} \mathrm{O}_{2}$ 299.1503; Found 299.1493

\section{(S)-N-(Quinolin-8-yl)-2-((S)-2,2,4-trimethyl-5-oxoimidazolidin-1-yl)propenamide (8)}

\section{Hydrogenation:}

$\mathrm{Pd} / \mathrm{C}$ (309 mg, dry, $10 \mathrm{wt} \% \mathrm{Pd}$ ) was added to a solution of dipeptide 4 (3.09 g, $7.35 \mathrm{mmol}, 1.0$ eq.) in $\mathrm{MeOH}(74 \mathrm{~mL})$ and the suspension was stirred under hydrogen atmosphere (1 atm) for 6 hours. Subsequently, the catalyst was removed by filtration through Celite and the solvent thereafter removed in vacuo to afford the free amine $(2.10 \mathrm{~g}, 7.32 \mathrm{mmol}, 100 \%)$ as a yellowish solid, which was used in the next step without further purification.

\section{Aminal formation:}

A solution of the previously prepared free amine $(2.07 \mathrm{~g}, 7.23 \mathrm{mmol}, 1.0 \mathrm{eq}$.$) and p$-toluenesulfonic acid monohydrate $(13.8 \mathrm{mg}, 72.3 \mu \mathrm{mol}, 10 \mathrm{~mol} \%)$ in acetone $(15 \mathrm{~mL})$ was heated in an oil bath under reflux for 6 hours. Subsequently, the solvent was removed in vacuo and the residue diluted with $\mathrm{CHCl}_{3}$. The resulting solution was then washed with saturated $\mathrm{NaHCO}_{3}$ solution and dried over $\mathrm{Na}_{2} \mathrm{SO}_{4}$. After evaporating the solvent, the crude product was purified by column chromatography $\left(\mathrm{SiO}_{2}, \mathrm{DCM}: \mathrm{MeOH}\right.$ $100: 0 \rightarrow 95: 5)$ to afford aminal $8(2.01 \mathrm{~g}, 6.16 \mathrm{mmol}, 85 \%)$ as a yellowish solid.

$\mathbf{R}_{\mathbf{f}}(\mathbf{8})=0.28\left(\mathrm{SiO}_{2}, \mathrm{DCM}: \mathrm{MeOH} 95: 5\right)$

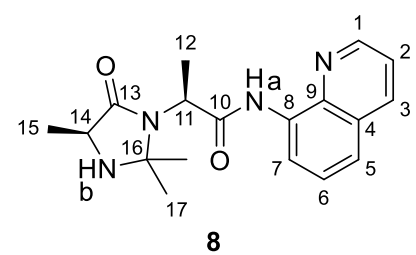

${ }^{1} \mathrm{H}-\mathrm{NMR}\left(400 \mathrm{MHz}, \mathrm{CDCl}_{3}\right): \delta=1.44(\mathrm{~s}, 3 \mathrm{H}, 17-\mathrm{H}), 1.49\left(\mathrm{~d},{ }^{3}{ }_{15,14}=6.7 \mathrm{~Hz}, 3 \mathrm{H}, 15-\mathrm{H}\right), 1.63(\mathrm{~s}, 3 \mathrm{H}, 17-$ $\left.\mathrm{H}^{\prime}\right), 1.82\left(\mathrm{~d},{ }^{3} \mathrm{~J}_{12,11}=7.2 \mathrm{~Hz}, 3 \mathrm{H}, 12-\mathrm{H}\right.$ ), $1.94\left(\mathrm{bs}, 1 \mathrm{H}, \mathrm{N}-\mathrm{H}_{\mathrm{b}}\right.$ ), $3.64\left(\mathrm{q},{ }^{3} \mathrm{~J}_{14,15}=6.7 \mathrm{~Hz}, 1 \mathrm{H}, 14-\mathrm{H}\right), 4.07$ (q, $\left.{ }^{3} J_{11,12}=7.2 \mathrm{~Hz}, 1 \mathrm{H}, 11-\mathrm{H}\right), 7.43\left(\mathrm{dd},{ }^{3} J_{2,3}=8.2 \mathrm{~Hz},{ }^{3} J_{2,1}=4.2 \mathrm{~Hz}, 1 \mathrm{H}, 2-\mathrm{H}\right), 7.48-7.55(\mathrm{~m}, 2 \mathrm{H}, 5-\mathrm{H} / 6-\mathrm{H})$, $8.14\left(\mathrm{dd},{ }^{3} J_{3,2}=8.2 \mathrm{~Hz},{ }^{4} J_{3,1}=1.7 \mathrm{~Hz}, 1 \mathrm{H}, 3-\mathrm{H}\right), 8.74\left(\mathrm{dd},{ }^{3} \mathrm{~J}_{7,6}=7.0 \mathrm{~Hz},{ }^{4} J_{7,5}=1.7 \mathrm{~Hz}, 1 \mathrm{H}, 7-\mathrm{H}\right), 8.77$ (dd, $\left.{ }^{3} J_{1,2}=4.2 \mathrm{~Hz},{ }^{4} J_{1,3}=1.7 \mathrm{~Hz}, 1 \mathrm{H}, 1-\mathrm{H}\right), 10.62\left(\mathrm{bs}, 1 \mathrm{H}, \mathrm{N}-\mathrm{H}_{\mathrm{a}}\right.$ ).

${ }^{13} \mathrm{C}-\mathrm{NMR}\left(100 \mathrm{MHz}, \mathrm{CDCl}_{3}\right.$ ): $\delta=14.5$ (q, C-12), 17.3 (q, C-15), 26.2 (q, C-17), 27.9 (q, C-17'), 53.6 (d, C11), 53.6 (d, C-14), 76.8 (s, C-16), 116.4 (d, C-7), 121.5 (d, C-2), 121.7 (d, C-5), 127.3 (d, C-6), 127.9 (s, C-4), 134.1 (s, C-8), 136.3 (d, C-3), 138.8 (s, C-9), 148.2 (d, C-1), 168.9 (s, C-10), 175.9 (s, C-13).

Optical rotation:

$$
[\alpha]_{D}^{20}=+104.6\left[\mathrm{CHCl}_{3}, \mathrm{c}=1.00\right]
$$

Melting point:

$$
140-143^{\circ} \mathrm{C} \text { (decomposition) }
$$

HRMS (Cl) m/z: [M + H] $]^{+}$Calcd for $\mathrm{C}_{28} \mathrm{H}_{23} \mathrm{~N}_{4} \mathrm{O}_{2}$ 327.1816; Found 327.1816 
Benzyl (S)-2,2,5-trimethyl-4-oxo-3-((S)-1-oxo-1-(quinolin-8-ylamino)propan-2-yl)imidazolidine-1carboxylate (9)

$\mathrm{NaHCO}_{3}$ (1.03 g, $12.3 \mathrm{mmol}, 2.0$ eq.) and Cbz-chloride ( $1.01 \mathrm{~mL}, 1.21 \mathrm{~g}, 6.74 \mathrm{mmol}, 1.1$ eq.) were added successively to a solution of aminal $8(2.00 \mathrm{~g}, 6.13 \mathrm{mmol}, 1.0$ eq.) in dioxane:water $(2: 1,26.6 \mathrm{~mL})$ and the reaction mixture was stirred at room temperature overnight. The resulting mixture was then diluted with aqueous $\mathrm{HCl}$ solution $(1 \mathrm{M})$ and extracted three times with ethyl acetate. Subsequently, the combined organics were dried over $\mathrm{Na}_{2} \mathrm{SO}_{4}$ and filtrated. After solvent removal in vacuo, the crude product was purified by column chromatography $\left(\mathrm{SiO}_{2}, \mathrm{PE}: \mathrm{EtOAc}\right.$ 100:0 $\rightarrow$ 50:50) to afford carbamate $9(2.37 \mathrm{~g}, 5.15 \mathrm{mmol}, 84 \%)$ as a colorless resin.

$\mathbf{R}_{\mathbf{f}}(\mathbf{9})=0.23\left(\mathrm{SiO}_{2}, \mathrm{PE}:\right.$ EtOAc 60:40)

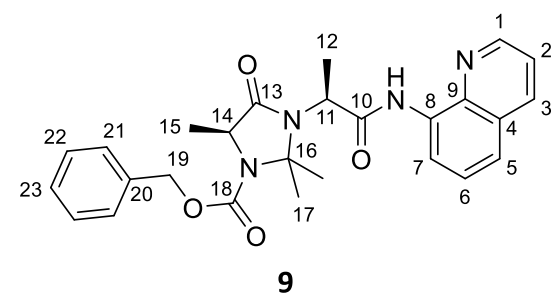

Major rotamer:

${ }^{1} \mathrm{H}-\mathrm{NMR}\left(400 \mathrm{MHz}, \mathrm{CDCl}_{3}\right): \delta=1.64\left(\mathrm{~d},{ }^{3} J_{15,14}=6.5 \mathrm{~Hz}, 3 \mathrm{H}, 15-\mathrm{H}\right), 1.81(\mathrm{~s}, 3 \mathrm{H}, 17-\mathrm{H}), 1.87\left(\mathrm{~d},{ }^{3} J_{12,11}=\right.$ $7.1 \mathrm{~Hz}, 3 \mathrm{H}, 12-\mathrm{H}), 1.96\left(\mathrm{~s}, 3 \mathrm{H}, 17-\mathrm{H}^{\prime}\right), 4.06\left(\mathrm{q},{ }^{3} \mathrm{~J}_{11,12}=7.1 \mathrm{~Hz}, 1 \mathrm{H}, 11-\mathrm{H}\right), 4.27\left(\mathrm{q},{ }^{3} J_{14,15}=6.5 \mathrm{~Hz}, 1 \mathrm{H}\right.$, $14-\mathrm{H}), 5.19\left(\mathrm{~d},{ }^{2} J_{19 a, 19 b}=12.4 \mathrm{~Hz}, 1 \mathrm{H}, 19-\mathrm{H}_{\mathrm{a}}\right), 5.23\left(\mathrm{~d},{ }^{2} \mathrm{~J}_{19 \mathrm{~b}, 19 \mathrm{a}}=12.4 \mathrm{~Hz},, 19-\mathrm{H}_{\mathrm{b}}\right), 7.32-7.41(\mathrm{~m}, 5 \mathrm{H}, 21-$ $\mathrm{H} / 22-\mathrm{H} / 23-\mathrm{H}$ ), $7.42\left(\mathrm{dd},{ }^{3} \mathrm{~J}_{2,3}=8.3 \mathrm{~Hz},{ }^{3} \mathrm{~J}_{2,1}=4.3 \mathrm{~Hz}, 1 \mathrm{H}, 2-\mathrm{H}\right), 7.48-7.55(\mathrm{~m}, 2 \mathrm{H}, 5-\mathrm{H} / 6-\mathrm{H}), 8.14$ (dd, $\left.{ }^{3} J_{3,2}=8.3 \mathrm{~Hz},{ }^{4} J_{3,1}=1.6 \mathrm{~Hz}, 1 \mathrm{H}, 3-\mathrm{H}\right), 8.73\left(\mathrm{dd},{ }^{3} J_{1,2}=4.3 \mathrm{~Hz},{ }^{4} J_{1,3}=1.6 \mathrm{~Hz}, 1 \mathrm{H}, 1-\mathrm{H}\right), 8.77\left(\mathrm{dd},{ }^{3} J_{7,6}=6.9\right.$ $\mathrm{Hz},{ }^{4} J_{7,5}=1.8 \mathrm{~Hz}$, , 7-H), 10.50 (bs, $1 \mathrm{H}, \mathrm{N}-\mathrm{H}$ ).

${ }^{13} \mathrm{C}-\mathrm{NMR}\left(100 \mathrm{MHz}, \mathrm{CDCl}_{3}\right.$ ): $\delta=15.4$ (q, C-12), 19.1 (q, C-15), 24.7 (q, C-17), 26.1 (q, C-17'), 52.8 (d, C11), 54.5 (d, C-14), 67.0 (t, C-19), 79.3 (s, C-16), 116.5 (d, C-7), 121.6 (d, C-2), 121.8 (d, C-5), 127.3 (d, C-6), 127.9 (s, C-4), 128.0 (d, C-21), 128.2 (d, C-23), 128.6 (d, C-22), 134.1 (s, C-8), 136.0 (s, C-20), 136.3 (d, C-3), 138.6 (s, C-9), 148.1 (d, C-1), 152.5 (s, C-18), 167.9 (s, C-10), 170.1 (s, C-13).

Minor rotamer (selected signals):

${ }^{1} \mathrm{H}-\mathrm{NMR}\left(400 \mathrm{MHz}, \mathrm{CDCl}_{3}\right): \delta=1.66-1.71(\mathrm{~m}, 2 \mathrm{H}, 15-\mathrm{H} / 17-\mathrm{H}), 1.87\left(\mathrm{~m}, 3 \mathrm{H}, 17-\mathrm{H}^{\prime}\right), 4.00(\mathrm{~m}, 1 \mathrm{H}, 11-$ $H), 4.34(\mathrm{~m}, 1 \mathrm{H}, 14-\mathrm{H}), 5.29(\mathrm{~m}, 2 \mathrm{H}, 19-\mathrm{H})$.

${ }^{13} \mathrm{C}-\mathrm{NMR}\left(100 \mathrm{MHz}, \mathrm{CDCl}_{3}\right.$ ): $\delta=17.8$ (q, C-12), 25.9 (q, C-17), 27.1 (q, C-17'), 53.1 (d, C-11), 55.0 (d, C14), 67.7 ( $t, C-19)$.

Optical rotation: $\quad[\alpha]_{D}^{20}=+78.4\left[\mathrm{CHCl}_{3}, \mathrm{c}=1.00\right]$

HRMS (Cl) m/z: [M + H] $]^{+}$Calcd for $\mathrm{C}_{28} \mathrm{H}_{29} \mathrm{~N}_{4} \mathrm{O}_{4} 461.2183$; Found 461.2178 
A solution of Boc-L-Ala-OH (5.00 g, $26.4 \mathrm{mmol}, 1.0$ eq.) in THF (160 mL) was cooled to $-20{ }^{\circ} \mathrm{C}$ before being treated with 4-methylmorpholine $(3.20 \mathrm{~mL}, 2.94 \mathrm{~g}, 29.1 \mathrm{mmol}, 1.1 \mathrm{eq}$.). Subsequently, isobutyl chloroformate (3.64 mL, $3.79 \mathrm{~g}, 27.7 \mathrm{mmol}, 1.05 \mathrm{eq}$.) was added dropwise and the resulting mixture stirred at $-20{ }^{\circ} \mathrm{C}$ for 20 minutes. Afterwards, 2-(methylthio)aniline (3.64 mL, $4.05 \mathrm{~g}, 29.1 \mathrm{mmol}, 1.1$ eq.) in THF ( $26 \mathrm{~mL}$ ) was added and the reaction mixture was warmed to room temperature overnight before it was diluted with EtOAc. The resulting mixture was washed successively with aqueous $\mathrm{HCl}$ solution (1M, 3x), saturated $\mathrm{NaHCO}_{3}$ solution (1x) and saturated $\mathrm{NaCl}$ solution (1x) followed by drying the organics over $\mathrm{Na}_{2} \mathrm{SO}_{4}$. After solvent removal in vacuo, the crude product was purified by column chromatography ( $\mathrm{SiO}_{2}, \mathrm{PE}:$ EtOAc 100:0 $\rightarrow$ 50:50) to obtain afford compound $\mathbf{1 1}$ (7.91 g, $25.5 \mathrm{mmol}, 96$ $\%)$ as a white solid.

$\mathbf{R}_{\mathbf{f}}(\mathbf{1 1})=0.46\left(\mathrm{SiO}_{2}, \mathrm{PE}: \mathrm{EtOAc} 60: 40\right)$

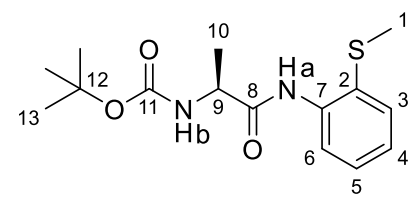

11

${ }^{1} \mathrm{H}-\mathrm{NMR}\left(400 \mathrm{MHz}, \mathrm{CDCl}_{3}\right): \delta=1.46(\mathrm{~s}, 9 \mathrm{H}, 13-\mathrm{H}), 1.48\left(\mathrm{~d},{ }^{3} \mathrm{~J}_{10,9}=7.3 \mathrm{~Hz}, 3 \mathrm{H}, 10-\mathrm{H}\right), 2.36(\mathrm{~s}, 3 \mathrm{H}, 1-\mathrm{H})$, $4.37(\mathrm{~m}, 1 \mathrm{H}, 9-\mathrm{H}), 5.09$ (bs, $1 \mathrm{H}, \mathrm{N}-\mathrm{H}_{\mathrm{b}}$ ), 7.06 (ddd, ${ }^{3} J_{4,3}=7.7 \mathrm{~Hz},{ }^{3} J_{4,5}=7.5 \mathrm{~Hz},{ }^{4} J_{4,6}=1.0 \mathrm{~Hz}, 1 \mathrm{H}, 4-\mathrm{H}$ ), 7.28 (ddd, ${ }^{3} J_{5,6}=8.1 \mathrm{~Hz},{ }^{3} J_{5,4}=7.5 \mathrm{~Hz},{ }^{4} J_{5,3}=1.2 \mathrm{~Hz}, 1 \mathrm{H}, 5-\mathrm{H}$ ), $7.47\left(\mathrm{dd},{ }^{3} J_{3,4}=7.7 \mathrm{~Hz},{ }^{4} J_{3,5}=1.2 \mathrm{~Hz}, 1 \mathrm{H}\right.$, $3-\mathrm{H}), 8.30\left(\mathrm{dd},{ }^{3} \mathrm{~J}_{6,5}=8.1 \mathrm{~Hz},{ }^{4} J_{6,4}=1.0 \mathrm{~Hz}, 1 \mathrm{H}, 6-\mathrm{H}\right), 8.98\left(\mathrm{bs}, 1 \mathrm{H}, \mathrm{N}-\mathrm{H}_{\mathrm{a}}\right)$.

${ }^{13} \mathrm{C}-\mathrm{NMR}\left(100 \mathrm{MHz}, \mathrm{CDCl}_{3}\right.$ ): $\delta=18.3$ (q, C-10), 18.9 (q, C-1), 28.3 (q, C-13), 51.2 (d, C-9), 80.3 (s, C-12), 120.5 (d, C-6), 124.5 (d, C-4), 125.7 (s, C-2), 128.8 (d, C-5), 132.9 (d, C-3), 138.0 (s, C-7), 155.4 (s, C-11), $170.9(\mathrm{~s}, \mathrm{C}-8)$.

Optical rotation: $\quad[\alpha]_{D}^{20}=-46.6\left[\mathrm{CHCl}_{3}, \mathrm{c}=1.00\right]$

Melting point: $\quad 106-107^{\circ} \mathrm{C}$

HRMS (Cl) m/z: [M + H] $]^{+}$Calcd for $\mathrm{C}_{15} \mathrm{H}_{23} \mathrm{~N}_{2} \mathrm{O}_{3} \mathrm{~S}$ 311.1424; Found 311.1416

tert-Butyl ((S)-1-(((S)-1-((2-(methylthio)phenyl)amino)-1-oxopropan-2-yl)amino)-1-oxopropan-2-yl)carbamate (12)

\section{Boc-deprotection:}

Acetyl chloride (18.2 mL, $20.1 \mathrm{~g}, 255 \mathrm{mmol}, 10$ eq.) was added dropwise to a solution of $\mathrm{MeOH}$ (11.4 $\mathrm{mL}, 9.00 \mathrm{~g}, 281 \mathrm{mmol}, 11$ eq.) in dioxane $(64 \mathrm{~mL})$ at $0{ }^{\circ} \mathrm{C}$. The resulting mixture was stirred at room temperature for 15 minutes before pouring it to compound 11 (7.93 g, $25.5 \mathrm{mmol}, 1.0$ eq.). After stirring the reaction mixture for 1.5 hours, the solvent was removed in vacuo to afford the amine hydrochloride, which was then used in the next step without further purification. 


\section{Peptide coupling:}

A solution of Boc-L-Ala-OH (4.82 g, $25.5 \mathrm{mmol}, 1.0$ eq.) in THF $(215 \mathrm{~mL})$ was cooled to $-20{ }^{\circ} \mathrm{C}$ and treated with 4-methylmorpholine $(3.10 \mathrm{~mL}, 2.85 \mathrm{~g}, 28.2 \mathrm{mmol}, 1.1$ eq.). Subsequently, isobutyl chloroformate ( $3.52 \mathrm{~mL}, 3.66 \mathrm{~g}, 26.8 \mathrm{mmol}, 1.05$ eq.) was added dropwise and the resulting mixture was stirred at $-20^{\circ} \mathrm{C}$ for 20 minutes. Afterwards, the previously prepared amine hydrochloride in THF $(40 \mathrm{~mL})$ and few drops of DCM as well as 4-methylmorpholine (3.07 mL, $2.82 \mathrm{~g}, 27.9 \mathrm{mmol}, 1.1 \mathrm{eq}$.) were added and the reaction mixture was warmed to room temperature overnight. The solvent was evaporated and the residue diluted with EtOAc. Afterwards, the resulting solution was washed successively with aqueous $\mathrm{HCl}$ solution( $\left(1 \mathrm{M}, 3 \mathrm{x}\right.$ ), saturated $\mathrm{NaHCO}_{3}$ solution $(1 \mathrm{x})$ and saturated $\mathrm{NaCl}$ solution (1x) followed by drying the organics over $\mathrm{Na}_{2} \mathrm{SO}_{4}$. After solvent removal in vacuo, the crude product was purified by column chromatography $\left(\mathrm{SiO}_{2}\right.$, $\mathrm{PE}: \mathrm{EtOAc}$ 100:0 $\left.\rightarrow 50: 50\right)$ to afford dipeptide $12(9.45 \mathrm{~g}, 24.8 \mathrm{mmol}, 97 \%)$ as a white solid.

$\mathbf{R}_{\mathbf{f}}(\mathbf{1 2})=0.27\left(\mathrm{SiO}_{2}, \mathrm{PE}: \mathrm{EtOAc} 50: 50\right)$

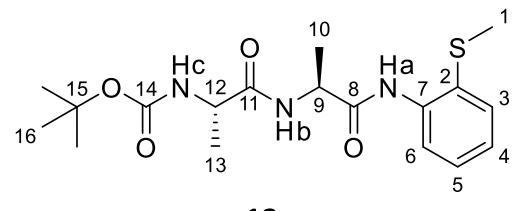

12

${ }^{1} \mathrm{H}-\mathrm{NMR}\left(400 \mathrm{MHz}, \mathrm{CDCl}_{3}\right): \delta=1.39\left(\mathrm{~d},{ }^{3} \mathrm{~J}_{13,12}=7.1 \mathrm{~Hz}, 3 \mathrm{H}, 13-\mathrm{H}\right), 1.43(\mathrm{~s}, 9 \mathrm{H}, 16-\mathrm{H}), 1.49\left(\mathrm{~d},{ }^{3} \mathrm{~J}_{10,9}=7.0\right.$ $\mathrm{Hz}, 3 \mathrm{H}, 10-\mathrm{H}), 2.35(\mathrm{~s}, 3 \mathrm{H}, 1-\mathrm{H}), 4.27(\mathrm{~m}, 1 \mathrm{H}, 12-\mathrm{H}), 4.66\left(\mathrm{dq},{ }^{3} \mathrm{~J}_{9, \mathrm{NHb}}=7.2 \mathrm{~Hz},{ }^{3} \mathrm{~J}_{9,10}=7.0 \mathrm{~Hz}, 1 \mathrm{H}, 9-\mathrm{H}\right)$, $5.13\left(\mathrm{~d},{ }^{3} J_{\mathrm{NHc}, 12}=7.5 \mathrm{~Hz}, 1 \mathrm{H}, \mathrm{N}-\mathrm{H}_{\mathrm{c}}\right), 7.06\left(\mathrm{~m}, 2 \mathrm{H}, 4-\mathrm{H} / \mathrm{N}-\mathrm{H}_{\mathrm{b}}\right), 7.26\left(\mathrm{ddd},{ }^{3} J_{5,6}=8.1 \mathrm{~Hz},{ }^{3} J_{5,4}=7.7 \mathrm{~Hz},{ }^{4} J_{5,3}=\right.$ $1.4 \mathrm{~Hz}, 1 \mathrm{H}, 5-\mathrm{H}), 7.45\left(\mathrm{dd},{ }^{3} J_{3,4}=7.8 \mathrm{~Hz},{ }^{4} J_{3,5}=1.3 \mathrm{~Hz}, 1 \mathrm{H}, 3-\mathrm{H}\right), 8.20\left(\mathrm{dd},{ }^{3} J_{6,5}=8.1 \mathrm{~Hz},{ }^{4} J_{6,4}=1.0 \mathrm{~Hz}, 1\right.$ $\mathrm{H}, 6-\mathrm{H}), 8.82$ (bs, $1 \mathrm{H}, \mathrm{N}-\mathrm{H}_{\mathrm{a}}$ ).

${ }^{13} \mathrm{C}-\mathrm{NMR}\left(100 \mathrm{MHz}, \mathrm{CDCl}_{3}\right.$ ): $\delta=18.0$ (q, C-10), 18.1 (q, C-13), 18.7 (q, C-1), 28.3 (q, C-16), 49.8 (d, C-9), 49.9 (d, C-12), 80.3 (s, C-15), 121.0 (d, C-6), 124.8 (d, C-4), 126.3 (s, C-2), 128.6 (d, C-5), 132.5 (d, C-3), 137.6 (s, C-7), 155.6 (s, C-14), 170.2 (s, C-8), 172.8 (s, C-11).

Optical rotation:

$[\alpha]_{D}^{20}=-62.8\left[\mathrm{CHCl}_{3}, \mathrm{c}=1.00\right]$

Melting point:

$84-85^{\circ} \mathrm{C}$

HRMS (Cl) m/z: $[\mathrm{M}+\mathrm{H}]^{+}$Calcd for $\mathrm{C}_{18} \mathrm{H}_{28} \mathrm{~N}_{3} \mathrm{O}_{4} \mathrm{~S}$ 382.1795; Found 382.1788

\section{Benzyl (S)-2,2,5-trimethyl-3-((S)-1-((2-(methylthio)phenyl)amino)-1-oxopropan-2-yl)-4-oxoimidazo- lidine-1-carboxylate (13)}

\section{Boc-deprotection:}

Acetyl chloride (17.2 mL, $19.0 \mathrm{~g}, 241 \mathrm{mmol}, 10 \mathrm{eq}$.) was added dropwise to a solution of $\mathrm{MeOH}(10.7$ $\mathrm{mL}, 8.51 \mathrm{~g}, 266 \mathrm{mmol}, 11$ eq.) in dioxane $(60 \mathrm{~mL})$ at $0{ }^{\circ} \mathrm{C}$. The resulting mixture was stirred at room temperature for 15 minutes before being poured to dipeptide $12(9.21 \mathrm{~g}, 24.1 \mathrm{mmol}, 1.0$ eq.). After stirring the reaction mixture for 1.5 hours the solvent was removed in vacuo to afford the amine hydrochloride, which was then treated with semi-saturated $\mathrm{Na}_{2} \mathrm{CO}_{3}$ solution. The aqueous phase was 
extracted three times with EtOAc and the combined organic phases were dried over $\mathrm{Na}_{2} \mathrm{SO}_{4}$. After solvent removal in vacuo, the free amine $(6.13 \mathrm{~g}, 21.8 \mathrm{mmol}, 90 \%)$ was obtained and used in the next step without further purification.

\section{Aminal formation:}

A solution of the previously prepared amine $(6.13 \mathrm{~g}, 21.8 \mathrm{mmol}, 1.0 \mathrm{eq}$.$) and p$-toluenesulfonic acid monohydrate ( $411 \mathrm{mg}, 2.16 \mathrm{mmol}, 10 \mathrm{~mol} \%$ ) in acetone $(43 \mathrm{~mL}$ ) was heated in an oil bath under reflux for 6 hours. Subsequently, the solvent was removed in vacuo and the residue diluted with $\mathrm{CHCl}_{3}$. The resulting solution was then washed with saturated $\mathrm{NaHCO}_{3}$ solution and dried over $\mathrm{Na}_{2} \mathrm{SO}_{4}$. After evaporating the solvent, the crude aminal was obtained and used in the next step without further purification.

\section{Cbz-protection:}

$\mathrm{NaHCO}_{3}$ (3.63 g, $43.2 \mathrm{mmol}, 2.0$ eq.) and Cbz-chloride ( $3.57 \mathrm{~mL}, 4.27 \mathrm{~g}, 23.8 \mathrm{mmol}, 1.1$ eq.) were added successively to a solution of the previously prepared aminal in dioxane:water $(2: 1,94 \mathrm{~mL})$ and the reaction mixture was stirred at room temperature for three days. The mixture was then diluted with aqueous $\mathrm{HCl}$ solution $(1 \mathrm{M})$ and extracted three times with ethyl acetate. Subsequently, the combined organic phases were dried over $\mathrm{Na}_{2} \mathrm{SO}_{4}$ and filtrated. After solvent removal in vacuo, the crude product was purified by column chromatography $\left(\mathrm{SiO}_{2}\right.$, PE:EtOAc 100:0 $\rightarrow$ 60:40) to afford carbamate 13 (8.32 $\mathrm{g}, 18.3 \mathrm{mmol}, 84 \%$ over 2 steps) as a colorless resin.

$\mathbf{R}_{\mathbf{f}}(\mathbf{1 3})=0.33\left(\mathrm{SiO}_{2}, \mathrm{PE}: \mathrm{EtOAc} 60: 40\right)$

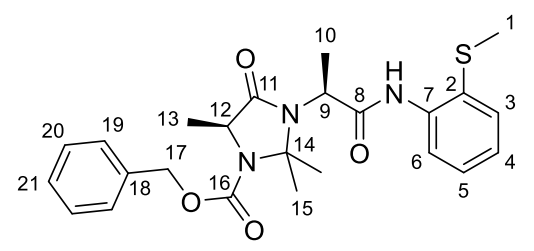

13

${ }^{1} \mathrm{H}-\mathrm{NMR}\left(500 \mathrm{MHz}\right.$, DMSO-d 6 , 373K): $\delta=1.44\left(\mathrm{~d},{ }^{3} \mathrm{~J}_{13,12}=6.6 \mathrm{~Hz}, 3 \mathrm{H}, 13-\mathrm{H}\right), 1.69\left(\mathrm{~d},{ }^{3} \mathrm{~J}_{10,9}=7.2 \mathrm{~Hz}, 3 \mathrm{H}\right.$, 10-H), $1.69(\mathrm{~s}, 3 \mathrm{H}, 15-\mathrm{H}), 1.77\left(\mathrm{~s}, 3 \mathrm{H}, 15-\mathrm{H}^{\prime}\right), 2.40(\mathrm{~s}, 3 \mathrm{H}, 1-\mathrm{H}), 4.18\left(\mathrm{q},{ }^{3} \mathrm{~J}_{12,13}=6.6 \mathrm{~Hz}, 1 \mathrm{H}, 12-\mathrm{H}\right), 4.32$ (q, $\left.{ }^{3} J_{9,10}=7.2 \mathrm{~Hz}, 1 \mathrm{H}, 9-\mathrm{H}\right), 5.16\left(\mathrm{~d},{ }^{2} J_{17 \mathrm{a}, 17 \mathrm{~b}}=12.2 \mathrm{~Hz}, 1 \mathrm{H}, 17-\mathrm{H}_{\mathrm{a}}\right), 5.21\left(\mathrm{~d},{ }^{2} J_{17 \mathrm{~b}, 17 \mathrm{a}}=12.2 \mathrm{~Hz}, 1 \mathrm{H}, 17-\mathrm{H}_{\mathrm{b}}\right.$ ), 7.15 (ddd, ${ }^{3} J_{4,3}=7.9 \mathrm{~Hz},{ }^{3} J_{4,5}=7.5 \mathrm{~Hz},{ }^{4} J_{4,6}=0.9 \mathrm{~Hz}, 1 \mathrm{H}, 4-\mathrm{H}$ ), 7.27 (ddd, ${ }^{3} J_{5,6}=7.9 \mathrm{~Hz},{ }^{3} J_{5,4}=7.5 \mathrm{~Hz},{ }^{4} J_{5,3}=$ $1.3 \mathrm{~Hz}, 1 \mathrm{H}, 5-\mathrm{H}), 7.32-7.43(\mathrm{~m}, 5 \mathrm{H}, 19-\mathrm{H} / 20-\mathrm{H} / 21-\mathrm{H}), 7.48$ (dd, ${ }^{3} J_{3,4}=7.9 \mathrm{~Hz},{ }^{4} J_{3,5}=1.3 \mathrm{~Hz}, 1 \mathrm{H}, 3-\mathrm{H}$ ), 7.99 (dd, $\left.{ }^{3} \sigma_{6,5}=7.9 \mathrm{~Hz},{ }^{4} \mathrm{~J}_{6,4}=0.9 \mathrm{~Hz}, 1 \mathrm{H}, 6-\mathrm{H}\right), 8.85(\mathrm{bs}, 1 \mathrm{H}, \mathrm{N}-\mathrm{H})$.

${ }^{13} \mathrm{C}-N M R$ (125 MHz, DMSO-d, 373K): $\delta=14.9$ (q, C-10), 17.0 (q, C-1), 18.0 (q, C-13), 23.8 (q, C-15), 25.6 (q, C-15'), 51.4 (d, C-9), 53.5 (d, C-12), 66.0 (t, C-17), 78.4 (s, C-14), 121.4 (d, C-6), 124.5 (d, C-4), 126.9 (d, C-5), 127.3 (d, C-19), 127.5 (d, C-21), 127.7 (s, C-2), 128.0 (d, C-20), 130.4 (d, C-3), 136.0 (s, C-18), 136.7 (s, C-7), 151.8 (s, C-16), 167.9 (s, C-8), 169.1 (s, C-11).

Optical rotation: $\quad[\alpha]_{D}^{20}=+51.0\left[\mathrm{CHCl}_{3}, \mathrm{c}=1.00\right]$

HRMS (Cl) m/z: [M + H] $]^{+}$Calcd for $\mathrm{C}_{24} \mathrm{H}_{30} \mathrm{~N}_{3} \mathrm{O}_{4} \mathrm{~S}$ 456.1952; Found 456.1940 


\section{Boc-protection:}

$\mathrm{Boc}_{2} \mathrm{O}$ (192 mg, $878 \mu \mathrm{mol}, 4.0$ eq.) and 4-dimethylaminopyridine (5.4 mg, $44.0 \mu \mathrm{mol}, 20 \mathrm{~mol} \%$ ) were added to a solution of carbamate $13(100 \mathrm{mg}, 220 \mu \mathrm{mol}, 1.0$ eq.) in MeCN (2.75 mL) and the reaction was stirred at room temperature overnight. The resulting dark solution was filtrated through a short silica column and the filtrate was concentrated to dryness in vacuo to afford the Boc-protected aminoquinoline-derivative, which was used in the next step without further purification.

\section{Saponification:}

The prepared derivative was dissolved in THF $(2.8 \mathrm{~mL})$ and cooled with an ice bath. Subsequently, lithium hydroxide monohydrate $(36.9 \mathrm{mg}, 878 \mu \mathrm{mol}, 4.0 \mathrm{eq}$.) in water $(1.4 \mathrm{~mL})$ and hydrogen peroxide solution ( $224 \mu \mathrm{L}, 2.20 \mathrm{mmol}, 10$ eq., $30 \mathrm{wt} \%$ ) were added at $0{ }^{\circ} \mathrm{C}$ and the reaction mixture was stirred at this temperature for 3 hours. In the next step, the mixture was diluted with aqueous sodium sulfite solution (1M), acidified with aqeous $\mathrm{HCl}$ solution (1M) and extracted three times with ethyl acetate. Afterwards, the combined organic phases were dried over $\mathrm{Na}_{2} \mathrm{SO}_{4}$ and filtrated. After solvent removal in vacuo, the crude product was purified by column chromatography $\left(\mathrm{SiO}_{2}\right.$, cyclohexane:EtOAc 100:0 $\rightarrow$ 50:50) to afford carbamate 14 (61.1 mg, $18.3 \mu \mathrm{mol}, 83 \%$ ) as a white lyophilisate.

$\mathbf{R}_{\mathbf{f}}(\mathbf{1 4})=0.23\left(\mathrm{SiO}_{2}, \mathrm{PE}:\right.$ EtOAc 50:50)

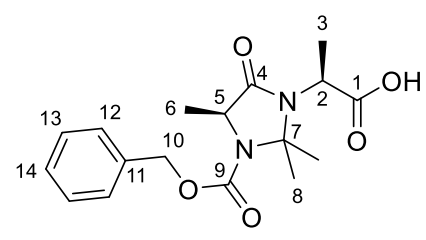

14

${ }^{1} \mathrm{H}-\mathrm{NMR}\left(\mathbf{5 0 0} \mathrm{MHz}\right.$, DMSO-d 6 , 373K): $\delta=1.34\left(\mathrm{~d},{ }^{3} \mathrm{~J}_{6,5}=6.6 \mathrm{~Hz}, 3 \mathrm{H}, 6-\mathrm{H}\right), 1.49\left(\mathrm{~d},{ }^{3} \mathrm{~J}_{3,2}=7.2 \mathrm{~Hz}, 3 \mathrm{H}, 3-\right.$ H), $1.63(\mathrm{~s}, 3 \mathrm{H}, 8-\mathrm{H}), 1.63\left(\mathrm{~s}, 3 \mathrm{H}, 8-\mathrm{H}^{\prime}\right), 4.08\left(\mathrm{q},{ }^{3} \mathrm{~J}_{2,3}=5.3 \mathrm{~Hz}, 1 \mathrm{H}, 2-\mathrm{H}\right), 4.09\left(\mathrm{q},{ }^{3} J_{5,6}=6.6 \mathrm{~Hz}, 1 \mathrm{H}, 5-\mathrm{H}\right)$, $5.14\left(\mathrm{~d},{ }^{2} J_{10 \mathrm{a}, 10 \mathrm{~b}}=12.6 \mathrm{~Hz}, 1 \mathrm{H}, 10-\mathrm{H}_{\mathrm{a}}\right), 5.19\left(\mathrm{~d},{ }^{2} J_{10 \mathrm{~b}, 10 \mathrm{a}}=12.6 \mathrm{~Hz}, 1 \mathrm{H}, 10-\mathrm{H}_{\mathrm{b}}\right), 7.33(\mathrm{~m}, 1 \mathrm{H}, 14-\mathrm{H}), 7.36-$ 7.41 (sh, $4 \mathrm{H}, 12-\mathrm{H} / 13-\mathrm{H} / 14-\mathrm{H}), 12.15$ (bs, $1 \mathrm{H}, \mathrm{COO}-\mathrm{H})$.

${ }^{13} \mathrm{C}-N M R\left(125 \mathrm{MHz}\right.$, DMSO-d $\left.\mathrm{d}_{6}, 373 \mathrm{~K}\right): \delta=15.1$ (q, C-3), 17.5 (q, C-6), 24.4 (q, C-8/C-8'), 24.7 (q, C-8/C8'), 48.4 (d, C-2), 53.2 (d, C-5), 65.8 (t, C-10), 77.9 (s, C-7), 127.1 (d, C-12), 127.4 (d, C-14), 127.9 (d, C13), 136.0 (s, C-11), 151.6 (s, C-9), 167.7 (s, C-4), 170.6 (s, C-1).

Optical rotation: $\quad[\alpha]_{D}^{20}=+14\left[\mathrm{CHCl}_{3}, \mathrm{c}=0.50\right]$

HRMS (Cl) m/z: [M + H] $]^{+}$Calcd for $\mathrm{C}_{17} \mathrm{H}_{23} \mathrm{~N}_{2} \mathrm{O}_{5}$ 335.1601; Found 335.1601

Benzyl (S)-2,2,5-trimethyl-3-((S)-1-((2-(methylthio)phenyl)amino)-1-oxo-3-(p-tolyl)propan-2-yl)-4oxoimidazolidine-1-carboxylate (15a)

According to GP carbamate 13 (214 mg, $470 \mu \mathrm{mol}, 1.0$ eq.), 4-iodotoluene (205 mg, $939 \mu \mathrm{mol}, 2.0$ eq.), silver carbonate ( $259 \mathrm{mg}, 939 \mu \mathrm{mol}, 2.0$ eq.), dibenzyl phosphate $(26.1 \mathrm{mg}, 93.8 \mu \mathrm{mol}, 20 \mathrm{~mol} \%$ ) and $\mathrm{Pd}(\mathrm{OAc})_{2}(10.6 \mathrm{mg}, 47.2 \mu \mathrm{mol}, 10 \mathrm{~mol} \%)$ were reacted at $60^{\circ} \mathrm{C}$ for 3 days. The crude product was 
purified by column chromatography $\left(\mathrm{SiO}_{2}\right.$, PE:EtOAc 100:0 $\rightarrow$ 80:20) and the product 15a (175 mg, 321 $\mu \mathrm{mol}, 68 \%)$ was obtained as a white lyophilisate.

$\mathbf{R}_{\mathbf{f}}(\mathbf{1 5 a})=0.20\left(\mathrm{SiO}_{2}\right.$, PE:EtOAc 80:20)

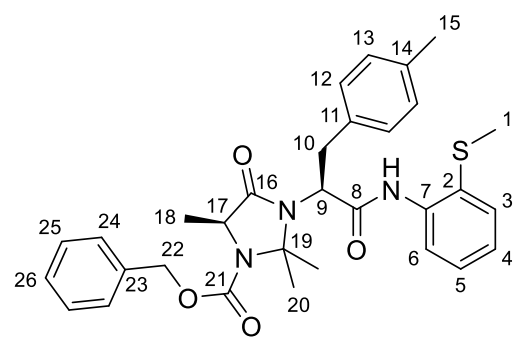

$15 a$

${ }^{1} \mathrm{H}-\mathrm{NMR}\left(500 \mathrm{MHz}\right.$, DMSO-d 6 , 373K): $\delta=1.04(\mathrm{~s}, 3 \mathrm{H}, 20-\mathrm{H}), 1.45$ (d, ${ }^{3} \mathrm{~J}_{18,17}=6.6 \mathrm{~Hz}, 3 \mathrm{H}, 18-\mathrm{H}$ ), 1.60 (s, $\left.3 \mathrm{H}, 20-\mathrm{H}^{\prime}\right), 2.28(\mathrm{~s}, 3 \mathrm{H}, 15-\mathrm{H}), 2.42(\mathrm{~s}, 3 \mathrm{H}, 1-\mathrm{H}), 3.49\left(\mathrm{dd},{ }^{2} J_{10 \mathrm{a}, 10 \mathrm{~b}}=13.5 \mathrm{~Hz},{ }^{3} J_{10 \mathrm{a}, 9}=5.7 \mathrm{~Hz}, 1 \mathrm{H}, 10-\mathrm{H}_{\mathrm{a}}\right)$, $3.76\left(\mathrm{dd},{ }^{2} J_{10 \mathrm{~b}, 10 \mathrm{a}}=13.5 \mathrm{~Hz},{ }^{3} J_{10 \mathrm{~b}, 9}=9.7 \mathrm{~Hz}, 1 \mathrm{H}, 10-\mathrm{H}_{\mathrm{b}}\right), 4.12\left(\mathrm{q},{ }^{3} J_{17,18}=6.6 \mathrm{~Hz}, 1 \mathrm{H}, 17-\mathrm{H}\right), 4.21$ (dd, $\left.{ }^{3} J_{g, 10 b}=9.7 \mathrm{~Hz},{ }^{3} J_{g, 10 a}=5.7 \mathrm{~Hz}, 1 \mathrm{H}, 9-\mathrm{H}\right), 5.10\left(\mathrm{~d},{ }^{2} J_{22 a, 22 b}=12.6 \mathrm{~Hz}, 1 \mathrm{H}, 22-\mathrm{H}_{\mathrm{a}}\right), 5.15\left(\mathrm{~d},{ }^{2} \mathrm{~J}_{22 \mathrm{~b}, 22 \mathrm{a}}=12.6 \mathrm{~Hz}\right.$, $1 \mathrm{H}, 22-\mathrm{H}_{\mathrm{b}}$ ), $7.12\left(\mathrm{~d},{ }^{3} \mathrm{~J}_{12,13}=7.9 \mathrm{~Hz}, 2 \mathrm{H}, 12-\mathrm{H}\right.$ ), 7.19 (ddd, ${ }^{3} J_{4,3}=7.9 \mathrm{~Hz},{ }^{3} J_{4,5}=7.5 \mathrm{~Hz},{ }^{4} J_{4,6}=1.6 \mathrm{~Hz}, 1 \mathrm{H}$, 4-H), 7.22 (d, $\left.{ }^{3} J_{13,12}=7.9 \mathrm{~Hz}, 2 \mathrm{H}, 13-\mathrm{H}\right), 7.24$ (ddd, ${ }^{3} J_{5,6}=7.9 \mathrm{~Hz},{ }^{3} J_{5,4}=7.5 \mathrm{~Hz},{ }^{4} J_{5,3}=1.6 \mathrm{~Hz}, 1 \mathrm{H}, 5-\mathrm{H}$ ), $7.31-7.40(\mathrm{~m}, 5 \mathrm{H}, 24-\mathrm{H} / 25-\mathrm{H} / 26-\mathrm{H}), 7.45\left(\mathrm{dd},{ }^{3} J_{3,4}=7.9 \mathrm{~Hz},{ }^{4} J_{3,5}=1.6 \mathrm{~Hz}, 1 \mathrm{H}, 3-\mathrm{H}\right), 7.75\left(\mathrm{dd},{ }^{3} J_{6,5}=7.9\right.$ $\left.\mathrm{Hz},{ }^{4} J_{6,4}=1.6 \mathrm{~Hz}, 1 \mathrm{H}, 6-\mathrm{H}\right), 9.25$ (bs, $\left.1 \mathrm{H}, \mathrm{N}-\mathrm{H}\right)$.

${ }^{13}$ C-NMR (125 MHz, DMSO-d, , 373K): $\delta=16.2$ (q, C-1), 18.0 (q, C-18), 20.0 (q, C-15), 22.9 (q, C-20), 25.5 (q, C-20), 33.9 (t, C-10), 53.5 (d, C-17), 59.5 (d, C-9), 66.0 (t, C-22), 78.6 (s, C-19), 123.2 (d, C-6), 125.2 (d, C-4), 126.1 (d, C-5), 127.2 (d, C-24), 127.4 (d, C-26), 127.9 (d, C-25), 128.3 (d, C-13), 128.9 (d, C-12), 129.1 (d, C-3), 129.9 (s, C-2), 134.0 (s, C-14), 135.2 (s, C-11), 135.8 (s, C-23), 135.9 (s, C-7), 151.6 (s, C21), 167.5 (s, C-8), 170.0 (s, C-16).

Optical rotation:

$$
[\alpha]_{D}^{20}=-24.2\left[\mathrm{CHCl}_{3}, \mathrm{c}=1.00\right]
$$

HRMS (Cl) m/z: [M + H] $]^{+}$Calcd for $\mathrm{C}_{31} \mathrm{H}_{36} \mathrm{~N}_{3} \mathrm{O}_{4} \mathrm{~S}$ 546.2421; Found 546.2428

\section{Benzyl (S)-2,2,5-trimethyl-3-((S)-1-((2-(methylthio)phenyl)amino)-1-oxo-3-phenylpropan-2-yl)-4- oxoimidazolidine-1-carboxylate (15b)}

According to GP carbamate 13 (65.3 mg, $143 \mu \mathrm{mol}, 1.0$ eq.), iodobenzene ( $32.1 \mu \mathrm{L}, 58.5 \mathrm{mg}, 287 \mu \mathrm{mol}$, 2.0 eq.), silver carbonate (79.0 mg, $287 \mu \mathrm{mol}, 2.0$ eq.), dibenzyl phosphate $(8.1 \mathrm{mg}, 29.0 \mu \mathrm{mol}, 20$ mol\%) and $\mathrm{Pd}(\mathrm{OAc})_{2}(3.3 \mathrm{mg}, 14.5 \mu \mathrm{mol}, 10 \mathrm{~mol} \%)$ were reacted at $60^{\circ} \mathrm{C}$ for 2 days. The crude product was purified by column chromatography $\left(\mathrm{SiO}_{2}, \mathrm{PE}:\right.$ EtOAc 100:0 $\rightarrow$ 80:20) and the product 15b (51.6 $\mathrm{mg}, 97.1 \mu \mathrm{mol}, 67 \%)$ was obtained as a colorless resin.

$\mathbf{R}_{\mathbf{f}}(\mathbf{1 5 b})=0.34\left(\mathrm{SiO}_{2}, \mathrm{PE}:\right.$ EtOAc 70:30) 


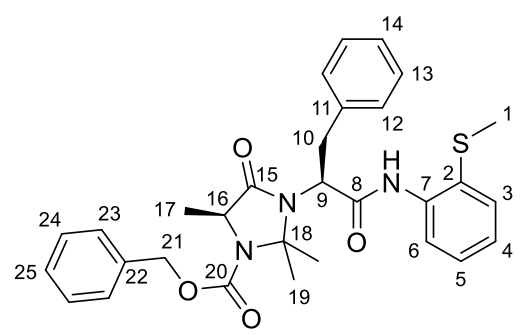

$15 b$

${ }^{1} \mathrm{H}-\mathrm{NMR}\left(500 \mathrm{MHz}\right.$, DMSO-d 6 , 373K): $\delta=1.01(\mathrm{~s}, 3 \mathrm{H}, 19-\mathrm{H}), 1.45$ (d, $\left.{ }^{3} \mathrm{~J}_{17,16}=6.6 \mathrm{~Hz}, 3 \mathrm{H}, 17-\mathrm{H}\right), 1.61$ (s, $\left.3 \mathrm{H}, 19-\mathrm{H}^{\prime}\right), 2.43(\mathrm{~s}, 3 \mathrm{H}, 1-\mathrm{H}), 3.53\left(\mathrm{dd},{ }^{2} J_{10 \mathrm{a}, 10 \mathrm{~b}}=13.5 \mathrm{~Hz},{ }^{3} J_{10 \mathrm{a}, 9}=5.7 \mathrm{~Hz}, 1 \mathrm{H}, 10-\mathrm{H}_{\mathrm{a}}\right), 3.82\left(\mathrm{dd},{ }^{2} J_{10 \mathrm{~b}, 10 \mathrm{a}}=\right.$ $13.5 \mathrm{~Hz},{ }^{3} \mathrm{~J}_{10 \mathrm{~b}, 9}=10.1 \mathrm{~Hz}, 1 \mathrm{H}, 10-\mathrm{H}_{\mathrm{b}}$ ), $4.12\left(\mathrm{q},{ }^{3} \mathrm{~J}_{16,17}=6.6 \mathrm{~Hz}, 1 \mathrm{H}, 16-\mathrm{H}\right), 4.25$ (dd, ${ }^{3} \mathrm{~J}_{9,10 \mathrm{~b}}=10.1 \mathrm{~Hz},{ }^{3} \mathrm{~g}_{9,10 \mathrm{a}}=$ $5.7 \mathrm{~Hz}, 1 \mathrm{H}, 9-\mathrm{H}), 5.10\left(\mathrm{~d},{ }^{2} J_{21 \mathrm{a}, 21 \mathrm{~b}}=12.6 \mathrm{~Hz}, 1 \mathrm{H}, 21-\mathrm{H}_{\mathrm{a}}\right), 5.15\left(\mathrm{~d},{ }^{2} \mathrm{~J}_{21 \mathrm{~b}, 21 \mathrm{a}}=12.6 \mathrm{~Hz}, 1 \mathrm{H}, 21-\mathrm{H}_{\mathrm{b}}\right), 7.19$ (ddd, $\left.{ }^{3} J_{4,3}=7.9 \mathrm{~Hz},{ }^{3} J_{4,5}=7.5 \mathrm{~Hz},{ }^{4} J_{4,6}=1.6 \mathrm{~Hz}, 1 \mathrm{H}, 4-\mathrm{H}\right), 7.22-7.26(\mathrm{~m}, 2 \mathrm{H}, 5-\mathrm{H} / 14-\mathrm{H}), 7.29-7.40(\mathrm{~m}, 9 \mathrm{H}$, $12-\mathrm{H} / 13-\mathrm{H} / 23-\mathrm{H} / 24-\mathrm{H} / 25-\mathrm{H}), 7.45\left(\mathrm{dd},{ }^{3} J_{3,4}=7.9 \mathrm{~Hz},{ }^{4} J_{3,5}=1.6 \mathrm{~Hz}, 1 \mathrm{H}, 3-\mathrm{H}\right), 7.75$ (dd, ${ }^{3} J_{6,5}=7.9 \mathrm{~Hz}$, $\left.{ }^{4} J_{6,4}=1.6 \mathrm{~Hz}, 1 \mathrm{H}, 6-\mathrm{H}\right), 9.26$ (bs, $\left.1 \mathrm{H}, \mathrm{N}-\mathrm{H}\right)$.

${ }^{13}$ C-NMR (125 MHz, DMSO-d 6 , 373 K): $\delta=16.2$ (q, C-1), 17.9 (q, C-17), 22.8 (q, C-19), 25.4 (q, C-19'), 34.3 (t, C-10), 53.5 (d, C-16), 59.3 (d, C-9), 66.0 (t, C-21), 78.5 (s, C-18), 123.3 (d, C-6), 125.2 (d, C-4), 126.1 (d, C-5), 127.2/127.4/127.7/127.9 (d, C-13/C-14/C-23/C-24/C-25), 129.0 (d, C-12), 129.1 (d, C-3), 130.0 (s, C-2), 135.8 (s, C-22), 135.9 (s, C-7), 137.2 (s, C-11), 151.6 (s, C-20), 167.5 (s, C-8), 170.0 (s, C$15)$.

Optical rotation: $\quad[\alpha]_{D}^{20}=-19.7\left[\mathrm{CHCl}_{3}, \mathrm{c}=1.00\right]$

HRMS (Cl) m/z: $[\mathrm{M}+\mathrm{H}]^{+}$Calcd for $\mathrm{C}_{30} \mathrm{H}_{34} \mathrm{~N}_{3} \mathrm{O}_{4} \mathrm{~S}$ 532.2265; Found 532.2256

Benzyl (S)-3-((S)-3-(4-methoxyphenyl)-1-((2-(methylthio)phenyl)amino)-1-oxopropan-2-yl)-2,2,5-trimethyl-4-oxoimidazolidine-1-carboxylate (15c)

According to GP carbamate 13 (65.6 mg, $144 \mu \mathrm{mol}, 1.0$ eq.), 4-iodoanisole (67.4 mg, $288 \mu \mathrm{mol}, 2.0$ eq.), silver carbonate $(79.0 \mathrm{mg}, 288 \mu \mathrm{mol}, 2.0$ eq.), dibenzyl phosphate $(8.0 \mathrm{mg}, 28.8 \mu \mathrm{mol}, 20 \mathrm{~mol} \%)$ and $\mathrm{Pd}(\mathrm{OAc})_{2}(3.2 \mathrm{mg}, 14.3 \mu \mathrm{mol}, 10 \mathrm{~mol} \%)$ were reacted at $60^{\circ} \mathrm{C}$ for 2 days. The crude product was purified successively by normal phase column chromatography $\left(\mathrm{SiO}_{2}, \mathrm{PE}: \mathrm{EtOAc}\right.$ 100:0 $\rightarrow$ 80:20) and reverse phase column chromatography $\left(\mathrm{C} 18, \mathrm{H}_{2} \mathrm{O}: \mathrm{MeCN} 100: 0 \rightarrow 0: 100\right)$ and the product $15 \mathrm{c}(53.1 \mathrm{mg}, 94.5$ $\mu \mathrm{mol}, 66 \%)$ was obtained as a white lyophilisate.

$\mathbf{R}_{\mathbf{f}}(\mathbf{1 5 c})=0.24\left(\mathrm{SiO}_{2}, \mathrm{PE}:\right.$ EtOAc 70:30)

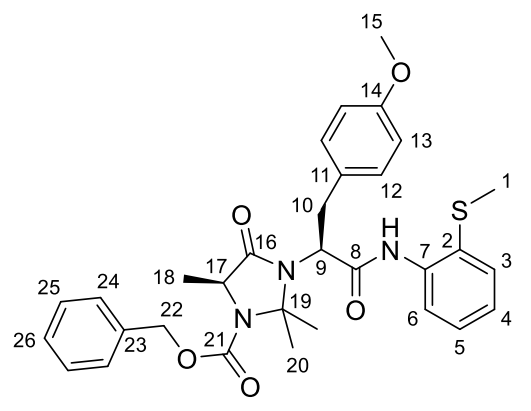

$15 c$ 


\section{Major rotamer:}

${ }^{1} \mathrm{H}-\mathrm{NMR}\left(400 \mathrm{MHz}, \mathrm{CDCl}_{3}\right): \delta=0.98(\mathrm{~s}, 3 \mathrm{H}, 20-\mathrm{H}), 1.56(\mathrm{~m}, 3 \mathrm{H}, 18-\mathrm{H}), 1.66\left(\mathrm{~s}, 3 \mathrm{H}, 20-\mathrm{H}^{\prime}\right), 2.42(\mathrm{~s}, 3 \mathrm{H}$, 1-H), $3.37\left(\mathrm{~m}, 1 \mathrm{H}, 10-\mathrm{H}_{\mathrm{a}}\right), 3.78(\mathrm{~s}, 3 \mathrm{H}, 15-\mathrm{H}), 3.85-4.05\left(\mathrm{~m}, 2 \mathrm{H}, 10-\mathrm{H}_{\mathrm{b}} / 9-\mathrm{H}\right), 4.19(\mathrm{~m}, 1 \mathrm{H}, 17-\mathrm{H}), 5.07$ $\left(d,{ }^{2} J_{22 a, 22 b}=12.2 \mathrm{~Hz}, 1 \mathrm{H}, 22-\mathrm{H}_{\mathrm{a}}\right), 5.16\left(\mathrm{~d},{ }^{2} J_{22 \mathrm{~b}, 22 \mathrm{a}}=12.2 \mathrm{~Hz}, 1 \mathrm{H}, 22-\mathrm{H}_{\mathrm{b}}\right), 6.83\left(\mathrm{~d},{ }^{3} \mathrm{~J}_{12,13}=8.2 \mathrm{~Hz}, 2 \mathrm{H}, 12-\right.$ H), $7.13\left(\mathrm{dd},{ }^{3} \mathrm{~J}_{4,3}=7.8 \mathrm{~Hz},{ }^{3} \mathrm{~J}_{4,5}=7.5 \mathrm{~Hz}, 1 \mathrm{H}, 4-\mathrm{H}\right), 7.18\left(\mathrm{~d},{ }^{3} J_{13,12}=8.2 \mathrm{~Hz}, 2 \mathrm{H}, 13-\mathrm{H}\right), 7.26\left(\mathrm{ddd},{ }^{3} J_{5,6}=\right.$ $\left.8.1 \mathrm{~Hz},{ }^{3} J_{5,4}=7.5 \mathrm{~Hz} .{ }^{4} J_{5,3}=0.9 \mathrm{~Hz}, 1 \mathrm{H}, 5-\mathrm{H}\right), 7.30-7.39(\mathrm{~m}, 5 \mathrm{H}, 24-\mathrm{H} / 25-\mathrm{H} / 26-\mathrm{H}), 7.41$ (dd, ${ }^{3} 3_{3,4}=7.8$ $\left.\mathrm{Hz},{ }^{4} J_{3,5}=0.9 \mathrm{~Hz}, 1 \mathrm{H}, 3-\mathrm{H}\right), 8.03\left(\mathrm{~d},{ }^{3} \mathrm{~J}_{6,5}=8.1 \mathrm{~Hz}, 1 \mathrm{H}, 6-\mathrm{H}\right), 9.60$ (bs, $\left.1 \mathrm{H}, \mathrm{N}-\mathrm{H}\right)$.

${ }^{13} \mathrm{C}-\mathrm{NMR}\left(100 \mathrm{MHz}, \mathrm{CDCl}_{3}\right.$ ): $\delta=17.8$ (q, C-1), 19.4 (q, C-18), 22.8 (q, C-20), 26.5 (q, C-20'), 33.9 (t, C10), 54.4 (d, C-17), 55.2 (q, C-15), 63.0 (d, C-9), 67.0 (t, C-22), 80.0 (s, C-19), 114.0 (d, C-12), 122.8 (d, C-6), 125.5 (d, C-4), 127.5 (d, C-5), 128.1 (d, C-24), 128.3 (d, C-26), 128.6 (d, C-25), 128.8 (s, C-2), 128.8 (s, C-11), 130.5 (d, C-13), 130.8 (d, C-3), 135.8 (s, C-23), 136.9 (s, C-7), 152.3 (s, C-21), 158.7 (s, C-14), 169.3 (s, C-8), 171.8 (s, C-16).

\section{Minor rotamer (selected signals):}

${ }^{1} \mathrm{H}-\mathrm{NMR}\left(400 \mathrm{MHz}, \mathrm{CDCl}_{3}\right): \delta=0.81(\mathrm{~s}, 3 \mathrm{H}, 20-\mathrm{H}), 4.26(\mathrm{~m}, 1 \mathrm{H}, 17-\mathrm{H})$.

${ }^{13} \mathrm{C}-\mathrm{NMR}\left(100 \mathrm{MHz}, \mathrm{CDCl}_{3}\right): \delta=23.9$ (q, C-20), 27.4 (q, C-20'), 63.3 (d, C-9), 67.5 (t, C-22).

Optical rotation: $\quad[\alpha]_{D}^{20}=-30.1\left[\mathrm{CHCl}_{3}, \mathrm{c}=1.00\right]$

HRMS (Cl) m/z: [M + H] $]^{+}$Calcd for $\mathrm{C}_{31} \mathrm{H}_{36} \mathrm{~N}_{3} \mathrm{O}_{5} \mathrm{~S}$ 562.2370; Found 562.2372

\section{Benzyl (S)-3-((S)-3-(4-chlorophenyl)-1-((2-(methylthio)phenyl)amino)-1-oxopropan-2-yl)-2,2,5-tri-} methyl-4-oxoimidazolidine-1-carboxylate (15d)

According to GP carbamate 13 (65.7 mg, $144 \mu \mathrm{mol}, 1.0$ eq.), 1-chloro-4-iodobenzene (68.8 mg, 288 $\mu \mathrm{mol}, 2.0$ eq.), silver carbonate $(80.0 \mathrm{mg}, 288 \mu \mathrm{mol}, 2.0$ eq.), dibenzyl phosphate $(8.0 \mathrm{mg}, 28.8 \mu \mathrm{mol}$, $20 \mathrm{~mol} \%)$ and $\mathrm{Pd}(\mathrm{OAc})_{2}(3.2 \mathrm{mg}, 14.3 \mu \mathrm{mol}, 10 \mathrm{~mol} \%)$ were reacted at $60^{\circ} \mathrm{C}$ for 2 days and $80^{\circ} \mathrm{C}$ for 1 day. The crude product was purified by column chromatography $\left(\mathrm{SiO}_{2}, \mathrm{PE}: \mathrm{EtOAc}\right.$ 100:0 $\rightarrow$ 80:20) and the product $15 \mathrm{~d}(57.7 \mathrm{mg}, 102 \mu \mathrm{mol}, 71 \%)$ was obtained as a white lyophilisate.

$\mathbf{R}_{\mathbf{f}}(\mathbf{1 5 d})=0.31\left(\mathrm{SiO}_{2}, \mathrm{PE}:\right.$ EtOAc $\left.70: 30\right)$

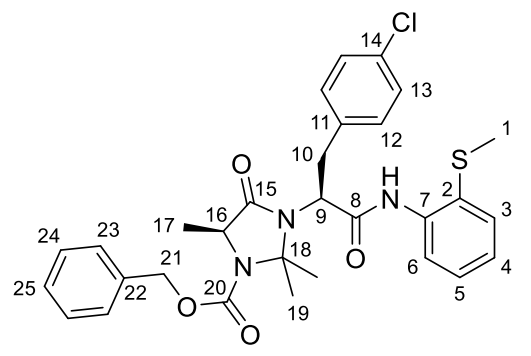

$15 d$

${ }^{1} \mathrm{H}-\mathrm{NMR}\left(500 \mathrm{MHz}\right.$, DMSO-d 6 , 373K): $\delta=1.07(\mathrm{~s}, 3 \mathrm{H}, 19-\mathrm{H}), 1.45\left(\mathrm{~d},{ }^{3} \mathrm{~J}_{17,16}=6.6 \mathrm{~Hz}, 3 \mathrm{H}, 17-\mathrm{H}\right), 1.61$ (s, $\left.3 \mathrm{H}, 19-\mathrm{H}^{\prime}\right), 2.42(\mathrm{~s}, 3 \mathrm{H}, 1-\mathrm{H}), 3.53\left(\mathrm{dd},{ }^{2} J_{10 \mathrm{a}, 10 \mathrm{~b}}=13.5 \mathrm{~Hz},{ }^{3} J_{10 \mathrm{a}, 9}=5.7 \mathrm{~Hz}, 1 \mathrm{H}, 10-\mathrm{H}_{\mathrm{a}}\right), 3.81\left(\mathrm{dd},{ }^{2} J_{10 \mathrm{~b}, 10 \mathrm{a}}=\right.$ $\left.13.5 \mathrm{~Hz},{ }^{3} J_{10 b, 9}=9.7 \mathrm{~Hz}, 1 \mathrm{H}, 10-\mathrm{H}_{\mathrm{b}}\right), 4.14\left(\mathrm{q},{ }^{3} \mathrm{~J}_{16,17}=6.6 \mathrm{~Hz}, 1 \mathrm{H}, 16-\mathrm{H}\right), 4.28\left(\mathrm{dd},{ }^{3} \mathrm{~g}_{9,10 \mathrm{~b}}=9.7 \mathrm{~Hz},{ }^{3} J_{9,10 \mathrm{a}}=\right.$ 
$5.7 \mathrm{~Hz}, 1 \mathrm{H}, 9-\mathrm{H}), 5.10\left(\mathrm{~d},{ }^{2} J_{21 \mathrm{a}, 21 \mathrm{~b}}=12.6 \mathrm{~Hz}, 1 \mathrm{H}, 21-\mathrm{H}_{\mathrm{a}}\right), 5.16\left(\mathrm{~d},{ }^{2} \mathrm{~J}_{21 \mathrm{~b}, 21 \mathrm{a}}=12.6 \mathrm{~Hz}, 1 \mathrm{H}, 21-\mathrm{H}_{\mathrm{b}}\right), 7.20$ (ddd, $\left.{ }^{3} J_{4,3}={ }^{3} J_{4,5}=7.5 \mathrm{~Hz},{ }^{4} J_{4,6}=1.6 \mathrm{~Hz}, 1 \mathrm{H}, 4-\mathrm{H}\right), 7.24\left(\mathrm{ddd},{ }^{3} J_{5,6}=8.8 \mathrm{~Hz},{ }^{3} J_{5,4}=7.5 \mathrm{~Hz},{ }^{4} J_{5,3}=1.6 \mathrm{~Hz}, 1 \mathrm{H}, 5-\mathrm{H}\right)$, $7.31-7.40(\mathrm{~m}, 9 \mathrm{H}, 12-\mathrm{H} / 13-\mathrm{H} / 23-\mathrm{H} / 24-\mathrm{H} / 25-\mathrm{H}), 7.45\left(\mathrm{dd},{ }^{3} J_{3,4}=7.5 \mathrm{~Hz},{ }^{4} J_{3,5}=1.6 \mathrm{~Hz}, 1 \mathrm{H}, 3-\mathrm{H}\right), 7.72$ (dd, $\left.{ }^{3} \sigma_{6,5}=8.8 \mathrm{~Hz},{ }^{4} J_{6,4}=1.6 \mathrm{~Hz}, 1 \mathrm{H}, 6-\mathrm{H}\right), 9.23(\mathrm{bs}, 1 \mathrm{H}, \mathrm{N}-\mathrm{H}$ ).

${ }^{13} \mathrm{C}-N M R$ (125 MHz, DMSO-d $\mathrm{d}_{6}$, 373K): $\delta=16.1$ (q, C-1), 17.9 (q, C-17), 23.1 (q, C-19), 25.3 (q, C-19'), 33.7 (t, C-10), 53.5 (d, C-16), 58.8 (d, C-9), 66.0 (t, C-21), 78.5 (s, C-18), 123.4 (d, C-6), 125.3 (d, C-4), 126.1 (d, C-5), 127.2 (d, C-23), 127.4 (d, C-25), 127.7 (d, C-13), 127.9 (d, C-24), 129.0 (d, C-3), 130.1 (s, C-2), 130.9 (d, C-12), 131.1 (s, C-14), 135.8 (s, C-7), 135.8 (s, C-22), 136.2 (s, C-11), 151.6 (s, C-20), 167.3 (s, C-8), $170.1(\mathrm{~s}, \mathrm{C}-15)$.

Optical rotation: $\quad[\alpha]_{D}^{20}=-24.4\left[\mathrm{CHCl}_{3}, \mathrm{c}=1.00\right]$

HRMS (Cl) m/z: $[\mathrm{M}+\mathrm{H}]^{+}$Calcd for $\mathrm{C}_{30} \mathrm{H}_{33} \mathrm{ClN}_{3} \mathrm{O}_{4} \mathrm{~S}$ 566.1875; Found 566.1859

Benzyl (S)-3-((S)-3-(4-bromophenyl)-1-((2-(methylthio)phenyl)amino)-1-oxopropan-2-yl)-2,2,5-trimethyl-4-oxoimidazolidine-1-carboxylate (15e)

According to GP carbamate 13 (70.7 mg, $155 \mu \mathrm{mol}, 1.0$ eq.), 1-bromo-4-iodobenzene $(88.0 \mathrm{mg}, 310$ $\mu \mathrm{mol}, 2.0$ eq.), silver carbonate $(86.0 \mathrm{mg}, 310 \mu \mathrm{mol}, 2.0$ eq.), dibenzyl phosphate $(8.6 \mathrm{mg}, 31.1 \mu \mathrm{mol}$, $20 \mathrm{~mol} \%)$ and $\mathrm{Pd}(\mathrm{OAc})_{2}(3.2 \mathrm{mg}, 15.6 \mu \mathrm{mol}, 10 \mathrm{~mol} \%)$ were reacted at $60^{\circ} \mathrm{C}$ for 2 days and $80^{\circ} \mathrm{C}$ for 2 days. The crude product was purified by column chromatography $\left(\mathrm{SiO}_{2}\right.$, Cyclohexane:EtOAc 100:0 $\rightarrow$ 60:40) and the product $15 \mathrm{e}(70.8 \mathrm{mg}, 116 \mu \mathrm{mol}, 75 \%)$ was obtained as a white lyophilisate.

$\mathbf{R}_{\mathbf{f}}(\mathbf{1 5 e})=0.15\left(\mathrm{SiO}_{2}, \mathrm{PE}:\right.$ EtOAc 80:20)

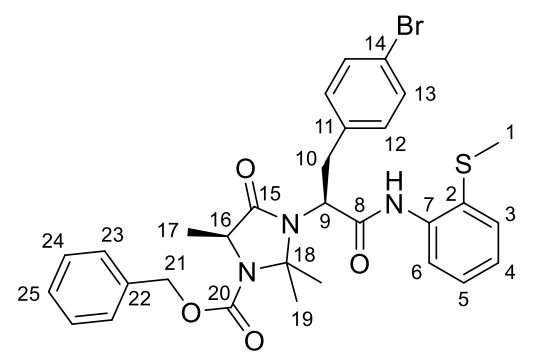

15 e

${ }^{1} \mathrm{H}-\mathrm{NMR}\left(500 \mathrm{MHz}\right.$, DMSO-d 6 , 373K): $\delta=1.08(\mathrm{~s}, 3 \mathrm{H}, 19-\mathrm{H}), 1.44$ (d, $\left.{ }^{3} \mathrm{~J}_{17,16}=6.6 \mathrm{~Hz}, 3 \mathrm{H}, 17-\mathrm{H}\right), 1.61$ (s, $\left.3 \mathrm{H}, 19-\mathrm{H}^{\prime}\right), 2.42(\mathrm{~s}, 3 \mathrm{H}, 1-\mathrm{H}), 3.52\left(\mathrm{dd},{ }^{2} \mathrm{~J}_{10 \mathrm{a}, 10 \mathrm{~b}}=13.5 \mathrm{~Hz},{ }^{3} \mathrm{~J}_{10 \mathrm{a}, 9}=5.7 \mathrm{~Hz}, 1 \mathrm{H}, 10-\mathrm{H}_{\mathrm{a}}\right), 3.79\left(\mathrm{dd},{ }^{2} \mathrm{~J}_{10 \mathrm{~b}, 10 \mathrm{a}}=\right.$ $\left.13.5 \mathrm{~Hz},{ }^{3} J_{10 b, 9}=9.7 \mathrm{~Hz}, 2 \mathrm{H}, 10-\mathrm{H}_{\mathrm{b}}\right), 4.14\left(\mathrm{q},{ }^{3} \mathrm{~J}_{16,17}=6.6 \mathrm{~Hz}, 1 \mathrm{H}, 16-\mathrm{H}\right), 4.27$ (dd, ${ }^{3} \mathrm{~J}_{9,10 \mathrm{~b}}=9.7 \mathrm{~Hz},{ }^{3} \int_{9,10 \mathrm{a}}=$ $5.7 \mathrm{~Hz}, 1 \mathrm{H}, 9-\mathrm{H}), 5.10\left(\mathrm{~d},{ }^{2} J_{21 \mathrm{a}, 21 \mathrm{~b}}=12.6 \mathrm{~Hz}, 1 \mathrm{H}, 21-\mathrm{H}_{\mathrm{a}}\right), 5.16\left(\mathrm{~d},{ }^{2} \mathrm{~J}_{21 \mathrm{~b}, 21 \mathrm{a}}=12.6 \mathrm{~Hz}, 1 \mathrm{H}, 21-\mathrm{H}_{\mathrm{b}}\right), 7.20$ (ddd, $\left.{ }^{3} J_{4,3}={ }^{3} J_{4,5}=7.5 \mathrm{~Hz},{ }^{4} J_{4,6}=1.6 \mathrm{~Hz}, 1 \mathrm{H}, 4-\mathrm{H}\right), 7.24\left(\mathrm{ddd},{ }^{3} J_{5,6}=7.9 \mathrm{~Hz},{ }^{3} J_{5,4}=7.5 \mathrm{~Hz},{ }^{4} J_{5,3}=1.6 \mathrm{~Hz}, 1 \mathrm{H}, 5-\mathrm{H}\right.$ ), $7.29-7.40(\mathrm{~m}, 7 \mathrm{H}, 12-\mathrm{H} / 23-\mathrm{H} / 24-\mathrm{H} / 25-\mathrm{H}), 7.45\left(\mathrm{dd},{ }^{3} \mathrm{~J}_{3,4}=7.5 \mathrm{~Hz},{ }^{4} J_{3,5}=1.6 \mathrm{~Hz}, 1 \mathrm{H}, 3-\mathrm{H}\right), 7.49(\mathrm{~m}, 2 \mathrm{H}$, 13-H), $7.72\left(\mathrm{dd},{ }^{3} J_{6,5}=7.9 \mathrm{~Hz},{ }^{4} J_{6,4}=1.6 \mathrm{~Hz}, 1 \mathrm{H}, 6-\mathrm{H}\right.$ ), 9.22 (bs, $1 \mathrm{H}, \mathrm{N}-\mathrm{H}$ ).

${ }^{13}$ C-NMR (125 MHz, DMSO-d 6 , 373K): $\delta=16.1$ (q, C-1), 17.9 (q, C-17), 23.1 (q, C-19), 25.3 (q, C-19'), 33.7 (t, C-10), 53.5 (d, C-16), 58.8 (d, C-9), 66.0 (t, C-21), 78.5 (s, C-18), 119.3 (s, C-14), 123.4 (d, C-6), 125.3 (d, C-4), 126.1 (d, C-5), 127.2 (d, C-23), 127.4 (d, C-25), 127.9 (d, C-24), 129.0 (d, C-3), 130.1 (s, 
C-2), 130.6 (d, C-13), 131.3 (d, C-12), 135.8 (s, C-7), 135.8 (s, C-22), 136.6 (s, C-11), 151.6 (s, C-20), 167.2 (s, C-8), 170.1 (s, C-15).

Optical rotation: $\quad[\alpha]_{D}^{20}=-25.3\left[\mathrm{CHCl}_{3}, \mathrm{c}=1.00\right]$

HRMS (Cl) m/z: [M + H] $]^{+}$Calcd for $\mathrm{C}_{30} \mathrm{H}_{33} \mathrm{BrN}_{3} \mathrm{O}_{4} \mathrm{~S}$ 610.1370; Found 610.1352

Benzyl (S)-3-((S)-3-(4-iodophenyl)-1-((2-(methylthio)phenyl)amino)-1-oxopropan-2-yl)-2,2,5-trimethyl-4-oxoimidazolidine-1-carboxylate (15f)

According to GP carbamate 13 (69.3 mg, $152 \mu \mathrm{mol}, 1.0$ eq.), 1,4-diiodobenzene (100 mg, $304 \mu \mathrm{mol}, 2.0$ eq.), silver carbonate ( $84.0 \mathrm{mg}, 304 \mu \mathrm{mol}, 2.0$ eq.), dibenzyl phosphate $(8.5 \mathrm{mg}, 30.4 \mu \mathrm{mol}, 20 \mathrm{~mol} \%)$ and $\mathrm{Pd}(\mathrm{OAc})_{2}(3.4 \mathrm{mg}, 15.2 \mu \mathrm{mol}, 10 \mathrm{~mol} \%)$ were reacted at $60^{\circ} \mathrm{C}$ for 2 days. The crude product was purified by column chromatography $\left(\mathrm{SiO}_{2}, \mathrm{PE}:\right.$ EtOAc $\left.100: 0 \rightarrow 60: 40\right)$ and the product $\mathbf{1 5 f}(51.9 \mathrm{mg}$, $78.9 \mu \mathrm{mol}, 52 \%)$ was obtained as a yellowish resin.

$\mathbf{R}_{\mathbf{f}}(\mathbf{1 5 h})=0.37\left(\mathrm{SiO}_{2}, \mathrm{PE}:\right.$ EtOAc 70:30)

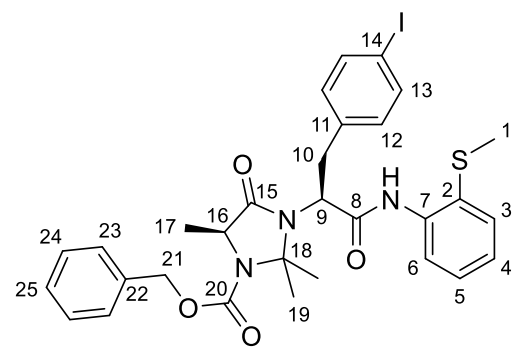

$15 h$

\section{Major rotamer:}

${ }^{1} \mathrm{H}-\mathrm{NMR}\left(400 \mathrm{MHz}\right.$, DMSO-d 6 ): $\delta=0.93(\mathrm{~s}, 3 \mathrm{H}, 19-\mathrm{H}), 1.39\left(\mathrm{~d},{ }^{3} \mathrm{~J}_{17,16}=6.1 \mathrm{~Hz}, 3 \mathrm{H}, 17-\mathrm{H}\right), 1.53(\mathrm{~s}, 3 \mathrm{H}$, $\left.19-\mathrm{H}^{\prime}\right), 2.43(\mathrm{~s}, 3 \mathrm{H}, 1-\mathrm{H}), 3.42\left(\mathrm{dd},{ }^{2} J_{10 \mathrm{a}, 10 \mathrm{~b}}=13.1 \mathrm{~Hz},{ }^{3} J_{10 \mathrm{a}, 9}=4.9 \mathrm{~Hz}, 1 \mathrm{H}, 10-\mathrm{H}_{\mathrm{a}}\right), 3.83\left(\mathrm{dd},{ }^{2} J_{10 \mathrm{~b}, 10 \mathrm{a}}=13.1\right.$ $\left.\mathrm{Hz},{ }^{3} J_{10 \mathrm{~b}, 9}=11.0 \mathrm{~Hz}, 2 \mathrm{H}, 10-\mathrm{H}_{\mathrm{b}}\right), 4.09\left(\mathrm{q},{ }^{3} J_{16,17}=6.1 \mathrm{~Hz}, 1 \mathrm{H}, 16-\mathrm{H}\right), 4.28(\mathrm{~m}, 1 \mathrm{H}, 9-\mathrm{H}), 5.03\left(\mathrm{~d},{ }^{2} J_{21 \mathrm{a}, 21 \mathrm{~b}}=\right.$ $\left.12.5 \mathrm{~Hz}, 1 \mathrm{H}, 21-\mathrm{H}_{\mathrm{a}}\right), 5.13\left(\mathrm{~d},{ }^{2} \mathrm{~J}_{21 \mathrm{~b}, 21 \mathrm{a}}=12.5 \mathrm{~Hz}, 1 \mathrm{H}, 21-\mathrm{H}_{\mathrm{b}}\right), 7.15\left(\mathrm{~d},{ }^{3} J_{12,13}=8.0 \mathrm{~Hz}, 2 \mathrm{H}, 12-\mathrm{H}\right), 7.19-$ $7.26(\mathrm{~m}, 2 \mathrm{H}, 4-\mathrm{H} / 5-\mathrm{H}), 7.30-7.42(\mathrm{~m}, 6 \mathrm{H}, 3-\mathrm{H} / 23-\mathrm{H} / 24-\mathrm{H} / 25-\mathrm{H}), 7.56(\mathrm{~m}, 1 \mathrm{H}, 6-\mathrm{H}), 7.67\left(\mathrm{~d},{ }^{3} \mathrm{~J}_{13,12}=\right.$ $8.0 \mathrm{~Hz}, 2 \mathrm{H}, 13-\mathrm{H}), 9.42$ (bs, $1 \mathrm{H}, \mathrm{N}-\mathrm{H})$.

${ }^{13} \mathrm{C}-N M R\left(100 \mathrm{MHz}\right.$, DMSO-d $\mathrm{d}_{6}$ ): $\delta=15.6$ (q, C-1), 18.9 (q, C-17), 23.0 (q, C-19), 25.0 (q, C-19'), 34.0 (t, C-10), 53.8 (d, C-16), 58.5 (d, C-9), 66.2 (t, C-21), 78.6 (s, C-18), 92.5 (s, C-14), 125.0 (d, C-6), 125.8 (d, C-4), 126.3 (d, C-5), 127.6 (d, C-3), 127.8 (d, C-23), 128.0 (d, C-25), 128.5 (d, C-24), 132.0 (d, C-12), 132.1 (s, C-2), 135.1 (s, C-7), 136.2 (s, C-22), 137.0 (d, C-13), 137.6 (s, C-11), 151.7 (s, C-20), 167.6 (s, C-8), $170.3(\mathrm{~s}, \mathrm{C}-15)$.

Minor rotamer (selected signals):

${ }^{1} \mathrm{H}-\mathrm{NMR}\left(400 \mathrm{MHz}, \mathrm{DMSO}-\mathrm{d}_{6}\right): \delta=0.84(\mathrm{~s}, 3 \mathrm{H}, 19-\mathrm{H})$.

${ }^{13} \mathrm{C}-\mathrm{NMR}\left(100 \mathrm{MHz}, \mathrm{DMSO}-\mathrm{d}_{6}\right): \delta=17.5$ (q, C-17).

Optical rotation:

$$
[\alpha]_{D}^{20}=-25.1\left[\mathrm{CHCl}_{3}, \mathrm{c}=1.00\right]
$$


HRMS (Cl) m/z: [M] $]^{+}$Calcd for $\mathrm{C}_{30} \mathrm{H}_{32} \mathrm{IN}_{3} \mathrm{O}_{4} \mathrm{~S}$ 657.1153; Found 657.1157

Benzyl (S)-3-((S)-3-(2-fluorophenyl)-1-((2-(methylthio)phenyl)amino)-1-oxopropan-2-yl)-2,2,5-trimethyl-4-oxoimidazolidine-1-carboxylate $(15 \mathrm{~g})$

According to GP carbamate 13 (71.1 mg, $156 \mu \mathrm{mol}, 1.0$ eq.), 1-fluoro-2-iodobenzene ( $36.4 \mu \mathrm{L}, 69.3 \mathrm{mg}$, $312 \mu \mathrm{mol}, 2.0$ eq.), silver carbonate $(86.0 \mathrm{mg}, 312 \mu \mathrm{mol}, 2.0$ eq.), dibenzyl phosphate $(8.7 \mathrm{mg}, 31.2$ $\mu \mathrm{mol}, 20 \mathrm{~mol} \%)$ and $\mathrm{Pd}(\mathrm{OAc})_{2}(3.5 \mathrm{mg}, 15.6 \mu \mathrm{mol}, 10 \mathrm{~mol} \%)$ were reacted at $60^{\circ} \mathrm{C}$ for 1 day and $80{ }^{\circ} \mathrm{C}$ for 2 days. The crude product was purified by column chromatography $\left(\mathrm{SiO}_{2}, \mathrm{Cyclohexane:EtOAc} \mathrm{100:0}\right.$ $\rightarrow 60: 40)$ and the product $15 \mathrm{~g}(54.4 \mathrm{mg}, 99.0 \mu \mathrm{mol}, 63 \%)$ was obtained as a white lyophilisate.

$\mathbf{R}_{\mathbf{f}}(\mathbf{1 5 g})=0.38\left(\mathrm{SiO}_{2}, \mathrm{PE}:\right.$ EtOAc 70:30)

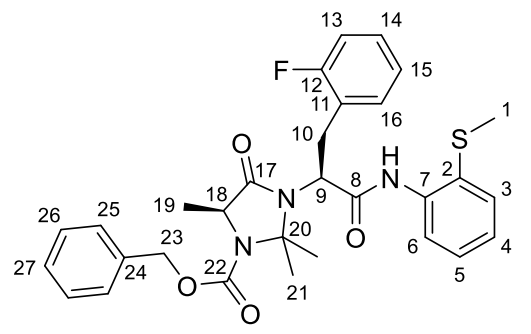

$15 g$

${ }^{1} \mathrm{H}-\mathrm{NMR}\left(500 \mathrm{MHz}\right.$, DMSO-d 6 , 373K): $\delta=1.16(\mathrm{~s}, 3 \mathrm{H}, 21-\mathrm{H}), 1.46$ (d, $\left.{ }^{3} \mathrm{~J}_{19,18}=6.6 \mathrm{~Hz}, 3 \mathrm{H}, 19-\mathrm{H}\right), 1.65$ (s, $\left.3 \mathrm{H}, 21-\mathrm{H}^{\prime}\right), 2.42(\mathrm{~s}, 3 \mathrm{H}, 1-\mathrm{H}), 3.70\left(\mathrm{dd},{ }^{2} \mathrm{~J}_{10 \mathrm{a}, 10 \mathrm{~b}}=13.8 \mathrm{~Hz},{ }^{3} J_{10 \mathrm{a}, 9}=6.9 \mathrm{~Hz}, 1 \mathrm{H}, 10-\mathrm{H}_{\mathrm{a}}\right), 3.74\left(\mathrm{dd},{ }^{2} J_{10 \mathrm{~b}, 10 \mathrm{a}}=\right.$ $\left.13.8 \mathrm{~Hz},{ }^{3} \mathrm{~J}_{10 \mathrm{~b}, 9}=8.8 \mathrm{~Hz}, 2 \mathrm{H}, 10-\mathrm{H}_{\mathrm{b}}\right), 4.16\left(\mathrm{q},{ }^{3} \mathrm{~J}_{18,19}=6.6 \mathrm{~Hz}, 1 \mathrm{H}, 18-\mathrm{H}\right), 4.24\left(\mathrm{dd},{ }^{3} \mathrm{~J}_{9,10 \mathrm{~b}}=8.8 \mathrm{~Hz},{ }^{3} J_{9,10 \mathrm{a}}=\right.$ $6.9 \mathrm{~Hz}, 1 \mathrm{H}, 9-\mathrm{H}), 5.12\left(\mathrm{~d},{ }^{2} J_{23 \mathrm{a}, 23 \mathrm{~b}}=12.6 \mathrm{~Hz}, 1 \mathrm{H}, 23-\mathrm{H}_{\mathrm{a}}\right), 5.17\left(\mathrm{~d},{ }^{2} J_{23 \mathrm{~b}, 23 \mathrm{a}}=12.6 \mathrm{~Hz}, 1 \mathrm{H}, 23-\mathrm{H}_{\mathrm{b}}\right), 7.12-$ $7.17\left(\mathrm{~m}, 2 \mathrm{H}, 13-\mathrm{H} / 15-\mathrm{H}\right.$ ), 7.19 (ddd, ${ }^{3} \mathrm{~J}_{4,3}=7.9 \mathrm{~Hz},{ }^{3} J_{4,5}=7.5 \mathrm{~Hz},{ }^{4} \mathrm{~J}_{4,6}=1.6 \mathrm{~Hz}, 1 \mathrm{H}, 4-\mathrm{H}$ ), 7.25 (ddd, ${ }^{3} J_{5,6}=$ $\left.8.2 \mathrm{~Hz},{ }^{3} J_{5,4}=7.5 \mathrm{~Hz},{ }^{4} J_{5,3}=1.6 \mathrm{~Hz}, 1 \mathrm{H}, 5-\mathrm{H}\right), 7.27-7.40(\mathrm{~m}, 7 \mathrm{H}, 14-\mathrm{H} / 16-\mathrm{H} / 25-\mathrm{H} / 26-\mathrm{H} / 27-\mathrm{H}), 7.46$ (dd, $\left.{ }^{3} J_{3,4}=7.9 \mathrm{~Hz},{ }^{4} J_{3,5}=1.6 \mathrm{~Hz}, 1 \mathrm{H}, 3-\mathrm{H}\right), 7.77\left(\mathrm{dd},{ }^{3} J_{6,5}=8.2 \mathrm{~Hz},{ }^{4} J_{6,4}=1.6 \mathrm{~Hz}, 1 \mathrm{H}, 6-\mathrm{H}\right), 9.23(\mathrm{bs}, 1 \mathrm{H}, \mathrm{N}-\mathrm{H})$.

${ }^{13}$ C-NMR (125 MHz, DMSO-d 6 , 373K): $\delta=16.4$ (q, C-1), 17.9 (q, C-19), 22.6 (q, C-21), 25.6 (q, C-21'), $28.0\left(\mathrm{dt},{ }^{3} J_{10, F}=2.2 \mathrm{~Hz}, \mathrm{C}-10\right), 53.4(\mathrm{~d}, \mathrm{C}-18), 57.9$ (d, C-9), 66.0 (t, C-23), 78.6 (s, C-20), 114.6 (dd, ${ }^{2} J_{13, F}=$ $22.6 \mathrm{~Hz}, \mathrm{C}-13$ ), 122.9 (d, C-6), 123.7 (d, ${ }^{2} J_{11, F}=14.6 \mathrm{~Hz}, \mathrm{C}-11$ ), 123.8 (dd, $\left.{ }^{4} J_{15, F}=3.2 \mathrm{~Hz}, \mathrm{C}-15\right), 125.1$ (d, C-4), 126.2 (d, C-5), 127.2 (d, C-25), 127.4 (d, C-27), 127.9 (d, C-26), 128.5 (dd, ${ }^{3} J_{16, F}=7.5$ Hz, C-16), 129.4 (d, C-3), 129.6 (s, C-2), 131.5 (dd, ${ }^{3} \mathrm{~J}_{14, \mathrm{~F}}=4.3 \mathrm{~Hz}, \mathrm{C}-14$ ), 135.8 (s, C-24), 136.0 (s, C-7), 151.6 (s, C22), $160.6\left(\mathrm{~d},{ }^{1} J_{12, \mathrm{~F}}=243.9 \mathrm{~Hz}, \mathrm{C}-12\right), 167.1$ (s, C-8), 170.1 (s, C-17).

Optical rotation: $\quad[\alpha]_{D}^{20}=+1.4\left[\mathrm{CHCl}_{3}, \mathrm{c}=1.00\right]$

HRMS (Cl) m/z: [M + H] ${ }^{+}$Calcd for $\mathrm{C}_{30} \mathrm{H}_{33} \mathrm{FN}_{3} \mathrm{O}_{4} \mathrm{~S}$ 550.2170; Found 550.2147

Benzyl (S)-3-((S)-3-(4-formylphenyl)-1-((2-(methylthio)phenyl)amino)-1-oxopropan-2-yl)-2,2,5-trimethyl-4-oxoimidazolidine-1-carboxylate (15h)

According to GP carbamate 13 (70.8 mg, $155 \mu \mathrm{mol}, 1.0$ eq.), 4-iodobenzaldehyde (72.1 mg, $311 \mu \mathrm{mol}$, 2.0 eq.), silver carbonate $(86.0 \mathrm{mg}, 311 \mu \mathrm{mol}, 2.0$ eq.), dibenzyl phosphate $(8.7 \mathrm{mg}, 31.1 \mu \mathrm{mol}, 20$ 
mol\%) and $\mathrm{Pd}(\mathrm{OAc})_{2}(3.5 \mathrm{mg}, 15.5 \mu \mathrm{mol}, 10 \mathrm{~mol} \%)$ were reacted at $60{ }^{\circ} \mathrm{C}$ for 2 days. The crude product was purified by column chromatography $\left(\mathrm{SiO}_{2}\right.$, Cyclohexane:EtOAc 100:0 $\rightarrow$ 40:60) and the product $15 \mathrm{~h}(47.8 \mathrm{mg}, 85.4 \mu \mathrm{mol}, 55 \%)$ was obtained as a white lyophilisate.

$\mathbf{R}_{\mathbf{f}}(\mathbf{1 5 i})=0.15\left(\mathrm{SiO}_{2}, \mathrm{PE}:\right.$ EtOAc 70:30)

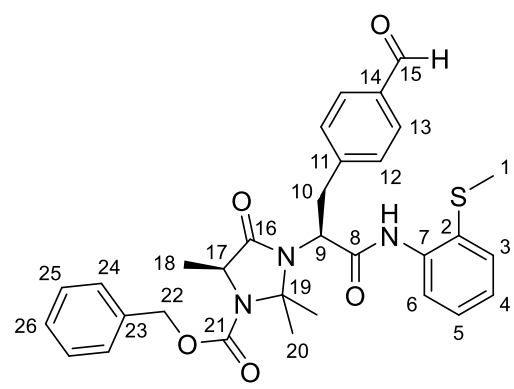

$15 \mathbf{i}$

\section{Major rotamer:}

${ }^{1} \mathrm{H}-\mathrm{NMR}\left(400 \mathrm{MHz}\right.$, DMSO-d 6 ): $\delta=0.90(\mathrm{~s}, 3 \mathrm{H}, 20-\mathrm{H}), 1.39\left(\mathrm{~d},{ }^{3} \mathrm{~J}_{18,17}=6.2 \mathrm{~Hz}, 3 \mathrm{H}, 18-\mathrm{H}\right), 1.54(\mathrm{~s}, 3 \mathrm{H}$, $\left.20-\mathrm{H}^{\prime}\right), 2.43(\mathrm{~s}, 3 \mathrm{H}, 1-\mathrm{H}), 3.57\left(\mathrm{dd},{ }^{2} J_{10 a, 10 \mathrm{~b}}=13.0 \mathrm{~Hz},{ }^{3} J_{10 \mathrm{a}, 9}=4.9 \mathrm{~Hz}, 1 \mathrm{H}, 10-\mathrm{H}_{\mathrm{a}}\right), 3.77 \quad\left(\mathrm{dd},{ }^{2} J_{10 \mathrm{~b}, 10 \mathrm{a}}=\right.$ $\left.13.0 \mathrm{~Hz},{ }^{3} J_{10 \mathrm{~b}, 9}=11.0 \mathrm{~Hz}, 2 \mathrm{H}, 10-\mathrm{H}_{\mathrm{b}}\right), 4.10\left(\mathrm{q},{ }^{3} \mathrm{~J}_{17,18}=6.2 \mathrm{~Hz}, 1 \mathrm{H}, 17-\mathrm{H}\right), 4.38(\mathrm{~m}, 1 \mathrm{H}, 9-\mathrm{H}), 5.01(\mathrm{~d}$, $\left.{ }^{2} J_{21 \mathrm{a}, 21 \mathrm{~b}}=12.6 \mathrm{~Hz}, 1 \mathrm{H}, 22-\mathrm{H}_{\mathrm{a}}\right), 5.12\left(\mathrm{~d},{ }^{2} \mathrm{~J}_{21 \mathrm{~b}, 21 \mathrm{a}}=12.6 \mathrm{~Hz}, 1 \mathrm{H}, 22-\mathrm{H}_{\mathrm{b}}\right), 7.19-7.27(\mathrm{~m}, 2 \mathrm{H}, 4-\mathrm{H} / 5-\mathrm{H}), 7.29$ $-7.42(\mathrm{~m}, 6 \mathrm{H}, 3-\mathrm{H} / 24-\mathrm{H} / 25-\mathrm{H} / 26-\mathrm{H}), 7.54-7.60(\mathrm{~m}, 3 \mathrm{H}, 6-\mathrm{H} / 12-\mathrm{H}), 7.87\left(\mathrm{~d},{ }^{3} \mathrm{~J}_{13,12}=8.0 \mathrm{~Hz}, 2 \mathrm{H}, 13-\mathrm{H}\right)$, 9.46 (bs, $1 \mathrm{H}, \mathrm{N}-\mathrm{H}), 9.98(\mathrm{~s}, 1 \mathrm{H}, 15-\mathrm{H})$.

${ }^{13}$ C-NMR (100 MHz, DMSO-d (1) ) $\delta=15.6$ (q, C-1), 18.9 (q, C-18), 23.0 (q, C-20), 25.0 (q, C-20'), 34.6 (t, C-10), 53.8 (d, C-17), 58.2 (d, C-9), 66.2 (t, C-22), 78.6 (s, C-19), 125.0 (d, C-6), 125.8 (d, C-4), 126.3 (d, C-5), 127.6 (d, C-3), 127.8 (d, C-24), 128.0 (d, C-26), 128.5 (d, C-25), 129.6 (d, C-13), 130.4 (d, C-12), 132.2 (s, C-2), 134.8 (s, C-14), 135.1 (s, C-7), 136.2 (s, C-23), 145.1 (s, C-11), 151.7 (s, C-21), 167.5 (s, C8), $170.4(\mathrm{~s}, \mathrm{C}-16), 192.8$ (s, C-15).

Minor rotamer (selected signals):

${ }^{1} \mathrm{H}-\mathrm{NMR}(400 \mathrm{MHz}$, DMSO-d 6 ): $\delta=0.81(\mathrm{~s}, 3 \mathrm{H}, 20-\mathrm{H})$.

Optical rotation: $\quad[\alpha]_{D}^{20}=-35.3\left[\mathrm{CHCl}_{3}, \mathrm{c}=1.00\right]$

HRMS (Cl) m/z: [M + H] $]^{+}$Calcd for $\mathrm{C}_{31} \mathrm{H}_{34} \mathrm{~N}_{3} \mathrm{O}_{5} \mathrm{~S}$ 560.2214; Found 560.2223

Benzyl (S)-2,2,5-trimethyl-3-((S)-1-((2-(methylthio)phenyl)amino)-3-(4-nitrophenyl)-1-oxopropan-2yl)-4-oxoimidazolidine-1-carboxylate (15i)

According to GP carbamate 13 ( $68.6 \mathrm{mg}, 151 \mu \mathrm{mol}, 1.0$ eq.), 1-iodo-4-nitrobenzene ( $75.0 \mathrm{mg}, 301 \mu \mathrm{mol}$, 2.0 eq.), silver carbonate ( $83.0 \mathrm{mg}, 301 \mu \mathrm{mol}, 2.0$ eq.), dibenzyl phosphate $(8.4 \mathrm{mg}, 30.1 \mu \mathrm{mol}, 20$ mol\%) and $\mathrm{Pd}(\mathrm{OAc})_{2}(3.4 \mathrm{mg}, 15.1 \mu \mathrm{mol}, 10 \mathrm{~mol} \%)$ were reacted at $60^{\circ} \mathrm{C}$ for 2 days and at $80{ }^{\circ} \mathrm{C}$ for 1 day. The crude product was purified by normal phase column chromatography $\left(\mathrm{SiO}_{2}, \mathrm{PE}\right.$ :EtOAc 100:0 $\rightarrow$ 60:40) followed by reverse phase column chromatography ( $\mathrm{C} 18, \mathrm{H}_{2} \mathrm{O}: \mathrm{MeCN}$ 100:0 $\rightarrow$ 0:100) and the product $15 \mathrm{i}(48.9 \mathrm{mg}, 84.8 \mu \mathrm{mol}, 56 \%)$ was obtained as a yellowish lyophilisate. 
$\mathbf{R}_{\mathbf{f}}(\mathbf{1 5 j})=0.24\left(\mathrm{SiO}_{2}, \mathrm{PE}:\right.$ EtOAC 70:30)

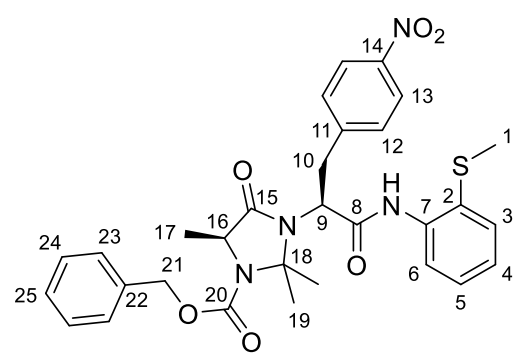

15j

\section{Major rotamer:}

${ }^{1} \mathrm{H}-\mathrm{NMR}\left(500 \mathrm{MHz}\right.$, DMSO-d 6 ): $\delta=0.93(\mathrm{~s}, 3 \mathrm{H}, 19-\mathrm{H}), 1.38\left(\mathrm{~d},{ }^{3} \mathrm{~J}_{17,16}=6.6 \mathrm{~Hz}, 3 \mathrm{H}, 17-\mathrm{H}\right), 1.53(\mathrm{~s}, 3 \mathrm{H}$, $\left.19-\mathrm{H}^{\prime}\right), 2.44(\mathrm{~s}, 3 \mathrm{H}, 1-\mathrm{H}), 3.60\left(\mathrm{dd},{ }^{2} J_{10 \mathrm{a}, 10 \mathrm{~b}}=12.9 \mathrm{~Hz},{ }^{3} \mathrm{~J}_{10 \mathrm{a}, 9}=4.7 \mathrm{~Hz}, 1 \mathrm{H}, 10-\mathrm{H}_{\mathrm{a}}\right), 4.03\left(\mathrm{dd},{ }^{2} \mathrm{~J}_{10 \mathrm{~b}, 10 \mathrm{a}}=12.9\right.$ $\left.\mathrm{Hz},{ }^{3} J_{10 \mathrm{~b}, 9}=11.0 \mathrm{~Hz}, 2 \mathrm{H}, 10-\mathrm{H}_{\mathrm{b}}\right), 4.10\left(\mathrm{q},{ }^{3} \mathrm{~J}_{16,17}=6.6 \mathrm{~Hz}, 1 \mathrm{H}, 16-\mathrm{H}\right), 4.41(\mathrm{~m}, 1 \mathrm{H}, 9-\mathrm{H}), 5.00\left(\mathrm{~d},{ }^{2} \mathrm{~J}_{21 \mathrm{a}, 21 \mathrm{~b}}=\right.$ $\left.12.6 \mathrm{~Hz}, 1 \mathrm{H}, 21-\mathrm{H}_{\mathrm{a}}\right), 5.12\left(\mathrm{~d},{ }^{2} \mathrm{~J}_{21 \mathrm{~b}, 21 \mathrm{a}}=12.6 \mathrm{~Hz}, 1 \mathrm{H}, 21-\mathrm{H}_{\mathrm{b}}\right), 7.22\left(\mathrm{ddd},{ }^{3} J_{4,3}={ }^{3} J_{4,5}=7.6 \mathrm{~Hz},{ }^{4} J_{4,6}=1.6 \mathrm{~Hz}\right.$, $1 \mathrm{H}, 4-\mathrm{H}$ ), 7.25 (ddd, ${ }^{3} J_{5,4}=7.6 \mathrm{~Hz},{ }^{3} J_{5,6}=7.3 \mathrm{~Hz},{ }^{4} J_{5,3}=1.9 \mathrm{~Hz}, 1 \mathrm{H}, 5-\mathrm{H}$ ), $7.29-7.39$ (sh, $5 \mathrm{H}, 23-\mathrm{H} / 24-$ $\mathrm{H} / 25-\mathrm{H}$ ), 7.39 (dd, $\left.{ }^{3} J_{3,4}=7.6 \mathrm{~Hz},{ }^{4} J_{3,5}=1.9 \mathrm{~Hz}, 1 \mathrm{H}, 3-\mathrm{H}\right), 7.53\left(\mathrm{dd},{ }^{3} J_{6,5}=7.3 \mathrm{~Hz},{ }^{4} J_{6,4}=1.6 \mathrm{~Hz}, 1 \mathrm{H}, 6-\mathrm{H}\right.$ ), $7.62\left(\mathrm{~d},{ }^{3} \mathrm{~J}_{12,13}=8.4 \mathrm{~Hz}, 2 \mathrm{H}, 12-\mathrm{H}\right), 8.20\left(\mathrm{~d},{ }^{3} \mathrm{~J}_{13,12}=8.4 \mathrm{~Hz}, 2 \mathrm{H}, 13-\mathrm{H}\right), 9.48(\mathrm{bs}, 1 \mathrm{H}, \mathrm{N}-\mathrm{H})$.

${ }^{13}$ C-NMR (125 MHz, DMSO-d f $_{6}$ ) $\delta=15.5$ (q, C-1), 18.9 (q, C-17), 23.2 (q, C-19), 24.9 (q, C-19'), 34.4 (t, C-10), 53.8 (s, C-16), 57.9 (s, C-9), 66.3 (t, C-21), 78.6 (s, C-18), 123.5 (d, C-13), 125.2 (d, C-6), 125.8 (d, C-4), 126.4 (d, C-5), 127.5 (d, C-3), 127.8 (d, C-23), 128.0 (d, C-25), 128.5 (d, C-24), 130.9 (d, C-12), 132.4 (s, C-2), 134.9 (s, C-7), 136.3 (s, C-22), 146.2 (s, C-11), 146.4 (s, C-14), 151.7 (s, C-20), 167.4 (s, C-8), $170.5(\mathrm{~s}, \mathrm{C}-15)$.

Minor rotamer (selected signals):

${ }^{1} \mathrm{H}-\mathrm{NMR}$ (500 MHz, DMSO-d $\left.\mathrm{d}_{6}\right): \delta=0.83(\mathrm{~s}, 3 \mathrm{H}, 19-\mathrm{H}), 1.49\left(\mathrm{~s}, 3 \mathrm{H}, 19-\mathrm{H}^{\prime}\right)$.

Optical rotation: $\quad[\alpha]_{D}^{20}=-30.0\left[\mathrm{CHCl}_{3}, \mathrm{c}=1.00\right]$

HRMS (Cl) m/z: [M + H] $]^{+}$Calcd for $\mathrm{C}_{30} \mathrm{H}_{33} \mathrm{~N}_{4} \mathrm{O}_{6} \mathrm{~S}$ 577.2115; Found 577.2122

\section{Benzyl (S)-3-((S)-3-(1H-indol-5-yl)-1-((2-(methylthio)phenyl)amino)-1-oxopropan-2-yl)-2,2,5-trime-} thyl-4-oxoimidazolidine-1-carboxylate (15k)

According to GP carbamate 13 (66.3 mg, $146 \mu \mathrm{mol}, 1.0$ eq.), 5-iodo-1H-indole (70.7 mg, $291 \mu \mathrm{mol}, 2.0$ eq.), silver carbonate ( $80.0 \mathrm{mg}, 291 \mu \mathrm{mol}, 2.0$ eq.), dibenzyl phosphate ( $8.1 \mathrm{mg}, 29.1 \mu \mathrm{mol}, 20 \mathrm{~mol} \%)$ and $\mathrm{Pd}(\mathrm{OAc})_{2}(3.3 \mathrm{mg}, 14.6 \mu \mathrm{mol}, 10 \mathrm{~mol} \%)$ were reacted at $60^{\circ} \mathrm{C}$ for 2 days. The crude product was purified successively by column chromatography $\left(\mathrm{SiO}_{2}, \mathrm{PE}:\right.$ EtOAc 100:0 $\rightarrow$ 80:20) and preparative HPLC (Phenomenex Luna $5 \mu \mathrm{m}, \mathrm{H}_{2} \mathrm{O}: \mathrm{MeCN}$ 100:0 $\rightarrow$ 0:100) and the product 15k (47.7 mg, 83.6 $\mu \mathrm{mol}, 57 \%$ ) was obtained as a yellowish resin.

$\mathbf{R}_{\mathbf{f}}(\mathbf{1 5 k})=0.13\left(\mathrm{SiO}_{2}, \mathrm{PE}:\right.$ EtOAc 70:30) 


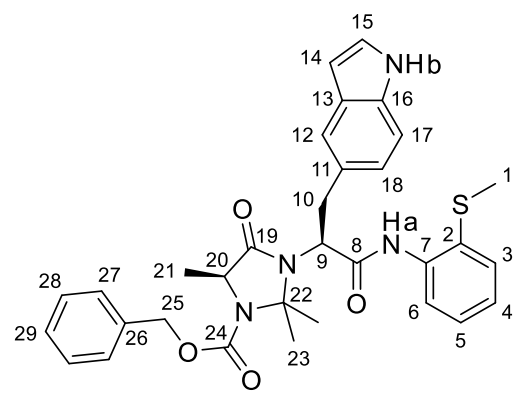

$15 \mathrm{k}$

${ }^{1} \mathrm{H}-\mathrm{NMR}\left(500 \mathrm{MHz}\right.$, DMSO-d 6 , 373K): $\delta=0.97(\mathrm{~s}, 3 \mathrm{H}, 23-\mathrm{H}), 1.46\left(\mathrm{~d},{ }^{3} \mathrm{~J}_{21,20}=6.6 \mathrm{~Hz}, 3 \mathrm{H}, 21-\mathrm{H}\right), 1.60$ (s, $\left.3 \mathrm{H}, 23-\mathrm{H}^{\prime}\right), 2.42(\mathrm{~s}, 3 \mathrm{H}, 1-\mathrm{H}), 3.61\left(\mathrm{dd},{ }^{2} J_{10 \mathrm{a}, 10 \mathrm{~b}}=13.5 \mathrm{~Hz},{ }^{3} J_{10 \mathrm{a}, 9}=6.0 \mathrm{~Hz}, 1 \mathrm{H}, 10-\mathrm{H}_{\mathrm{a}}\right), 3.81\left(\mathrm{dd},{ }^{2} J_{10 \mathrm{~b}, 10 \mathrm{a}}=\right.$ $\left.13.5 \mathrm{~Hz},{ }^{3} J_{10 b, 9}=9.4 \mathrm{~Hz}, 1 \mathrm{H}, 10-\mathrm{H}_{\mathrm{b}}\right), 4.13\left(\mathrm{q},{ }^{3} J_{20,21}=6.6 \mathrm{~Hz}, 1 \mathrm{H}, 20-\mathrm{H}\right), 4.24$ (dd, ${ }^{3} J_{9,10 b}=9.4 \mathrm{~Hz},{ }^{3} J_{9,10 \mathrm{a}}=$ $6.0 \mathrm{~Hz}, 1 \mathrm{H}, 9-\mathrm{H}), 5.08\left(\mathrm{~d},{ }^{2} J_{25 a, 25 \mathrm{~b}}=12.6 \mathrm{~Hz}, 1 \mathrm{H}, 25-\mathrm{H}_{\mathrm{a}}\right), 5.13\left(\mathrm{~d},{ }^{2} J_{25 \mathrm{~b}, 25 \mathrm{a}}=12.6 \mathrm{~Hz}, 1 \mathrm{H}, 25-\mathrm{H}_{\mathrm{b}}\right), 6.38(\mathrm{~m}$, $1 \mathrm{H}, 14-\mathrm{H}$ ), 7.07 (dd, ${ }^{3} J_{18,17}=8.5 \mathrm{~Hz},{ }^{4} J_{18,12}=1.6 \mathrm{~Hz}, 1 \mathrm{H}, 18-\mathrm{H}$ ), 7.18 (ddd, ${ }^{3} J_{4,3}={ }^{3} J_{4,5}=7.5 \mathrm{~Hz},{ }^{4} J_{4,6}=1.6$ $\mathrm{Hz}, 1 \mathrm{H}, 4-\mathrm{H}$ ), 7.24 (ddd, $\left.{ }^{3} J_{5,6}=7.9 \mathrm{~Hz},{ }^{3} J_{5,4}=7.5 \mathrm{~Hz},{ }^{4} J_{5,3}=1.3 \mathrm{~Hz}, 1 \mathrm{H}, 5-\mathrm{H}\right), 7.26(\mathrm{~m}, 1 \mathrm{H}, 15-\mathrm{H}), 7.29-$ $7.38(\mathrm{~m}, 6 \mathrm{H}, 17-\mathrm{H} / 27-\mathrm{H} / 28-\mathrm{H} / 29-\mathrm{H}), 7.45\left(\mathrm{dd},{ }^{3} J_{3,4}=7.5 \mathrm{~Hz},{ }^{4} J_{3,5}=1.3 \mathrm{~Hz}, 1 \mathrm{H}, 3-\mathrm{H}\right), 7.50(\mathrm{~m}, 1 \mathrm{H}, 12-$ H), $7.78\left(\mathrm{dd},{ }^{3} J_{6,5}=7.9 \mathrm{~Hz},{ }^{4} J_{6,4}=1.6 \mathrm{~Hz}, 1 \mathrm{H}, 6-\mathrm{H}\right), 9.30$ (bs, $\left.1 \mathrm{H}, \mathrm{N}-\mathrm{H}_{\mathrm{a}}\right), 10.71$ (bs, $1 \mathrm{H}, \mathrm{N}-\mathrm{H}_{\mathrm{b}}$ ).

${ }^{13} \mathrm{C}-N M R$ (125 MHz, DMSO- $\mathrm{d}_{6}$, 373K): $\delta=16.3$ (q, C-1), 18.0 (q, C-21), 22.8 (q, C-23), 25.5 (q, C-23'), 34.5 (t, C-10), 53.6 (d, C-20), 60.4 (d, C-9), 65.9 (t, C-25), 78.6 (s, C-22), 100.4 (d, C-14), 110.6 (d, C-17), 120.2 (d, C-12), 122.2 (d, C-18), 123.1 (d, C-6), 124.8 (d, C-15), 125.1 (d, C-4), 126.2 (d, C-5), 127.1 (s, C-11), 127.2 (d, C-27), 127.4 (d, C-29), 127.5 (s, C-16), 127.9 (d, C-28), 129.2 (d, C-3), 129.7 (s, C-2), 134.7 (s, C-13), 135.8 (s, C-26), 136.1 (s, C-7), 151.6 (s, C-24), 167.8 (s, C-8), 170.0 (s, C-19).

Optical rotation:

$$
[\alpha]_{D}^{20}=-43.1\left[\mathrm{CHCl}_{3}, \mathrm{c}=1.00\right]
$$

HRMS (Cl) m/z: $[\mathrm{M}+\mathrm{H}]^{+}$Calcd for $\mathrm{C}_{32} \mathrm{H}_{35} \mathrm{~N}_{4} \mathrm{O}_{4} \mathrm{~S}$ 571.2374; Found 571.2366

tert-Butyl 5-((S)-2-((S)-3-((benzyloxy)carbonyl)-2,2,4-trimethyl-5-oxoimidazolidin-1-yl)-3-((2-(methylthio)phenyl)amino)-3-oxopropyl)-1H-indole-1-carboxylate (15I)

According to GP carbamate 13 (70.8 mg, 155 umol, 1.0 eq.), tert-butyl 5-iodo-1H-indole-1-carboxylate (107 mg, $311 \mu \mathrm{mol}, 2.0$ eq.), silver carbonate $(86.0 \mathrm{mg}, 311 \mu \mathrm{mol}, 2.0$ eq.), dibenzyl phosphate $(8.7 \mathrm{mg}$, $31.1 \mu \mathrm{mol}, 20 \mathrm{~mol} \%)$ and $\mathrm{Pd}(\mathrm{OAc})_{2}(3.5 \mathrm{mg}, 15.5 \mu \mathrm{mol}, 10 \mathrm{~mol} \%)$ were reacted at $60^{\circ} \mathrm{C}$ for 2 days. The crude product was purified by column chromatography $\left(\mathrm{SiO}_{2}\right.$, Cyclohexane:EtOAc 100:0 $\rightarrow$ 40:60) and the product $15 \mathrm{I}(65.0 \mathrm{mg}, 96.9 \mu \mathrm{mol}, 62 \%)$ was obtained as a white lyophilisate.

$\mathbf{R}_{\mathbf{f}}(\mathbf{1 5 I})=0.31\left(\mathrm{SiO}_{2}, \mathrm{PE}:\right.$ EtOAc 70:30) 


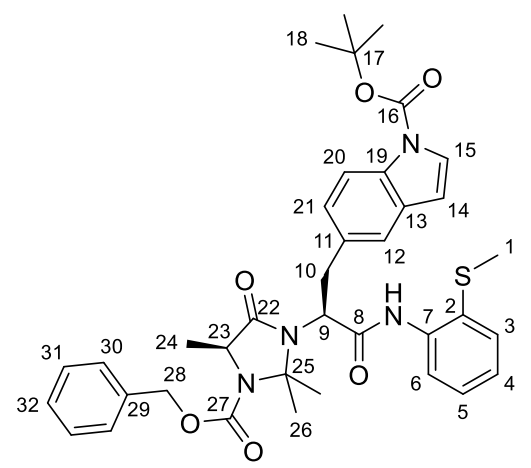

151

${ }^{1} \mathrm{H}-\mathrm{NMR}$ (500 MHz, DMSO-d 6 , 373K): $\delta=1.01(\mathrm{~s}, 3 \mathrm{H}, 26-\mathrm{H}), 1.46\left(\mathrm{~d},{ }^{3} \mathrm{~J}_{24,23}=6.6 \mathrm{~Hz}, 3 \mathrm{H}, 24-\mathrm{H}\right), 1.61$ (s, $\left.3 \mathrm{H}, 26-\mathrm{H}^{\prime}\right), 1.65(\mathrm{~s}, 9 \mathrm{H}, 18-\mathrm{H}), 2.42(\mathrm{~s}, 3 \mathrm{H}, 1-\mathrm{H}), 3.64\left(\mathrm{dd},{ }^{2} J_{10 a, 10 \mathrm{~b}}=13.5 \mathrm{~Hz},{ }^{3} J_{10 a, 9}=6.0 \mathrm{~Hz}, 1 \mathrm{H}, 10-\mathrm{H}_{\mathrm{a}}\right)$, $3.87\left(\mathrm{dd},{ }^{2} J_{10 \mathrm{~b}, 10 \mathrm{a}}=13.5 \mathrm{~Hz},{ }^{3} J_{10 \mathrm{~b}, 9}=9.4 \mathrm{~Hz}, 2 \mathrm{H}, 10-\mathrm{H}_{\mathrm{b}}\right), 4.14\left(\mathrm{q},{ }^{3} J_{23,24}=6.6 \mathrm{~Hz}, 1 \mathrm{H}, 23-\mathrm{H}\right.$ ), 4.30 (dd, $\left.{ }^{3} J_{g, 10 b}=9.4 \mathrm{~Hz},{ }^{3} g_{9,10 a}=6.0 \mathrm{~Hz}, 1 \mathrm{H}, 9-\mathrm{H}\right), 5.08\left(\mathrm{~d},{ }^{2} J_{28 a, 28 b}=12.6 \mathrm{~Hz}, 1 \mathrm{H}, 28-\mathrm{H}_{\mathrm{a}}\right), 5.13\left(\mathrm{~d},{ }^{2} J_{28 \mathrm{~b}, 28 \mathrm{a}}=12.6 \mathrm{~Hz}\right.$, $1 \mathrm{H}, 28-\mathrm{H}_{\mathrm{b}}$ ), 6.65 (d, ${ }^{3} \mathrm{~J}_{14,15}=3.5 \mathrm{~Hz}, 1 \mathrm{H}, 14-\mathrm{H}$ ), 7.19 (ddd, ${ }^{3} \mathrm{~J}_{4,3}=7.9 \mathrm{~Hz},{ }^{3} J_{4,5}=7.5 \mathrm{~Hz},{ }^{4} \mathrm{~J}_{4,6}=1.6 \mathrm{~Hz}, 1 \mathrm{H}$, 4-H), 7.24 (ddd, $\left.{ }^{3} J_{5,6}=7.9 \mathrm{~Hz},{ }^{3} J_{5,4}=7.5 \mathrm{~Hz},{ }^{4} J_{5,3}=1.3 \mathrm{~Hz}, 1 \mathrm{H}, 5-\mathrm{H}\right), 7.29-7.38(\mathrm{~m}, 6 \mathrm{H}, 21-\mathrm{H} / 30-\mathrm{H} / 31-$ $\mathrm{H} / 32-\mathrm{H}), 7.45\left(\mathrm{dd},{ }^{3} J_{3,4}=7.9 \mathrm{~Hz},{ }^{4} J_{3,5}=1.3 \mathrm{~Hz}, 1 \mathrm{H}, 3-\mathrm{H}\right), 7.57\left(\mathrm{~d},{ }^{4} J_{12,21}=1.1 \mathrm{~Hz}, 1 \mathrm{H}, 12-\mathrm{H}\right), 7.61(\mathrm{~d}$, $\left.{ }^{3} J_{15,14}=3.5 \mathrm{~Hz}, 1 \mathrm{H}, 15-\mathrm{H}\right), 7.75\left(\mathrm{dd},{ }^{3} J_{6,5}=7.9 \mathrm{~Hz},{ }^{4} J_{6,4}=1.6 \mathrm{~Hz}, 1 \mathrm{H}, 6-\mathrm{H}\right), 8.00\left(\mathrm{~d},{ }^{3} J_{20,21}=8.5 \mathrm{~Hz}, 1 \mathrm{H}, 20-\right.$ H), 9.27 (bs, $1 \mathrm{H}, \mathrm{N}-\mathrm{H})$.

${ }^{13}$ C-NMR (100 MHz, DMSO-d 6 ): $\delta=15.7$ (q, C-1), 18.9 (q, C-24), 23.0 (q, C-26), 25.2 (q, C-26'), 27.6 (q, C-18), 34.3 (t, C-10), 53.8 (d, C-23), 59.4 (d, C-9), 66.2 (t, C-28), 78.7 (s, C-25), 83.7 (s, C-17), 107.4 (d, C-14), 114.3 (d, C-20), 121.9 (d, C-12), 124.8 (d, C-6), 125.9 (d, C-21), 125.9 (d, C-15), 126.1 (d, C-4), 126.2 (d, C-5), 127.7 (d, C-3), 127.8 (d, C-30), 128.0 (d, C-32), 128.4 (d, C-31), 130.3 (s, C-19), 131.8 (s, C-2), 132.1 (s, C-11), 133.5 (s, C-13), 135.3 (s, C-7), 136.2 (s, C-29), 149.0 (s, C-16), 151.7 (s, C-27), 167.9 (s, C-8), 170.3 (s, C-22).

Optical rotation:

$$
[\alpha]_{D}^{20}=-43.2\left[\mathrm{CHCl}_{3}, \mathrm{c}=1.00\right]
$$

HRMS (Cl) m/z: [M + 2H - Boc] $]^{+}$Calcd for $\mathrm{C}_{32} \mathrm{H}_{35} \mathrm{~N}_{4} \mathrm{O}_{4} \mathrm{~S} 571.2374$; Found 571.2391

\section{Benzyl (S)-2,2,5-trimethyl-3-((S)-1-((2-(methylthio)phenyl)amino)-1-oxo-3-(pyridin-2-yl)propan-2- yl)-4-oxoimidazolidine-1-carboxylate $(15 \mathrm{~m})$}

According to GP carbamate 13 (68.7 mg, $151 \mu \mathrm{mol}, 1.0$ eq.), 2-iodopyridine (61.8 mg, $302 \mu \mathrm{mol}, 2.0$ eq.), silver carbonate ( $83.0 \mathrm{mg}, 302 \mu \mathrm{mol}, 2.0$ eq.), dibenzyl phosphate $(8.4 \mathrm{mg}, 30.2 \mu \mathrm{mol}, 20 \mathrm{~mol} \%)$ and $\mathrm{Pd}(\mathrm{OAc})_{2}(3.4 \mathrm{mg}, 15.1 \mu \mathrm{mol}, 10 \mathrm{~mol} \%)$ were reacted at $60{ }^{\circ} \mathrm{C}$ for 2 days and $80^{\circ} \mathrm{C}$ for 1 day. The

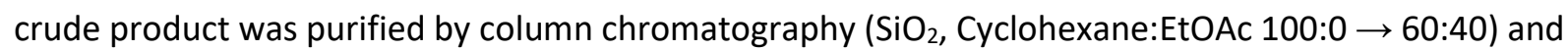
the product $15 \mathrm{~m}$ ( $35.2 \mathrm{mg}, 66.1 \mu \mathrm{mol}, 44 \%)$ was obtained as a white lyophilisate.

$\mathbf{R}_{\mathbf{f}}(\mathbf{1 5 m})=0.11\left(\mathrm{SiO}_{2}\right.$, PE:EtOAc 50:50) 


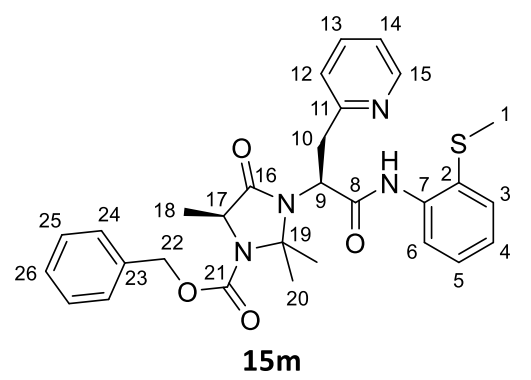

\section{Major rotamer:}

${ }^{1} \mathrm{H}-\mathrm{NMR}\left(400 \mathrm{MHz}\right.$, DMSO-d 6 ): $\delta=1.15(\mathrm{~s}, 3 \mathrm{H}, 20-\mathrm{H}), 1.40\left(\mathrm{~d},{ }^{3} \mathrm{~J}_{18,17}=6.4 \mathrm{~Hz}, 3 \mathrm{H}, 18-\mathrm{H}\right), 1.63(\mathrm{~s}, 3 \mathrm{H}$, $\left.20-\mathrm{H}^{\prime}\right), 2.40(\mathrm{~s}, 3 \mathrm{H}, 1-\mathrm{H}), 3.73\left(\mathrm{dd},{ }^{2} \mathrm{~J}_{10 \mathrm{a}, 10 \mathrm{~b}}=14.1 \mathrm{~Hz},{ }^{3} \mathrm{~J}_{10 \mathrm{a}, 9}=6.7 \mathrm{~Hz}, 1 \mathrm{H}, 10-\mathrm{H}_{\mathrm{a}}\right), 3.77\left(\mathrm{dd},{ }^{2} \mathrm{~J}_{10 \mathrm{~b}, 10 \mathrm{a}}=14.1\right.$ $\left.\mathrm{Hz},{ }^{3} J_{10 \mathrm{~b}, 9}=7.8 \mathrm{~Hz}, 2 \mathrm{H}, 10-\mathrm{H}_{\mathrm{b}}\right), 4.10\left(\mathrm{q},{ }^{3} \mathrm{~J}_{17,18}=6.4 \mathrm{~Hz}, 1 \mathrm{H}, 17-\mathrm{H}\right), 4.58(\mathrm{~m}, 1 \mathrm{H}, 9-\mathrm{H}), 5.06\left(\mathrm{~d},{ }^{2} \mathrm{~J}_{21 \mathrm{a}, 21 \mathrm{~b}}=\right.$ $\left.12.4 \mathrm{~Hz}, 1 \mathrm{H}, 22-\mathrm{H}_{\mathrm{a}}\right), 5.14\left(\mathrm{~d},{ }^{2} \mathrm{~J}_{21 \mathrm{~b}, 21 \mathrm{a}}=12.4 \mathrm{~Hz}, 1 \mathrm{H}, 22-\mathrm{H}_{\mathrm{b}}\right), 7.16-7.24(\mathrm{~m}, 2 \mathrm{H}, 4-\mathrm{H} / 5-\mathrm{H}), 7.24-7.42(\mathrm{~m}$, $8 \mathrm{H}, 3-\mathrm{H} / 12-\mathrm{H} / 14-\mathrm{H} / 24-\mathrm{H} / 25-\mathrm{H} / 26-\mathrm{H}), 7.68\left(\mathrm{dd},{ }^{3} J_{6,5}=7.1 \mathrm{~Hz},{ }^{4} J_{6,4}=1.3 \mathrm{~Hz}, 1 \mathrm{H}, 6-\mathrm{H}\right), 7.74$ (ddd, ${ }^{3} J_{13,12}=$ $\left.{ }^{3} J_{13,14}=7.6 \mathrm{~Hz},{ }^{3} J_{13,15}=1.7 \mathrm{~Hz}, 1 \mathrm{H}, 13-\mathrm{H}\right), 8.56\left(\mathrm{dd},{ }^{3} J_{15,14}=3.6 \mathrm{~Hz},{ }^{3} J_{15,12}=1.7 \mathrm{~Hz}, 1 \mathrm{H}, 15-\mathrm{H}\right), 9.57$ (bs, 1 $\mathrm{H}, \mathrm{N}-\mathrm{H})$.

${ }^{13}$ C-NMR (100 MHz, DMSO-d d $\left._{6}\right): \delta=16.1$ (q, C-1), 18.9 (q, C-18), 23.3 (q, C-20), 25.2 (q, C-20'), 37.6 (t, C-10), 53.7 (d, C-17), 56.5 (d, C-9), 66.2 (t, C-22), 78.8 (s, C-19), 122.0 (d, C-14), 124.0 (d, C-6), 124.2 (d, C-12), 125.8 (d, C-4), 126.2 (d, C-5), 127.7 (d, C-3), 128.0 (d, C-24), 128.5 (d, C-26), 128.5 (d, C-25), 130.8 (s, C-2), 135.8 (s, C-7), 136.3 (s, C-23), 136.8 (d, C-13), 149.0 (d, C-15), 151.8 (s, C-21), 157.6 (s, C-11), 167.7 (s, C-8), 170.0 (s, C-16).

Minor rotamer (selected signals):

${ }^{1} \mathrm{H}-\mathrm{NMR}\left(400 \mathrm{MHz}\right.$, DMSO-d $\left.\mathrm{d}_{6}\right): \delta=1.06(\mathrm{~s}, 3 \mathrm{H}, 20-\mathrm{H}), 4.47(\mathrm{~m}, 1 \mathrm{H}, 9-\mathrm{H}), 9.49$ (bs, $\left.1 \mathrm{H}, \mathrm{N}-\mathrm{H}\right)$.

HRMS (Cl) m/z: [M + H] $]^{+}$Calcd for $\mathrm{C}_{29} \mathrm{H}_{33} \mathrm{~N}_{4} \mathrm{O}_{4} \mathrm{~S}$ 533.2217; Found 533.2230

\section{(S)-2-((S)-3-((Benzyloxy)carbonyl)-2,2,4-trimethyl-5-oxoimidazolidin-1-yl)-3-(p-tolyl)propanoic acid} (16)

\section{Boc-protection:}

$\mathrm{Boc}_{2} \mathrm{O}$ ( $236 \mathrm{mg}, 1.08 \mathrm{mmol}, 4.0 \mathrm{eq}$ ) ) and 4-dimethylaminopyridine ( $6.6 \mathrm{mg}, 54.0 \mu \mathrm{mol}, 20 \mathrm{~mol} \%$ ) were added to a solution of carbamate $15 \mathrm{a}(148 \mathrm{mg}, 270 \mu \mathrm{mol}, 1.0$ eq.) in $\mathrm{MeCN}(3.4 \mathrm{~mL}$ ) and the reaction mixture was stirred at room temperature overnight. The resulting dark solution was filtrated through a short silica column and the filtrate was concentrated to dryness in vacuo to afford the Boc-protected aminoquinoline-derivative, which was used in the next step without further purification.

\section{Saponification:}

The previously prepared derivative was dissolved in THF $(3.4 \mathrm{~mL})$ and cooled with an ice bath. Subsequently, lithium hydroxide monohydrate $(45.5 \mathrm{mg}, 1.08 \mathrm{mmol}, 4.0$ eq.) in water $(1.7 \mathrm{~mL})$ and hydrogen peroxide solution ( $276 \mu \mathrm{L}, 2.70 \mathrm{mmol}, 10$ eq., $30 \mathrm{wt} \%$ ) were added at $0{ }^{\circ} \mathrm{C}$ and the reaction mixture was stirred at room temperature for 6 hours. In the next step, the mixture was diluted with aqueous sodium sulfite solution $(1 \mathrm{M})$, acidified with aqeous $\mathrm{HCl}(1 \mathrm{M})$ and extracted three times with 
ethyl acetate. Afterwards, the combined organics were dried over $\mathrm{Na}_{2} \mathrm{SO}_{4}$ and filtrated. After solvent removal in vacuo, the crude product was purified by column chromatography $\left(\mathrm{SiO}_{2}\right.$, cyclohexane:EtOAc $(2 \% \mathrm{HOAC})$ 100:0 $\rightarrow 50: 50$ ) followed by reverse phase column chromatography ( $\mathrm{C} 18, \mathrm{H}_{2} \mathrm{O}(0.1 \%$ $\mathrm{HCOOH}$ ):MeCN 100:0 $\rightarrow$ 0:100) to afford carboxylic acid 16 (89.7 mg, $211 \mu \mathrm{mol}, 78 \%$ ) as a colorless resin.

$\mathbf{R}_{\mathbf{f}}(\mathbf{1 6})=0.31\left(\mathrm{SiO}_{2}, \mathrm{PE}: \mathrm{EtOAc} 60: 40\right)$

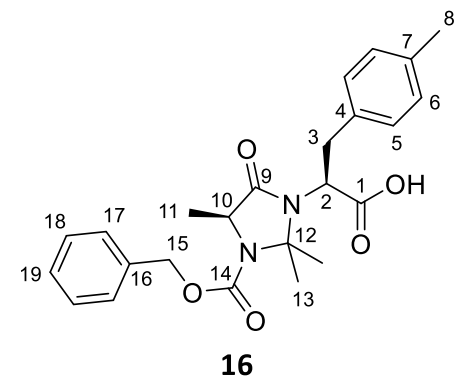

\section{Major rotamer:}

${ }^{1} \mathrm{H}-\mathrm{NMR}\left(400 \mathrm{MHz}, \mathrm{CDCl}_{3}\right): \delta=0.86(\mathrm{~s}, 3 \mathrm{H}, 13-\mathrm{H}), 1.46\left(\mathrm{~d},{ }^{3} \mathrm{~J}_{11,10}=6.6 \mathrm{~Hz}, 3 \mathrm{H}, 11-\mathrm{H}\right), 1.57(\mathrm{~s}, 3 \mathrm{H}, 13-$ $\left.\mathrm{H}^{\prime}\right), 2.30(\mathrm{~s}, 3 \mathrm{H}, 8-\mathrm{H}), 3.45\left(\mathrm{dd},{ }^{2} J_{3 \mathrm{a}, 3 \mathrm{~b}}=13.7 \mathrm{~Hz},{ }^{3} J_{3 \mathrm{a}, 2}=4.7 \mathrm{~Hz}, 1 \mathrm{H}, 3-\mathrm{H}_{\mathrm{a}}\right), 3.69\left(\mathrm{dd},{ }^{2} J_{3 \mathrm{~b}, 3 \mathrm{a}}=13.7 \mathrm{~Hz},{ }^{3} \mathrm{~J}_{3 \mathrm{~b}, 2}=\right.$ $\left.10.6 \mathrm{~Hz}, 1 \mathrm{H}, 3-\mathrm{H}_{\mathrm{b}}\right), 3.83\left(\mathrm{dd}, 3^{3} \mathrm{~J}_{2,3 \mathrm{~b}}=10.6 \mathrm{~Hz}, 3_{2,3 \mathrm{a}}=4.7 \mathrm{~Hz}, 1 \mathrm{H}, 2-\mathrm{H}\right), 4.20\left(\mathrm{q}, 3^{3} J_{10,11}=6.6 \mathrm{~Hz}, 1 \mathrm{H}, 10-\mathrm{H}\right.$ ), $5.06\left(\mathrm{~d},{ }^{2} J_{15 a, 15 b}=12.5 \mathrm{~Hz}, 1 \mathrm{H}, 15-\mathrm{H}_{\mathrm{a}}\right), 5.16\left(\mathrm{~d},{ }^{2} \mathrm{~J}_{15 \mathrm{~b}, 15 \mathrm{a}}=12.5 \mathrm{~Hz}, 1 \mathrm{H}, 15-\mathrm{H}_{\mathrm{b}}\right), 7.05-7.10(\mathrm{sh}, 4 \mathrm{H}, 5-\mathrm{H} / 6-$ H), $7.28-7.40$ (sh, $5 \mathrm{H}, 17-\mathrm{H} / 18-\mathrm{H} / 19-\mathrm{H}), 9.43$ (bs, $1 \mathrm{H}, \mathrm{COO}-\mathrm{H})$.

${ }^{13} \mathrm{C}-N M R\left(100 \mathrm{MHz}, \mathrm{CDCl}_{3}\right.$ ): $\delta=18.7$ (q, C-11), 21.0 (q, C-8), 23.7 (q, C-13), 25.4 (q, C-13'), 34.3 (t, C-3), 54.4 (d, C-10), 57.8 (d, C-2), 67.0 (t, C-15), 79.5 (s, C-12), 128.0 (d, C-17), 128.2 (d, C-19), 128.6 (d, C18), 129.3 (d, C-5), 129.5 (d, C-6), 134.1 (s, C-4), 135.9 (s, C-16), 136.7 (s, C-7), 152.2 (s, C-14), 171.6 (s, C-9), $172.9(s, C-1)$.

\section{Minor rotamer (selected signals):}

${ }^{1} \mathrm{H}-\mathrm{NMR}\left(400 \mathrm{MHz}, \mathrm{CDCl}_{3}\right): \delta=0.71(\mathrm{~s}, 3 \mathrm{H}, 13-\mathrm{H}), 1.48-1.53\left(\mathrm{sh}, 6 \mathrm{H}, 11-\mathrm{H} / 13-\mathrm{H}^{\prime}\right), 4.28\left(\mathrm{q},{ }^{3} \mathrm{~J}_{10,11}=6.1\right.$ $\mathrm{Hz}, 1 \mathrm{H}, 10-\mathrm{H})$.

${ }^{13} \mathrm{C}-\mathrm{NMR}\left(100 \mathrm{MHz}, \mathrm{CDCl}_{3}\right): \delta=17.3(\mathrm{q}, \mathrm{C}-11), 24.8(\mathrm{q}, \mathrm{C}-13), 26.5$ (q, C-13'), 54.9 (d, C-10), 67.6 (t, C15).

Optical rotation: $\quad[\alpha]_{D}^{20}=-94\left[\mathrm{CHCl}_{3}, \mathrm{c}=0.50\right]$

HRMS (Cl) m/z: [M + H] $]^{+}$Calcd for $\mathrm{C}_{24} \mathrm{H}_{29} \mathrm{~N}_{2} \mathrm{O}_{5}$ 425.2071; Found 425.2047

Benzyl (S)-3-((S)-1-((2-methoxy-2-oxoethyl)amino)-1-oxo-3-(p-tolyl)propan-2-yl)-2,2,5-trimethyl-4oxoimidazolidine-1-carboxylate (17)

DIPEA (59.6 $\mu \mathrm{L}, 44.1 \mathrm{mg}, 341 \mu \mathrm{mol}, 2.4$ eq.) and TBTU ( $48.0 \mathrm{mg}, 149 \mu \mathrm{mol}, 1.05$ eq.) were added to a solution of carboxylic acid 16 (60.4 mg, $142 \mu \mathrm{mol}, 1.0$ eq.) and methyl glycinate hydrochloride (21.4 $\mathrm{mg}, 171 \mu \mathrm{mol}, 1.2$ eq.) in $\mathrm{MeCN}(1.4 \mathrm{~mL})$ at $0{ }^{\circ} \mathrm{C}$. The reaction mixture was stirred at $0{ }^{\circ} \mathrm{C}$ for 2 hours before it was hydrolyzed with aqueous $\mathrm{HCl}$ solution(1M). Subsequently, the mixture was extracted 
three times with ethyl acetate and the combined organic phases were washed with saturated $\mathrm{NaHCO}_{3}$ solution. In the next step, the organic phase was dried over $\mathrm{Na}_{2} \mathrm{SO}_{4}$ and filtrated. After solvent removal in vacuo, the crude product was purified by column chromatography $\left(\mathrm{C} 18, \mathrm{H}_{2} \mathrm{O}: \mathrm{MeCN}\right.$ 100:0 $\rightarrow$ 0:100) and the product $17(65.9 \mathrm{mg}, 133 \mu \mathrm{mol}, 93 \%)$ was obtained as a white lyophilisate.

$\mathbf{R}_{\mathbf{f}}(\mathbf{1 7})=0.32\left(\mathrm{SiO}_{2}, \mathrm{PE}: \mathrm{EtOAc} 50: 50\right)$

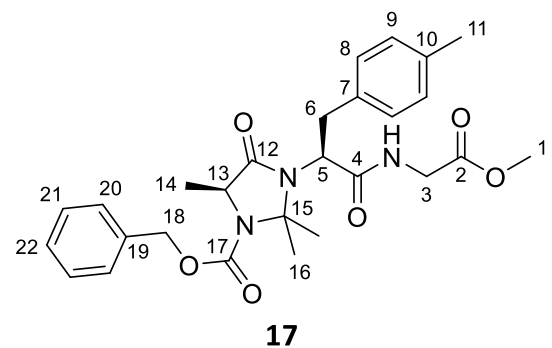

\section{Major rotamer:}

${ }^{1} \mathrm{H}-\mathrm{NMR}\left(400 \mathrm{MHz}, \mathrm{CDCl}_{3}\right): \delta=0.89(\mathrm{~s}, 3 \mathrm{H}, 16-\mathrm{H}), 1.51\left(\mathrm{~d},{ }^{3} \mathrm{~J}_{14,13}=6.2 \mathrm{~Hz}, 3 \mathrm{H}, 14-\mathrm{H}\right), 1.59(\mathrm{~s}, 3 \mathrm{H}, 16-$ $\left.\mathrm{H}^{\prime}\right), 2.30(\mathrm{~s}, 3 \mathrm{H}, 11-\mathrm{H}), 3.19\left(\mathrm{dd},{ }^{2} \mathrm{~J}_{6 \mathrm{a}, 6 \mathrm{~b}}=12.7 \mathrm{~Hz},{ }^{3} \mathrm{~J}_{6 \mathrm{a}, 5}=2.9 \mathrm{~Hz}, 1 \mathrm{H}, 6-\mathrm{H}_{\mathrm{a}}\right), 3.72(\mathrm{~s}, 3 \mathrm{H}, 1-\mathrm{H}), 3.79(\mathrm{~m}, 1$ $\mathrm{H}, 5-\mathrm{H}), 3.83\left(\mathrm{dd},{ }^{2} J_{3 \mathrm{a}, 3 \mathrm{~b}}=18.0 \mathrm{~Hz},{ }^{3} \mathrm{~J}_{3 \mathrm{a}, \mathrm{NH}}=4.7 \mathrm{~Hz}, 1 \mathrm{H}, 3-\mathrm{H}_{\mathrm{a}}\right), 3.90\left(\mathrm{~m}, 1 \mathrm{H}, 6-\mathrm{H}_{\mathrm{b}}\right), 4.18\left(\mathrm{q},{ }^{3} \mathrm{~J}_{13,14}=6.2 \mathrm{~Hz}\right.$, $1 \mathrm{H}, 13-\mathrm{H}$ ), $4.26\left(\mathrm{dd},{ }^{2} J_{3 \mathrm{~b}, 3 \mathrm{a}}=18.0 \mathrm{~Hz},{ }^{3} J_{3 \mathrm{~b}, \mathrm{NH}}=7.1 \mathrm{~Hz}, 1 \mathrm{H}, 3-\mathrm{H}_{\mathrm{b}}\right), 5.05\left(\mathrm{~d},{ }^{2} J_{18 \mathrm{a}, 18 \mathrm{~b}}=12.4 \mathrm{~Hz}, 1 \mathrm{H}, 18-\mathrm{H}_{\mathrm{a}}\right.$ ), $5.15\left(\mathrm{~d},{ }^{2} J_{18 b, 18 \mathrm{a}}=12.4 \mathrm{~Hz}, 1 \mathrm{H}, 18-\mathrm{H}_{\mathrm{b}}\right.$ ), $7.05-7.12$ (sh, $\left.4 \mathrm{H}, 8-\mathrm{H} / 9-\mathrm{H}\right), 7.26-7.40$ (sh, $5 \mathrm{H}, 20-\mathrm{H} / 21-\mathrm{H} / 22-$ $\mathrm{H}), 8.10(\mathrm{~m}, 1 \mathrm{H}, \mathrm{N}-\mathrm{H})$.

${ }^{13} \mathrm{C}-\mathrm{NMR}\left(100 \mathrm{MHz}, \mathrm{CDCl}_{3}\right.$ ): $\delta=19.2$ (q, C-14), 21.0 (q, C-11), 22.7 (q, C-16), 26.0 (q, C-16'), 34.4 (t, C6), 41.0 (t, C-3), 52.2 (q, C-1), 54.6 (d, C-13), 62.3 (d, C-5), 67.0 (t, C-18), 80.3 (s, C-15), 128.1 (d, C-20), 128.3 (d, C-22), 128.6 (d, C-21), 129.2 (d, C-8), 129.3 (d, C-9), 133.6 (s, C-7), 135.9 (s, C-19), 136.7 (s, C10), 152.1 (s, C-17), 169.8 (s, C-2), 171.9 (s, C-4), 172.5 (s, C-12).

Minor rotamer (selected signals):

${ }^{1} \mathrm{H}-\mathrm{NMR}\left(400 \mathrm{MHz}, \mathrm{CDCl}_{3}\right): \delta=0.74(\mathrm{~s}, 3 \mathrm{H}, 16-\mathrm{H}), 1.56(\mathrm{~m}, 3 \mathrm{H}, 14-\mathrm{H}), 1.69\left(\mathrm{~s}, 3 \mathrm{H}, 16-\mathrm{H}^{\prime}\right), 4.25(\mathrm{~m}, 1 \mathrm{H}$, $13-\mathrm{H}), 8.15(\mathrm{~m}, 1 \mathrm{H}, \mathrm{N}-\mathrm{H})$.

${ }^{13} \mathrm{C}-\mathrm{NMR}\left(100 \mathrm{MHz}, \mathrm{CDCl}_{3}\right.$ ): $\delta=17.7$ (q, C-14), 23.9 (q, C-16), 27.0 (q, C-16'), 55.1 (d, C-13), 62.5 (d, C5), $67.6(\mathrm{t}, \mathrm{C}-18), 79.8(\mathrm{~s}, \mathrm{C}-15)$.

Optical rotation: $\quad[\alpha]_{D}^{20}=-8\left[\mathrm{CHCl}_{3}, \mathrm{c}=0.50\right]$

HRMS (Cl) m/z: [M + H] ${ }^{+}$Calcd for $\mathrm{C}_{31} \mathrm{H}_{42} \mathrm{~N}_{4} \mathrm{O}_{8} 496.2442$; Found 496.2437

Methyl ((S)-2-((S)-2-((S)-3-(benzyloxy)-2-((tert-butoxycarbonyl)amino)propanamido)propanamido)3-(p-tolyl)propanoyl)glycinate (18)

\section{Hydrogenation:}

Aqueous $\mathrm{HCl}$ solution $(6 \mathrm{M}, 249 \mu \mathrm{L})$ and $\mathrm{Pd} / \mathrm{C}(8.0 \mathrm{mg}$, dry, $10 \mathrm{wt} \% \mathrm{Pd})$ were added to a solution of 17 (74.0 mg, $149 \mu \mathrm{mol}, 1.0$ eq.) in $\mathrm{MeOH}(1.5 \mathrm{~mL}$ ) and the suspension was stirred under hydrogen atmosphere (1 atm) for 1 hour. Subsequently, the catalyst was removed by filtration through a syringe 
filter and the solvent thereafter removed in vacuo to afford the free amine as a yellowish resin, which was used in the next step without further purification.

\section{Aminal cleavage:}

The previously prepared amine was dissolved in a mixture of $\mathrm{MeOH}(750 \mu \mathrm{L})$ and TFA $(750 \mu \mathrm{L})$ and the resulting solution was stirred in a sealed tube at $50{ }^{\circ} \mathrm{C}$ in a heating block overnight. The solvent was removed in vacuo and residual TFA thereafter removed as azeotrope with chloroform to afford the tripeptide as a yellowish resin, which was used in the next step without further purification.

\section{Peptide coupling:}

DIPEA (65.1 $\mu \mathrm{L}, 48.1 \mathrm{mg}, 373 \mu \mathrm{mol}, 2.5$ eq.) and TBTU (52.6 mg, $164 \mu \mathrm{mol}, 1.1$ eq.) were added to a solution of the previously prepared tripeptide and Boc-L-(OBn)Ser-OH (48.4 mg, $164 \mu \mathrm{mol}, 1.1 \mathrm{eq}$.) in $\mathrm{DMF}(1.5 \mathrm{~mL})$ at $0{ }^{\circ} \mathrm{C}$. The reaction mixture was warmed to room temperature overnight before it was hydrolyzed with saturated $\mathrm{NaHCO}_{3}$ solution. Subsequently, the mixture was extracted twice with ethyl acetate and the combined organics were dried over $\mathrm{Na}_{2} \mathrm{SO}_{4}$. After filtration, the solvent was evaporated and the crude product was purified by column chromatography (C18, $\mathrm{H}_{2} \mathrm{O}: \mathrm{MeCN}$ 100:0 $\rightarrow$ 0:100) to afford tetrapeptide $\mathbf{1 8}(71.7 \mathrm{mg}, 120 \mu \mathrm{mol}, 80 \%$ over 3 steps) as a yellowish resin.

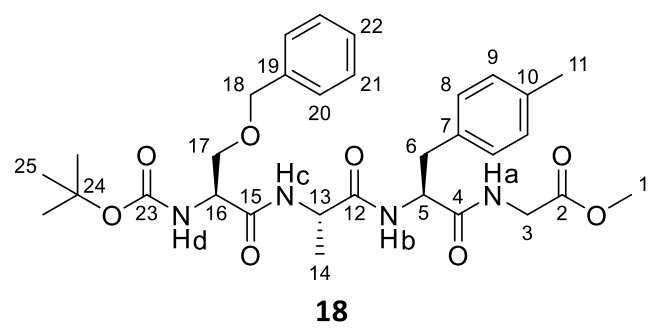

${ }^{1} \mathrm{H}-\mathrm{NMR}\left(400 \mathrm{MHz}, \mathrm{CDCl}_{3}\right): \delta=1.25\left(\mathrm{~d},{ }^{3} \mathrm{~J}_{14,13}=7.1 \mathrm{~Hz}, 3 \mathrm{H}, 14-\mathrm{H}\right), 1.44(\mathrm{~s}, 9 \mathrm{H}, 25-\mathrm{H}), 2.28(\mathrm{~s}, 3 \mathrm{H}, 11-\mathrm{H})$, $2.93\left(\mathrm{dd},{ }^{2} \mathrm{~J}_{6 \mathrm{a}, 6 \mathrm{~b}}=14.3 \mathrm{~Hz},{ }^{3} J_{6 \mathrm{a}, 5}=8.6 \mathrm{~Hz}, 1 \mathrm{H}, 6-\mathrm{H}_{\mathrm{a}}\right), 3.22\left(\mathrm{dd},{ }^{2} \mathrm{~J}_{6 \mathrm{~b}, 6 \mathrm{a}}=14.3 \mathrm{~Hz},{ }^{3} J_{6 b, 5}=5.3 \mathrm{~Hz}, 1 \mathrm{H}, 6-\mathrm{H}_{\mathrm{b}}\right)$, $3.55\left(\mathrm{dd},{ }^{2} \mathrm{~J}_{6 \mathrm{a}, 5}=9.5 \mathrm{~Hz},{ }^{3} \mathrm{~J}_{6 \mathrm{a}, 5}=6.5 \mathrm{~Hz}, 1 \mathrm{H}, 17-\mathrm{H}_{\mathrm{a}}\right), 3.73(\mathrm{~s}, 3 \mathrm{H}, 1-\mathrm{H}), 3.82\left(\mathrm{dd},{ }^{2} \mathrm{~J}_{6 \mathrm{a}, 5}=9.5 \mathrm{~Hz},{ }^{3} \mathrm{~J}_{6 \mathrm{a}, 5}=4.4\right.$ $\left.\mathrm{Hz}, 1 \mathrm{H}, 17-\mathrm{H}_{\mathrm{b}}\right), 3.87\left(\mathrm{dd},{ }^{2} \mathrm{~J}_{3 \mathrm{a}, 3 \mathrm{~b}}=18.1 \mathrm{~Hz},{ }^{3} J_{3 \mathrm{a}, \mathrm{NHa}}=4.8 \mathrm{~Hz}, 1 \mathrm{H}, 3-\mathrm{H}_{\mathrm{a}}\right), 4.14\left(\mathrm{dd},{ }^{2} \mathrm{~J}_{3 \mathrm{~b}, 3 \mathrm{a}}=18.1 \mathrm{~Hz},{ }^{3} J_{3 b, \mathrm{NHa}}=\right.$ $\left.6.1 \mathrm{~Hz}, 1 \mathrm{H}, 3-\mathrm{H}_{\mathrm{b}}\right), 4.25(\mathrm{~m}, 1 \mathrm{H}, 16-\mathrm{H}), 4.30\left(\mathrm{dq},{ }^{3} J_{13,14}={ }^{3} J_{13, \mathrm{NHc}}=7.1 \mathrm{~Hz}, 1 \mathrm{H}, 13-\mathrm{H}\right), 4.52\left(\mathrm{~d},{ }^{2} J_{18 \mathrm{a}, 18 \mathrm{~b}}=\right.$ $\left.12.2 \mathrm{~Hz}, 1 \mathrm{H}, 18-\mathrm{H}_{\mathrm{a}}\right), 4.55\left(\mathrm{~d},{ }^{2} \mathrm{~J}_{18 \mathrm{~b}, 18 \mathrm{a}}=12.2 \mathrm{~Hz}, 1 \mathrm{H}, 18-\mathrm{H}_{\mathrm{b}}\right), 4.71$ (ddd, ${ }^{3} J_{5,6 \mathrm{a}}={ }^{3} J_{5, \mathrm{NHb}}=8.6 \mathrm{~Hz},{ }^{3} J_{5,6 \mathrm{~b}}=5.3$ $\mathrm{Hz}, 1 \mathrm{H}, 5-\mathrm{H}$ ), $5.39\left(\mathrm{~d},{ }^{3} \mathrm{~J}_{\mathrm{NHd}, 16}=5.0 \mathrm{~Hz}, 1 \mathrm{H}, \mathrm{N}-\mathrm{H}_{\mathrm{d}}\right.$ ), $6.76-6.83$ (sh, $3 \mathrm{H}, \mathrm{N}-\mathrm{H}_{\mathrm{a}} / \mathrm{N}-\mathrm{H}_{\mathrm{b}} / \mathrm{N}-\mathrm{H}_{\mathrm{c}}$ ), $7.04-7.06$ (sh, $4 \mathrm{H}, 8-\mathrm{H} / 9-\mathrm{H}), 7.26-7.38$ (sh, $5 \mathrm{H}, 20-\mathrm{H} / 21-\mathrm{H} / 22-\mathrm{H})$.

${ }^{13} \mathrm{C}-N M R(100 \mathrm{MHz}, \mathrm{CDCl}$ ) : $\delta=17.4$ (q, C-14), 21.0 (q, C-11), 28.3 (q, C-25), 36.8 (t, C-6), 41.1 (t, C-3), 50.0 (d, C-13), 52.2 (q, C-1), 53.9 (d, C-5), 54.3 (d, C-16), 69.4 (t, C-17), 73.6 (t, C-18), 80.7 (s, C-24), 127.8 (d, C-20), 128.1 (d, C-22), 128.6 (d, C-21), 128.9 (d, C-8), 129.2 (d, C-9), 133.7 (s, C-7), 136.3 (s, C10), 137.1 (s, C-19), 155.8 (s, C-23), 170.0 (s, C-2), 171.0 (s, C-15), 171.1 (s, C-4), 171.8 (s, C-12).

Optical rotation:

$$
[\alpha]_{D}^{20}=-20\left[\mathrm{CHCl}_{3}, \mathrm{c}=0.50\right]
$$

HRMS (Cl) m/z: $[\mathrm{M}+\mathrm{H}]^{+}$Calcd for $\mathrm{C}_{31} \mathrm{H}_{42} \mathrm{~N}_{4} \mathrm{O}_{8}$ 599.3075; Found 599.3090 


\section{NMR spectra}

Benzyl (S)-2-(((S)-1-oxo-1-(quinolin-8-ylamino)propan-2-yl)carbamoyl)pyrrolidine-1-carboxylate (2) $500 \mathrm{MHz}$, DMSO-d6

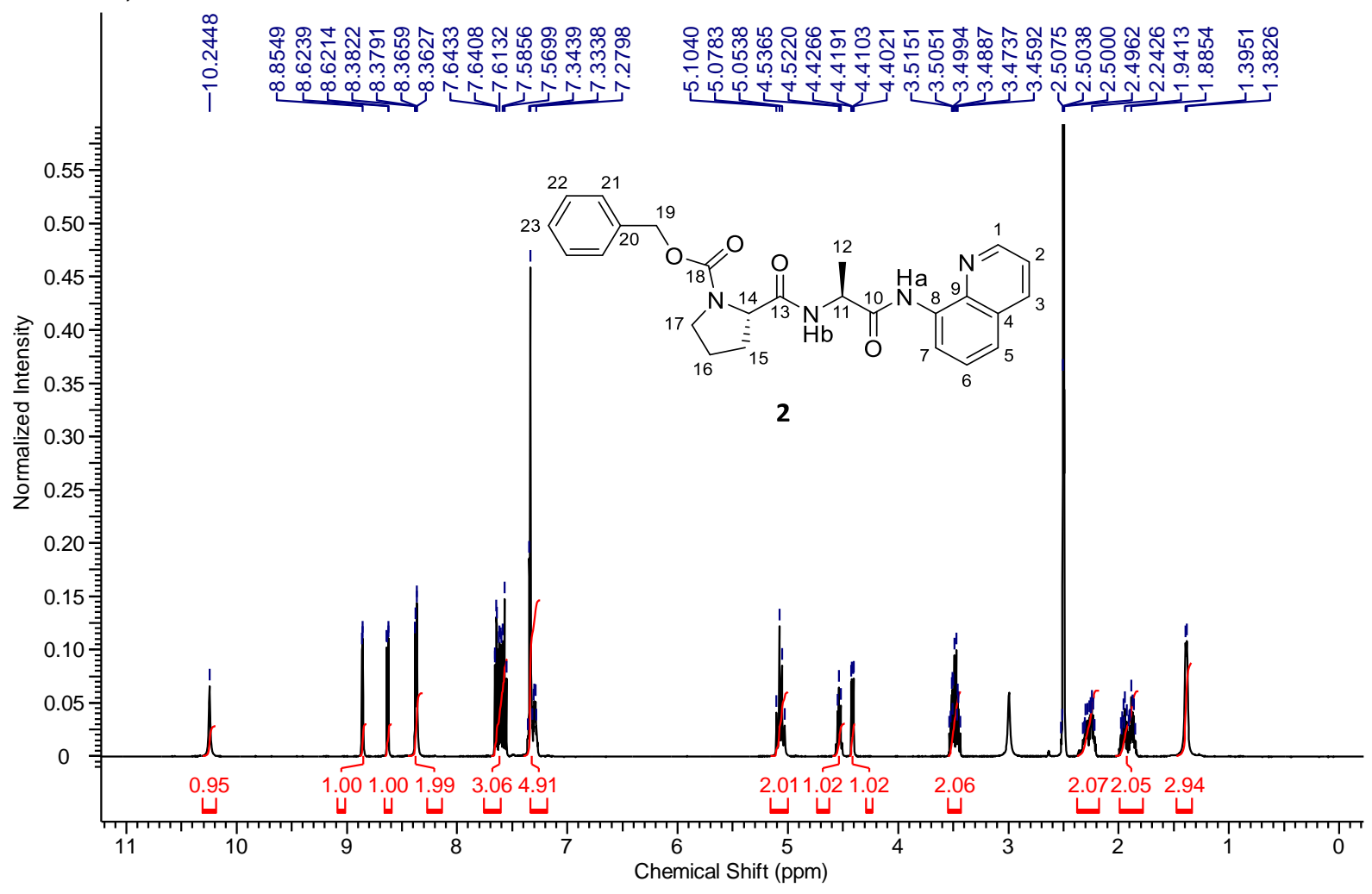

$125 \mathrm{MHz}$, DMSO-d6

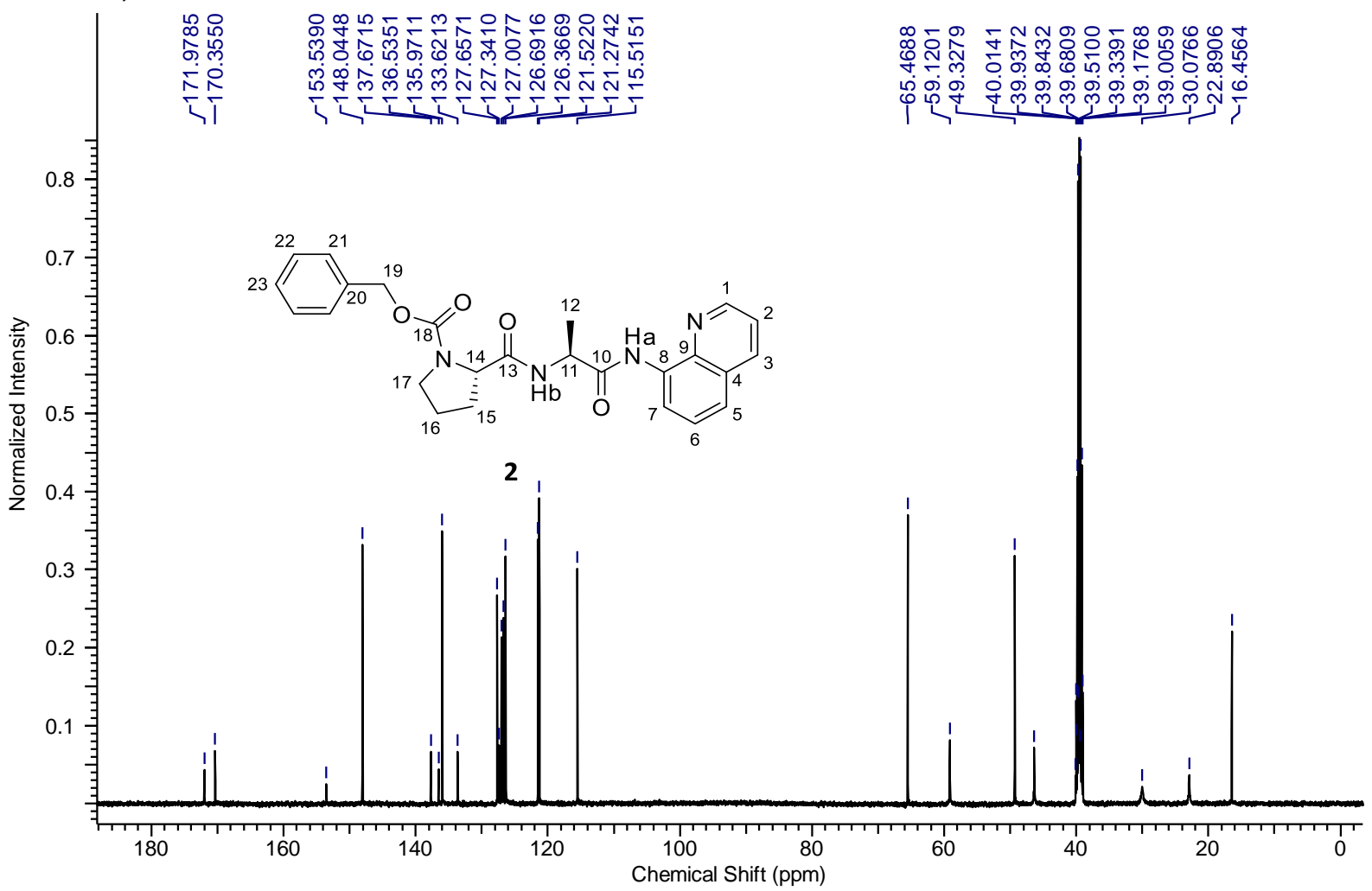


(S)-2-((S)-1-Oxotetrahydro-1H-pyrrolo[1,2-c]imidazol-2(3H)-yl)-N-(quinolin-8-yl)propenamide (3)
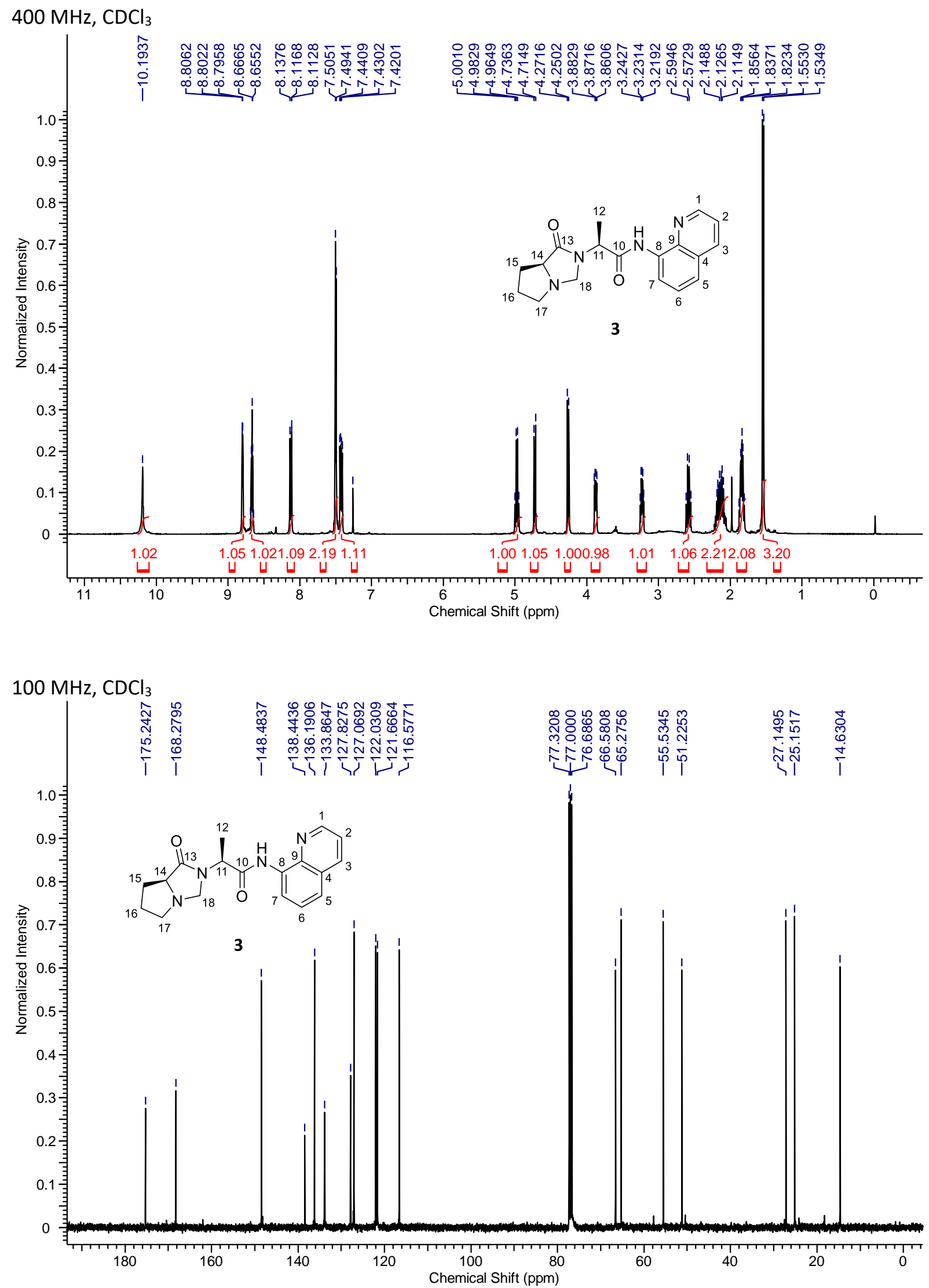
Benzyl ((S)-1-oxo-1-(((S)-1-oxo-1-(quinolin-8-ylamino)propan-2-yl)amino)propan-2-yl)carbamate (4)

$400 \mathrm{MHz}, \mathrm{CDCl}_{3}$

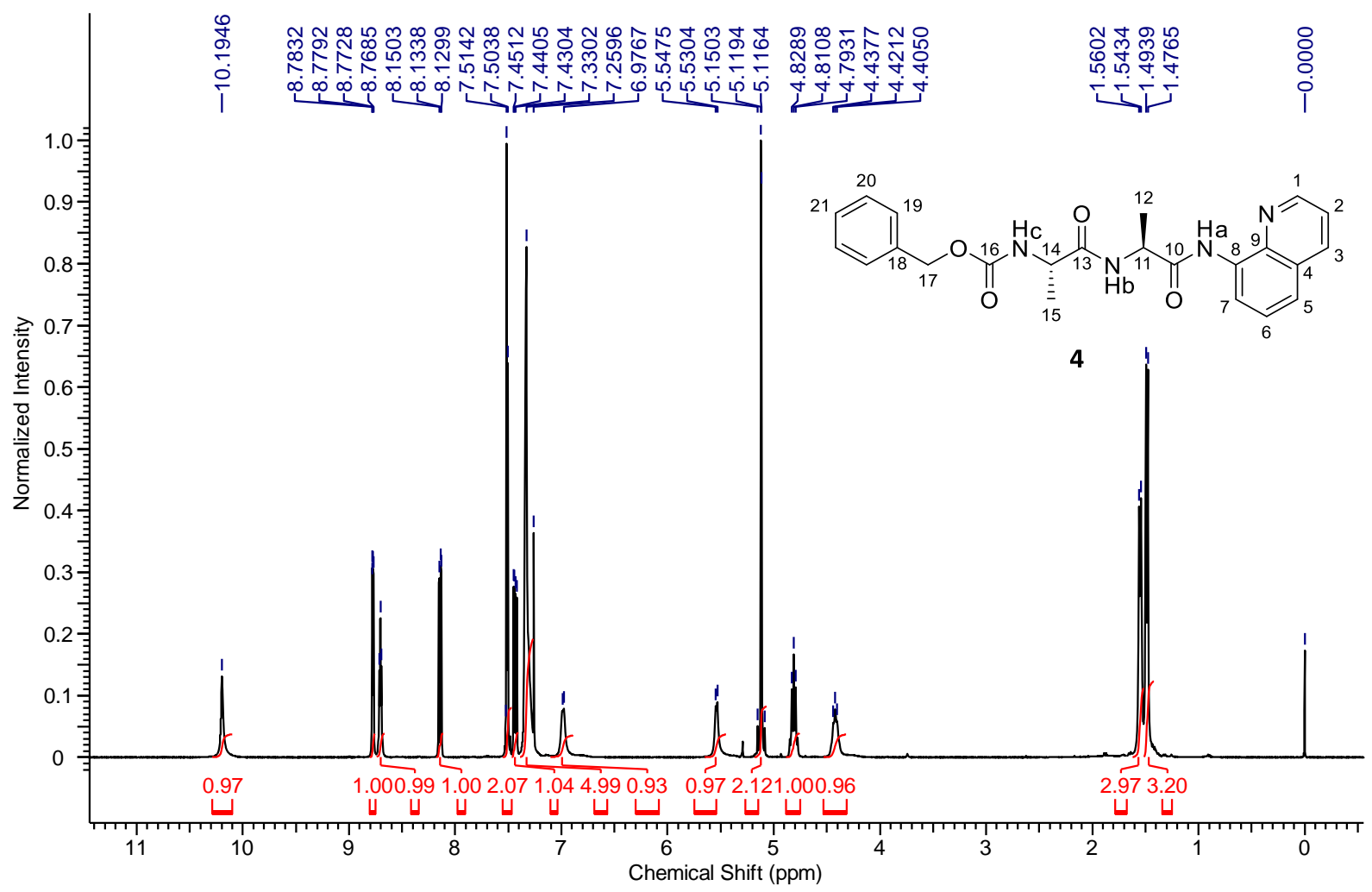

$100 \mathrm{MHz}, \mathrm{CDCl}_{3}$

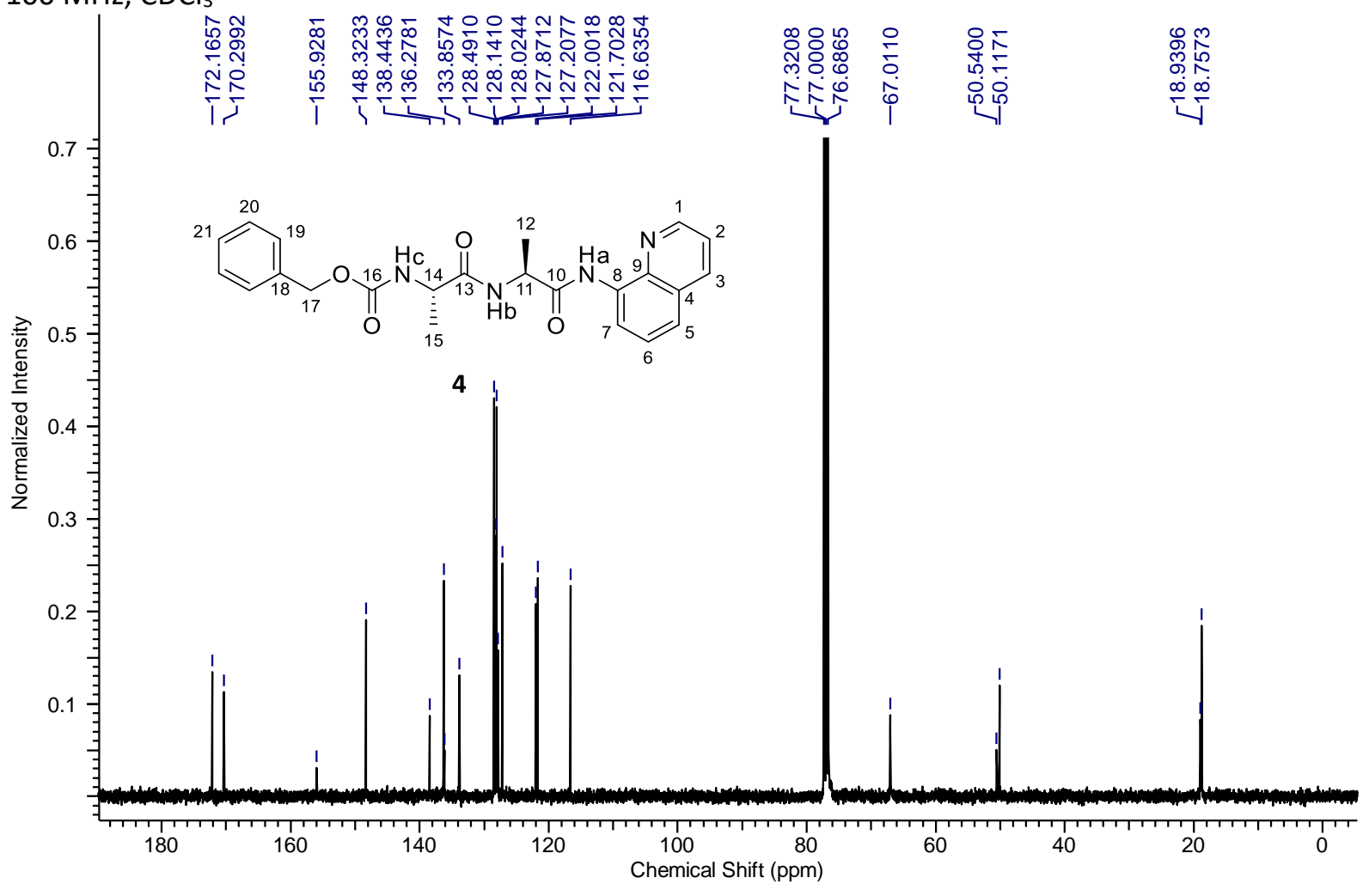


Benzyl (S)-5-methyl-4-oxo-3-((S)-1-oxo-1-(quinolin-8-ylamino)propan-2-yl)imidazoledine-1-carboxylate (5)

$500 \mathrm{MHz}$, DMSO-d6

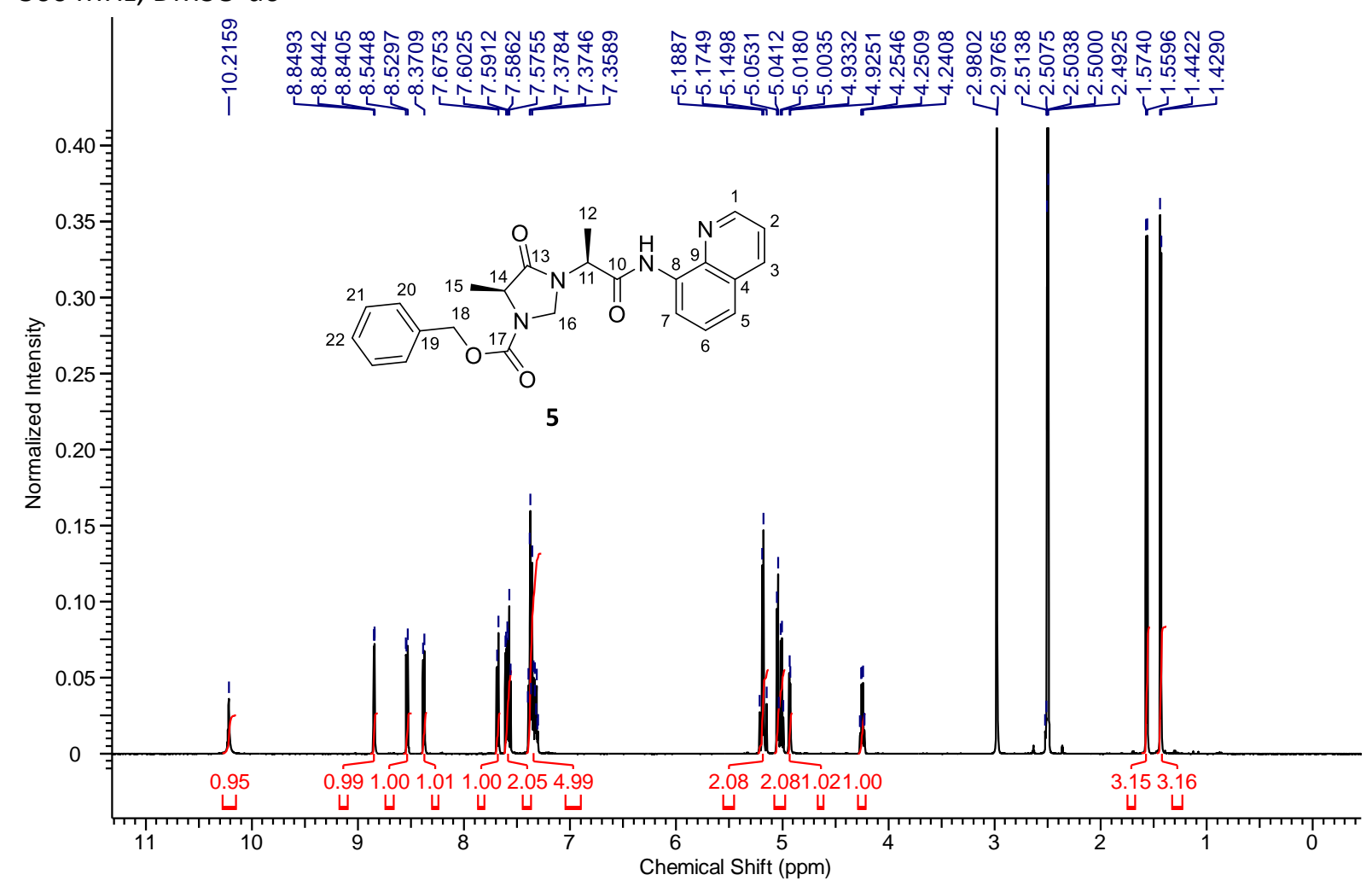

$125 \mathrm{MHz}$, DMSO-d6

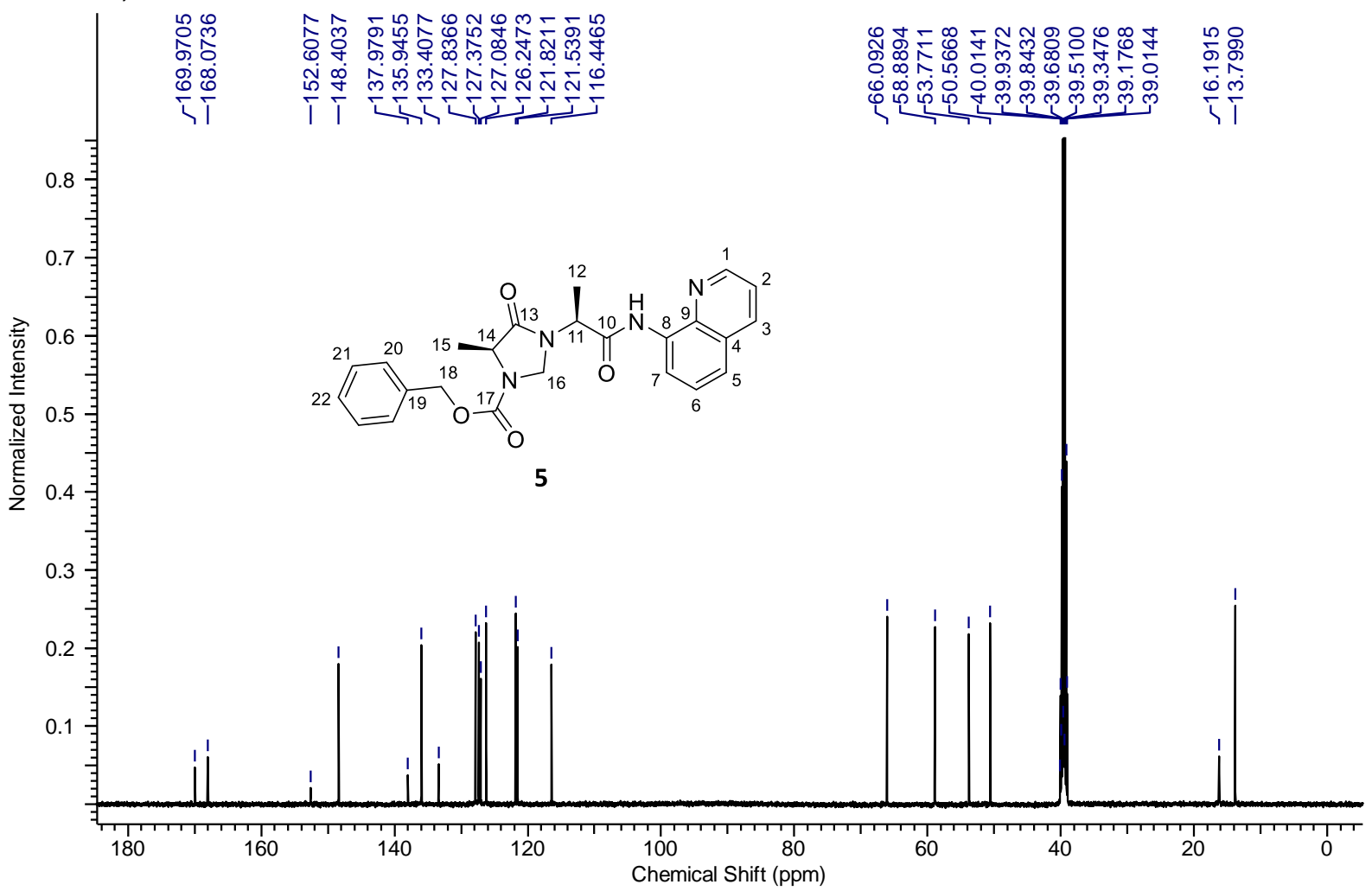


Benzyl (S)-5-methyl-4-oxo-3-((S)-1-oxo-1-(quinolin-8-ylamino)-3-( $p$-tolyl)propan-2-yl)imidazolidine1-carboxylate (6)

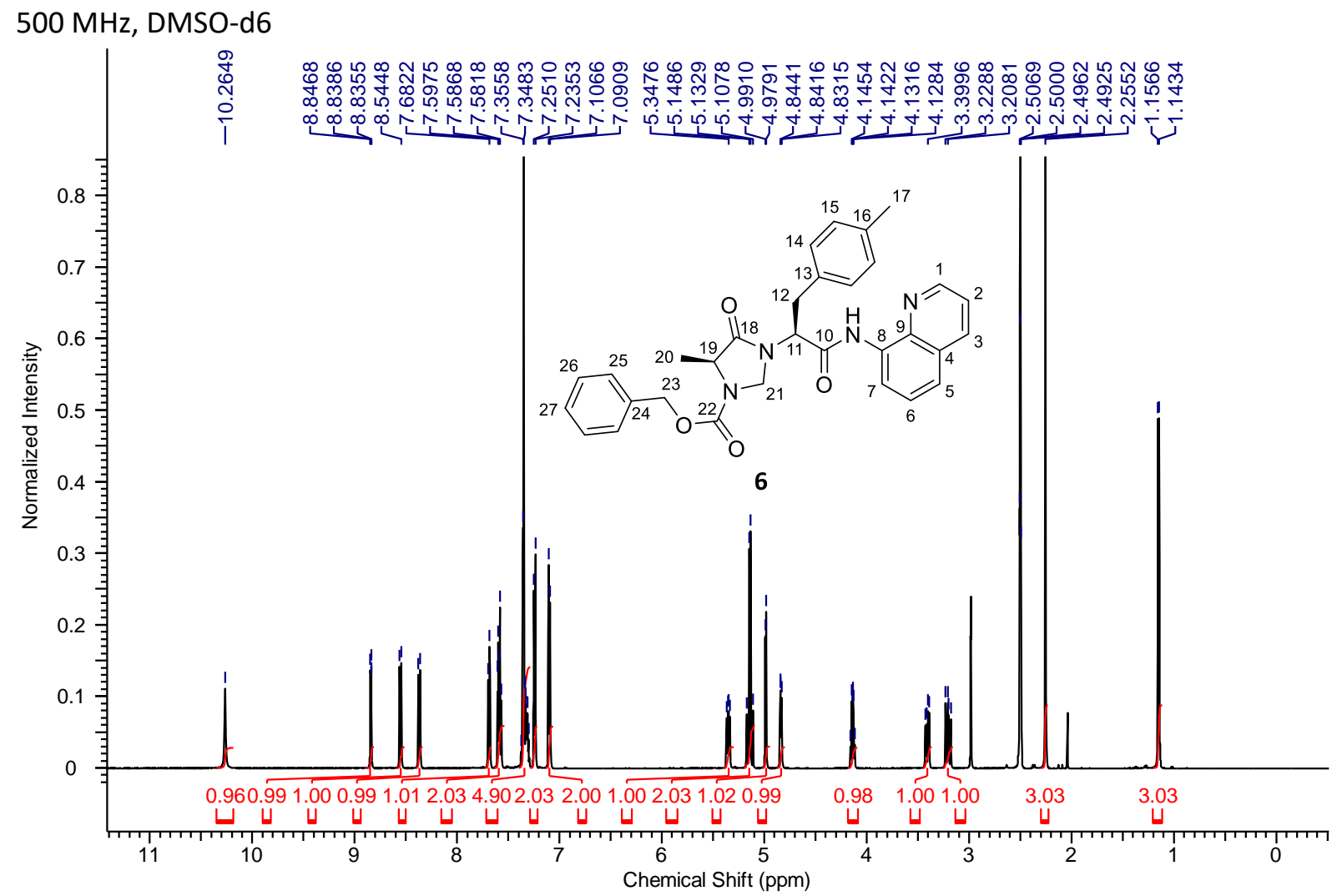

$125 \mathrm{MHz}$, DMSO-d6

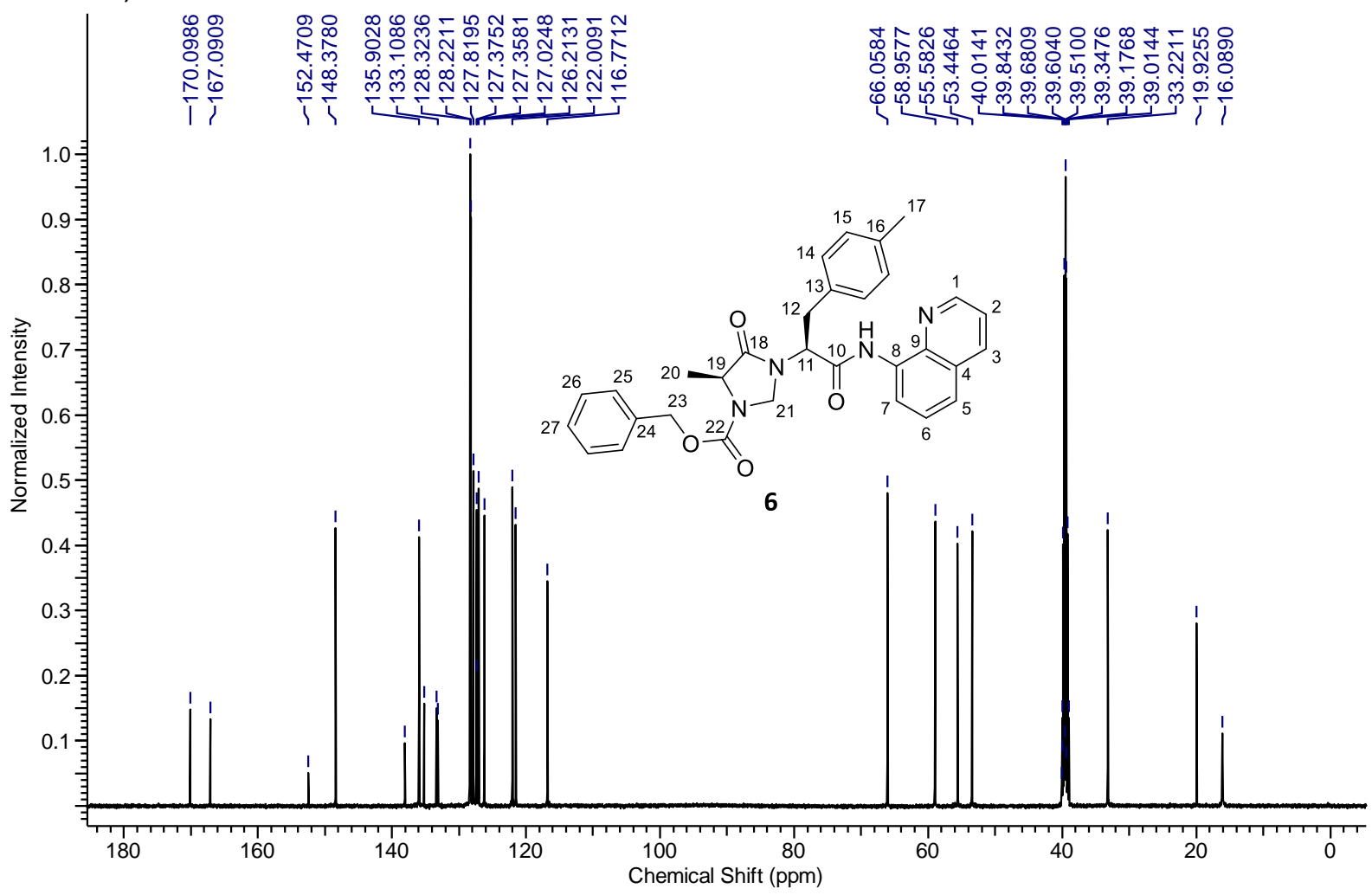


(S)-2-((S)-4-Methyl-5-oxoimidazolidin-1-yl)-N-(quinolin-8-yl)propenamide (7)

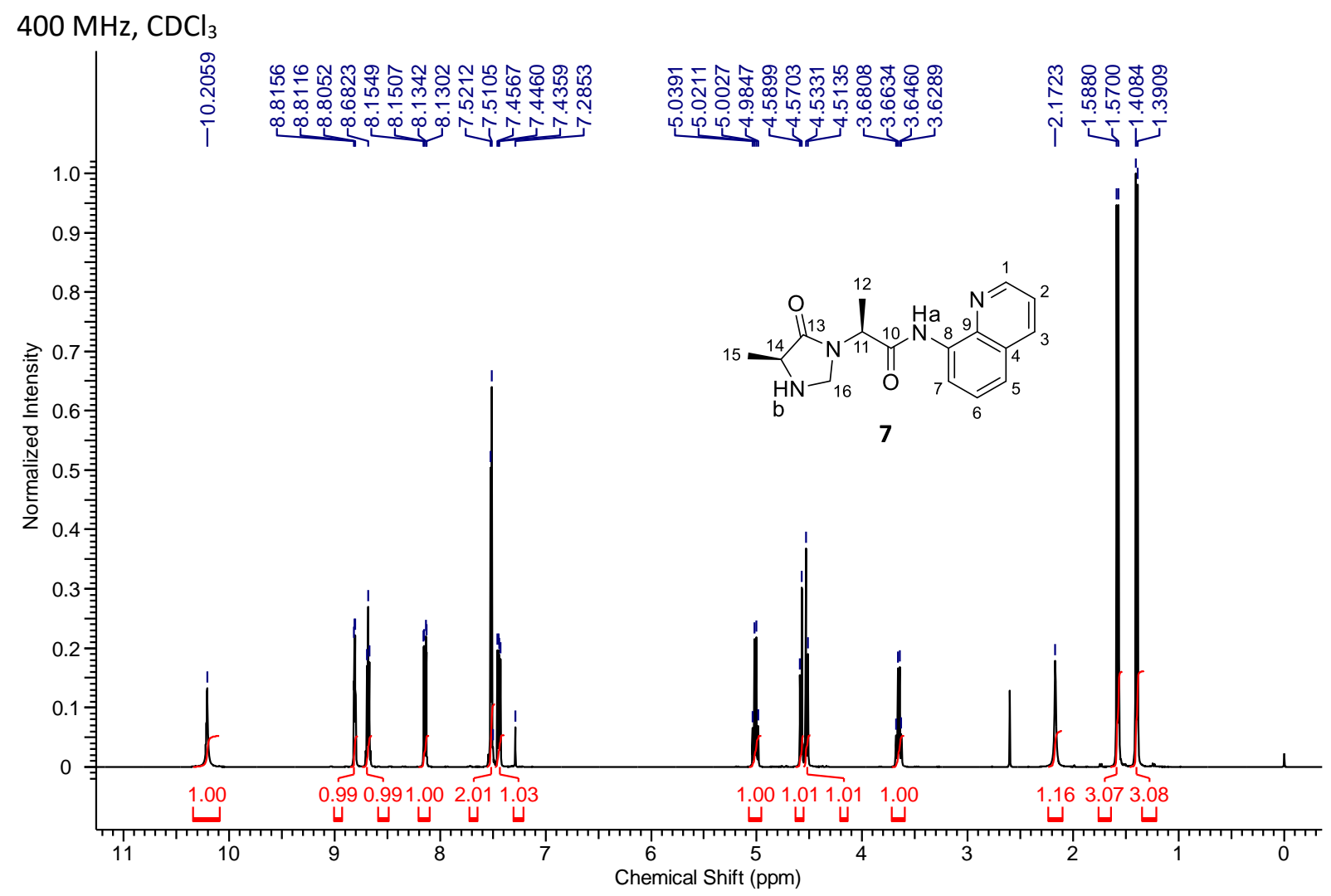

$100 \mathrm{MHz}, \mathrm{CDCl}_{3}$

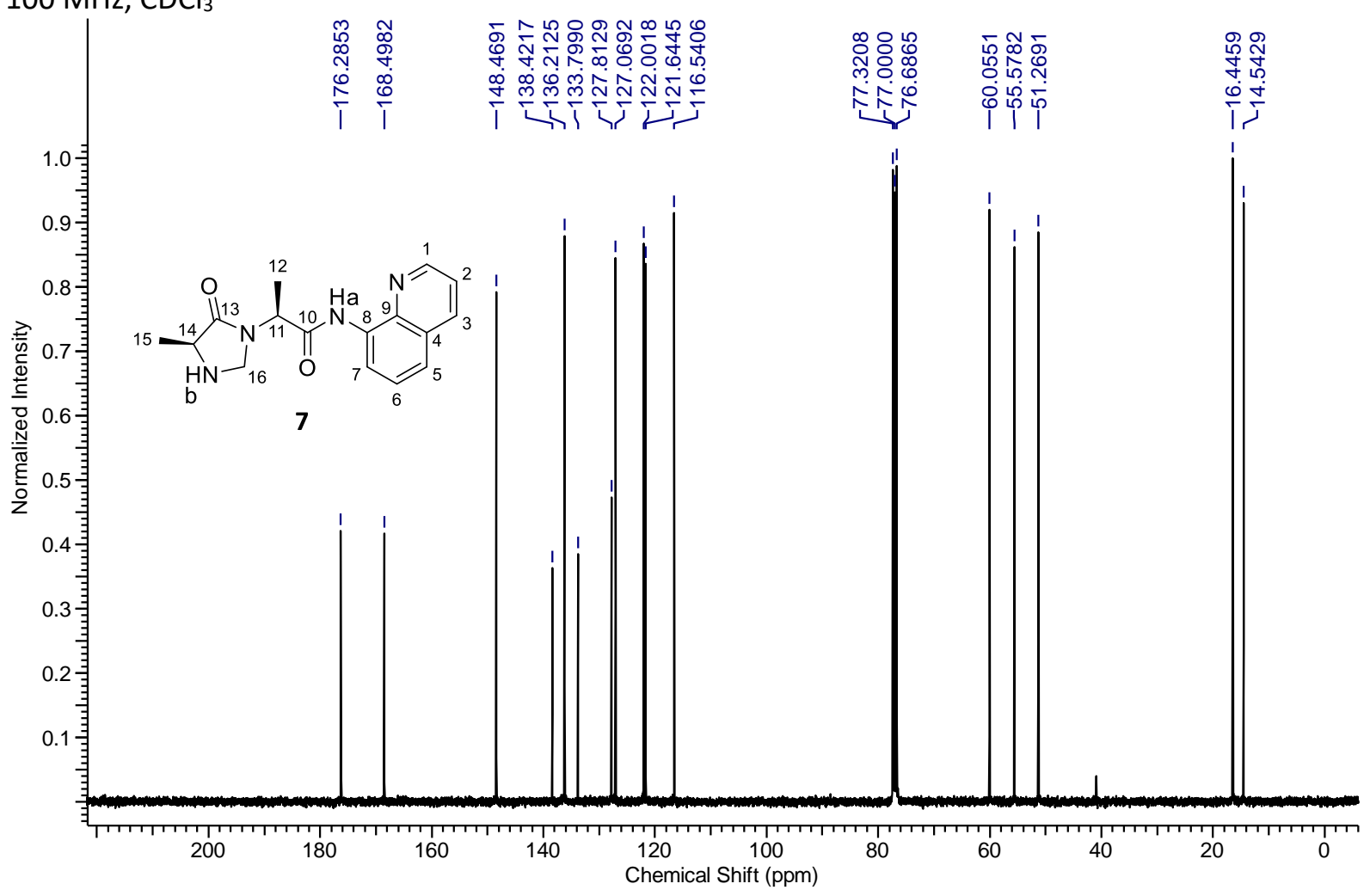


(S)-N-(Quinolin-8-yl)-2-((S)-2,2,4-trimethyl-5-oxoimidazolidin-1-yl)propenamide (8)
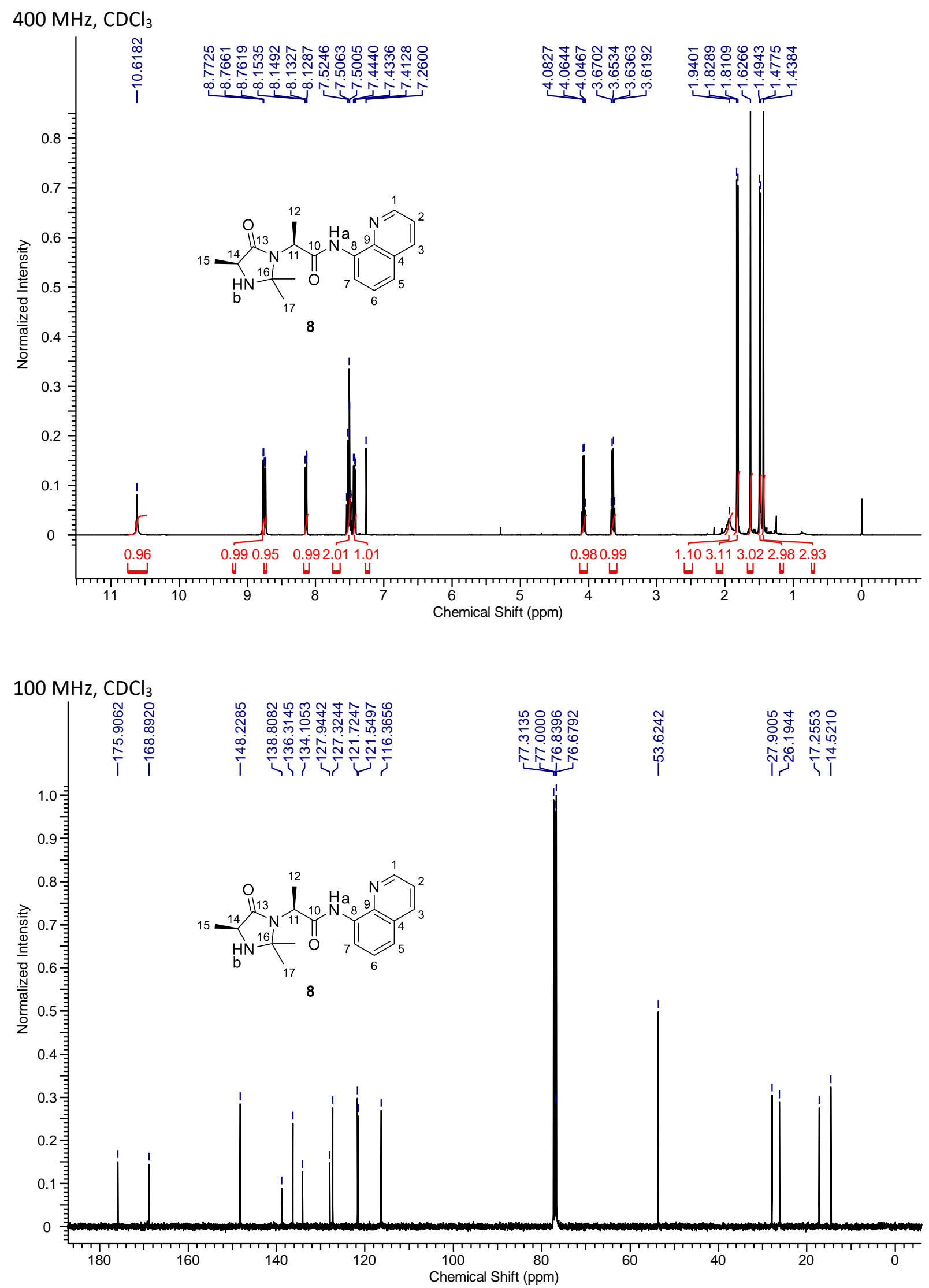
Benzyl (S)-2,2,5-trimethyl-4-oxo-3-((S)-1-oxo-1-(quinolin-8-ylamino)propan-2-yl)imidazolidine-1carboxylate (9)
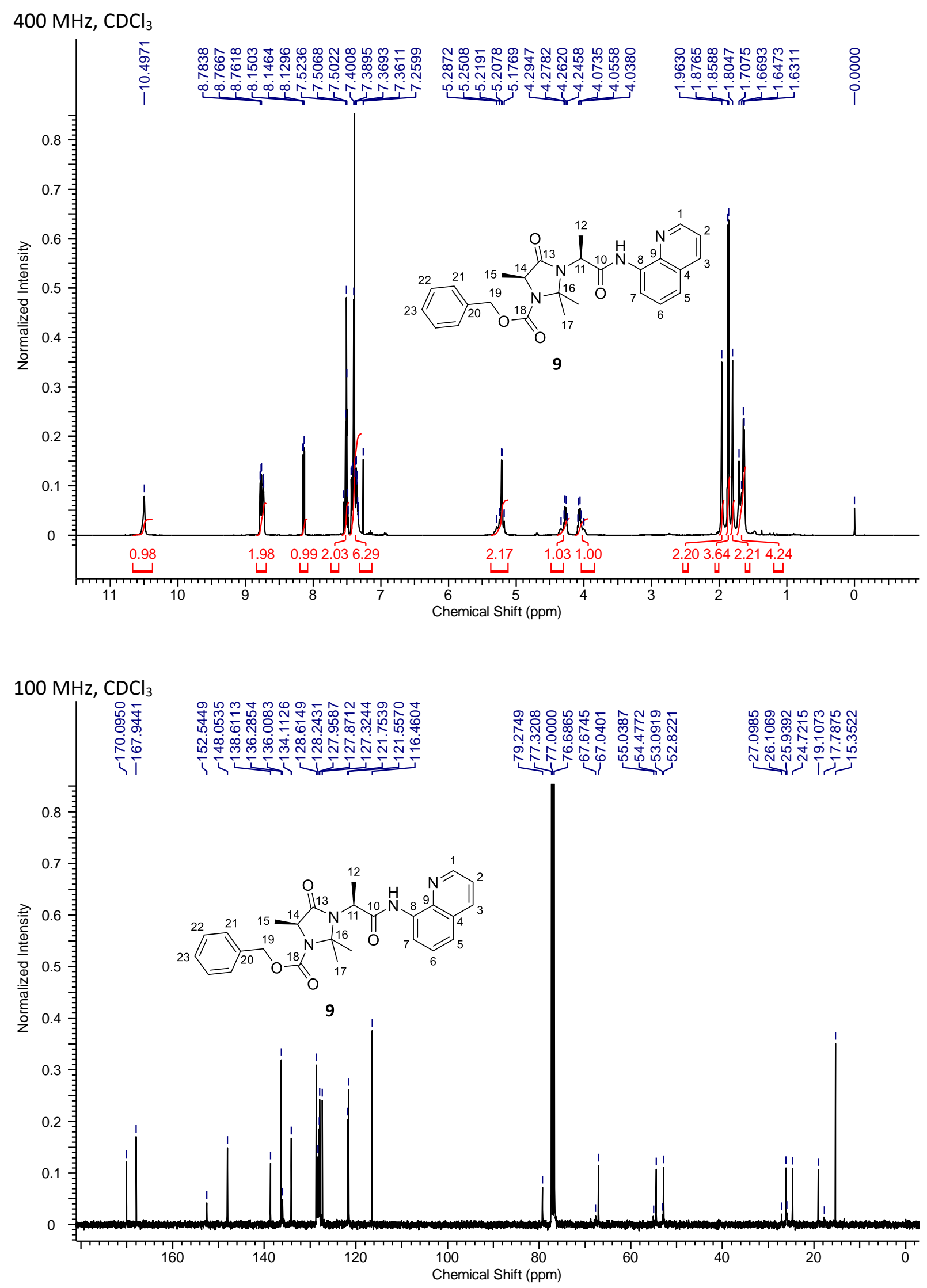
tert-Butyl (S)-(1-((2-(methylthio)phenyl)amino)-1-oxopropan-2-yl)carbamate (11)
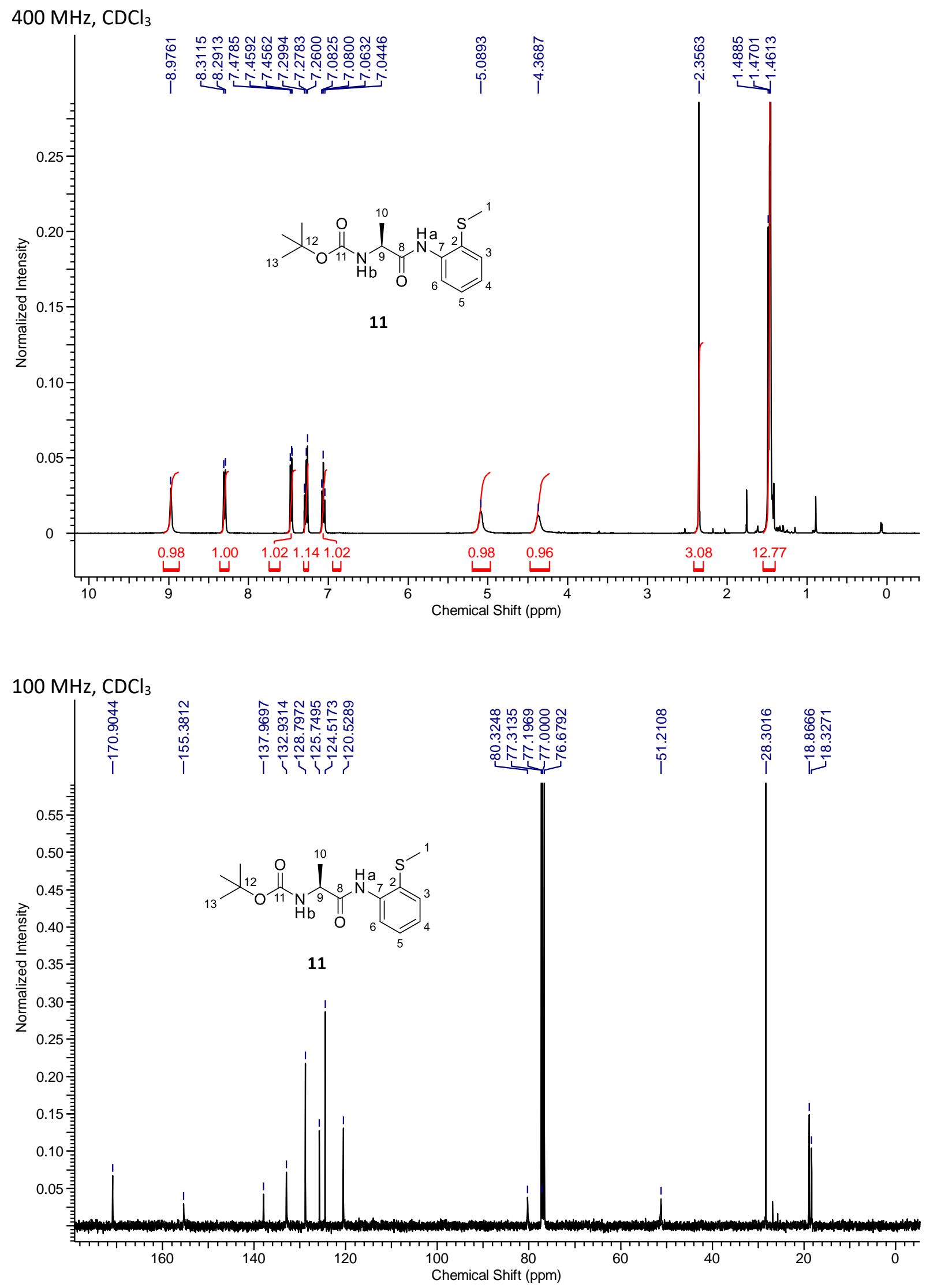
tert-Butyl ((S)-1-(((S)-1-((2-(methylthio)phenyl)amino)-1-oxopropan-2-yl)amino)-1-oxopropan-2-yl)carbamate (12)
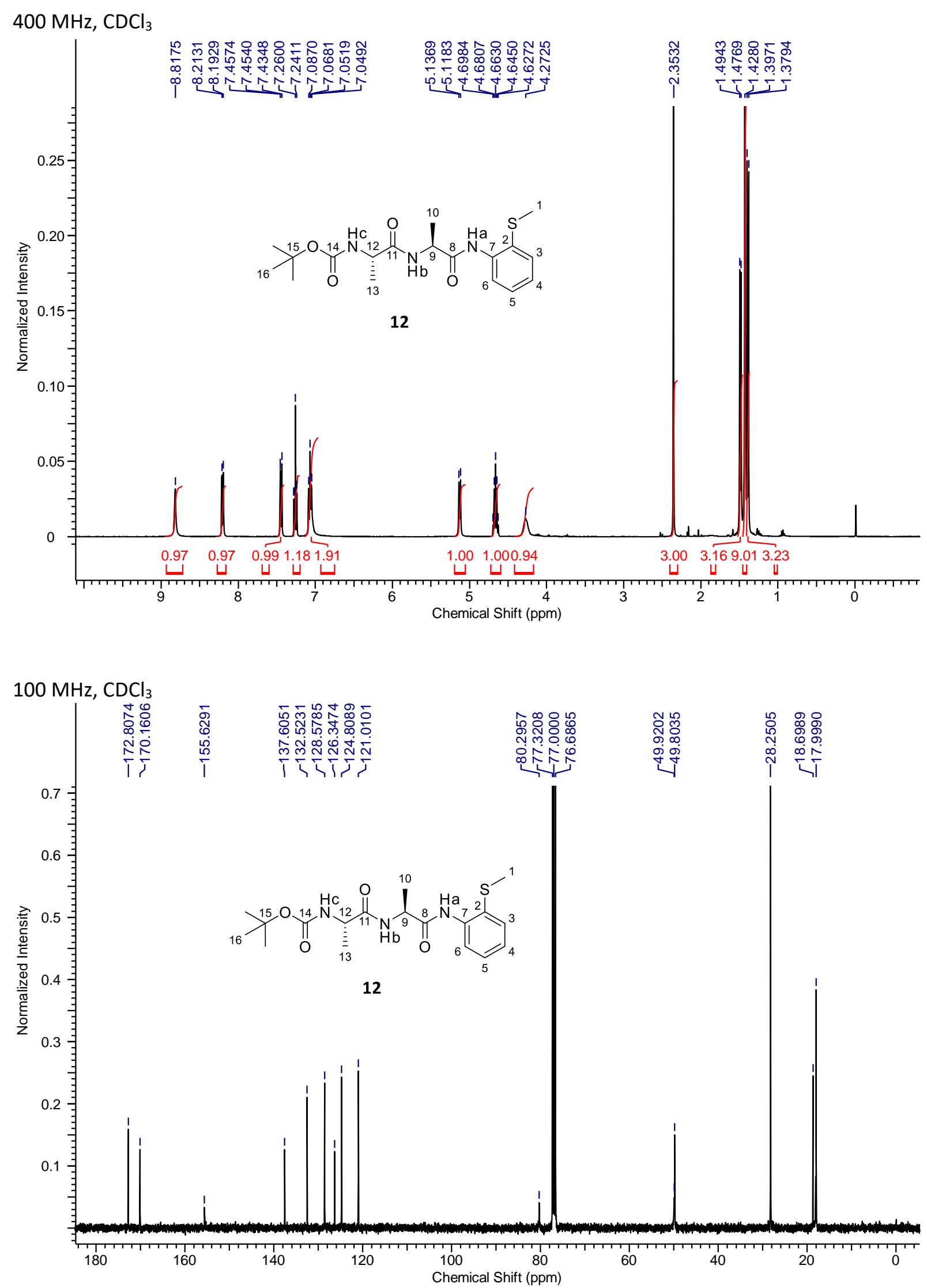
Benzyl (S)-2,2,5-trimethyl-3-((S)-1-((2-(methylthio)phenyl)amino)-1-oxopropan-2-yl)-4-oxoimidazolidine-1-carboxylate (13)

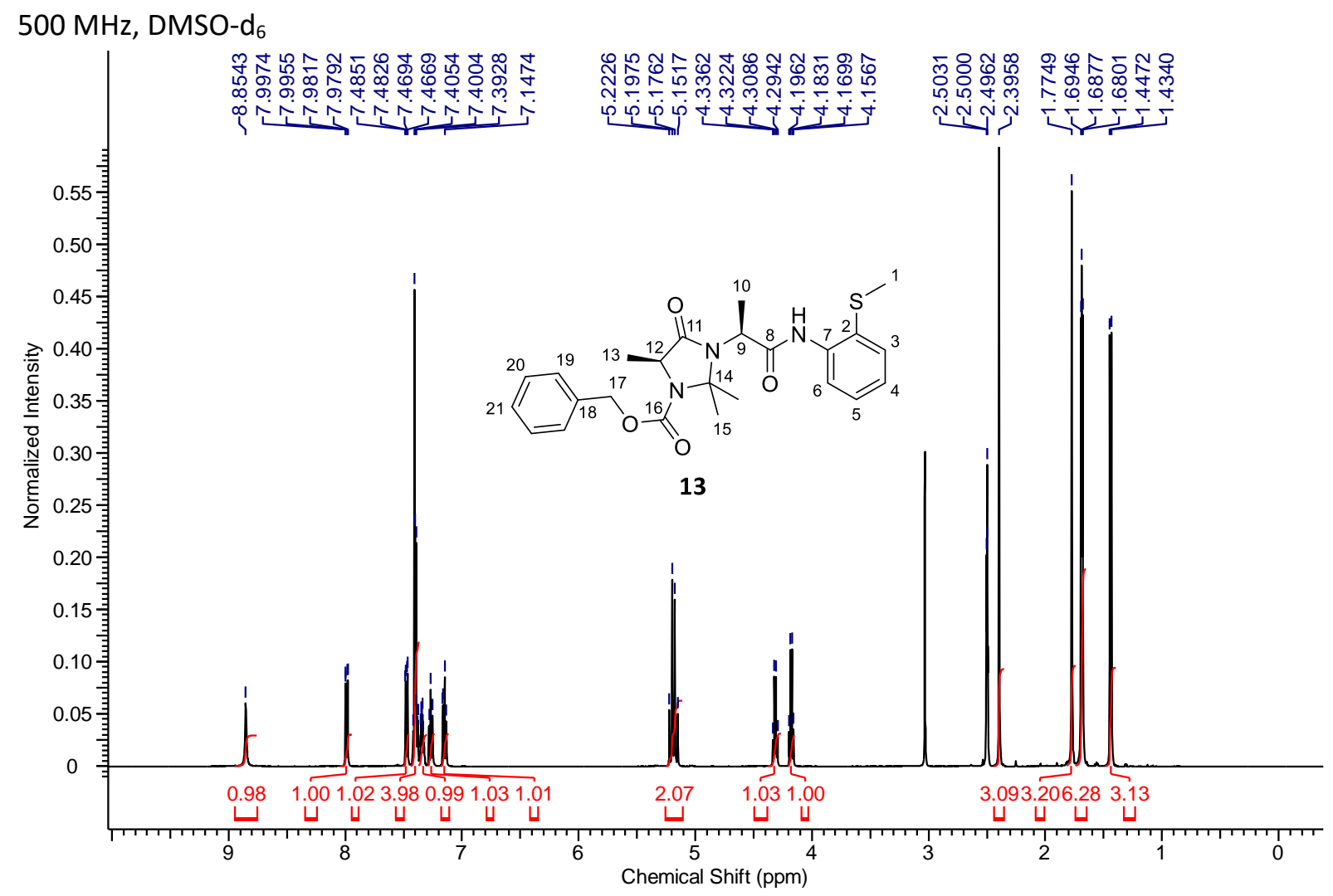

$125 \mathrm{MHz}, \mathrm{DMSO}-\mathrm{d}_{6}$

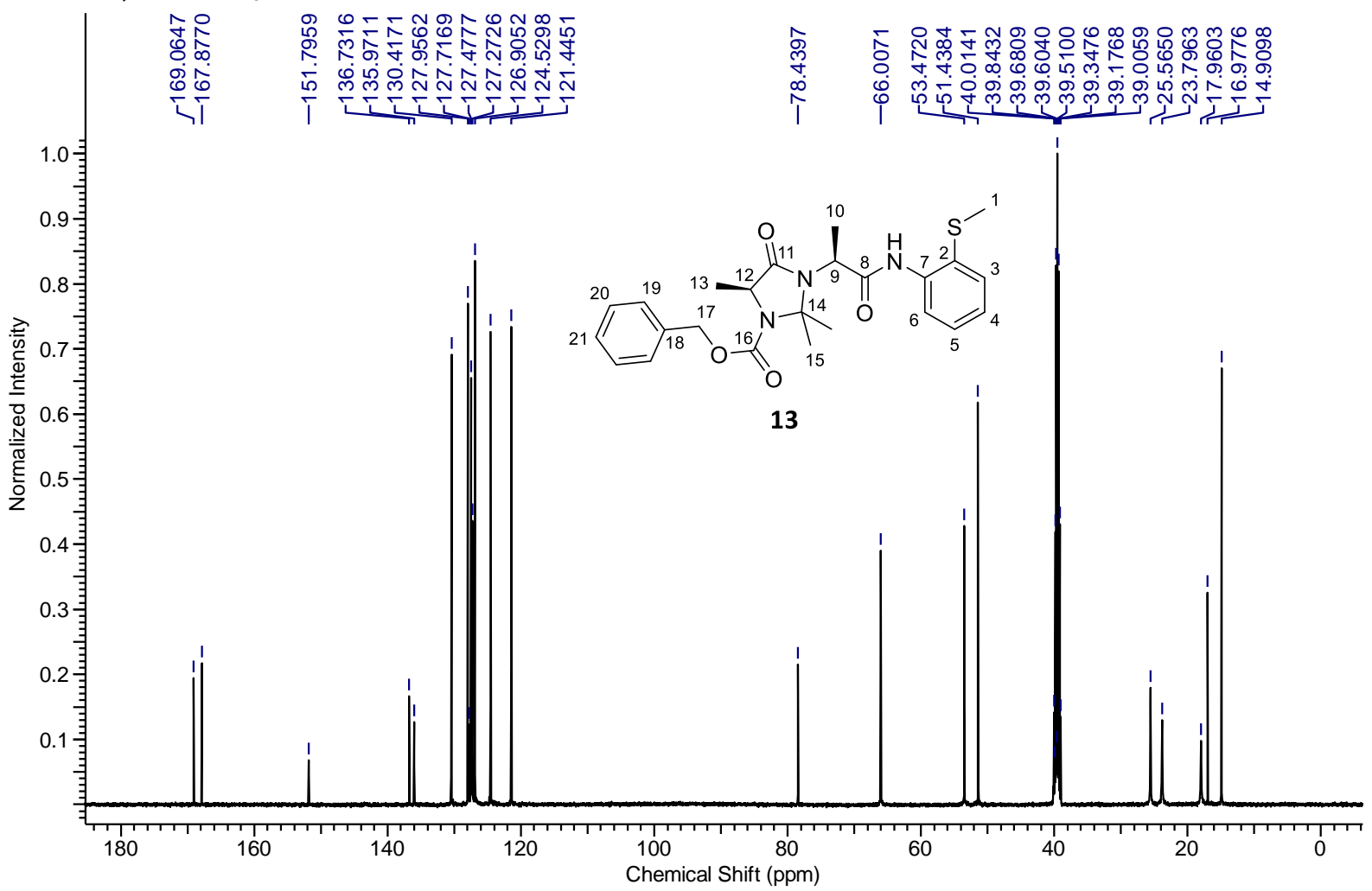


(S)-2-((S)-3-((Benzyloxy)carbonyl)-2,2,4-trimethyl-5-oxoimidazolidin-1-yl)propanoic acid (14)
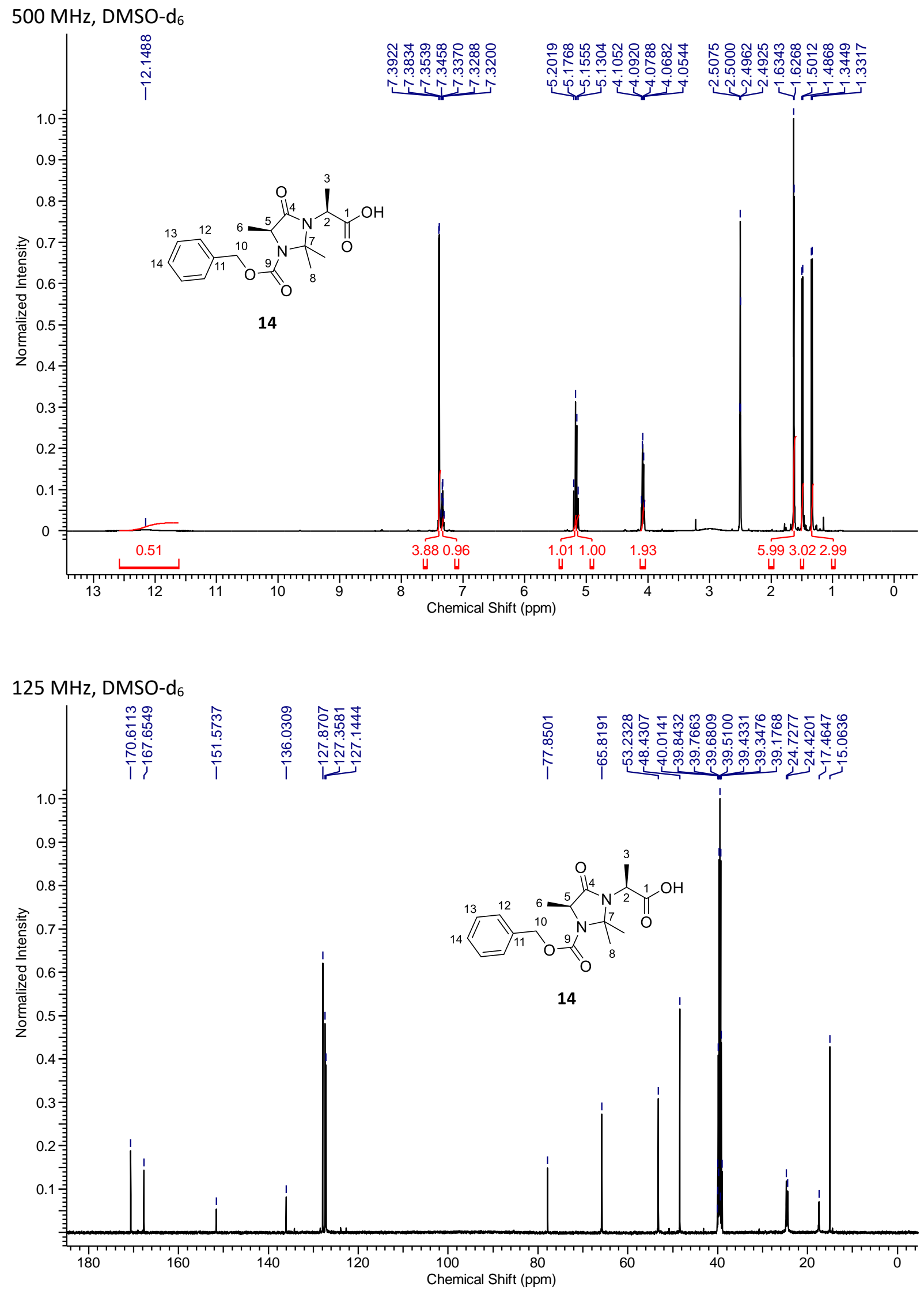
Benzyl (S)-2,2,5-trimethyl-3-((S)-1-((2-(methylthio)phenyl)amino)-1-oxo-3-(p-tolyl)propan-2-yl)-4oxoimidazolidine-1-carboxylate (15a)

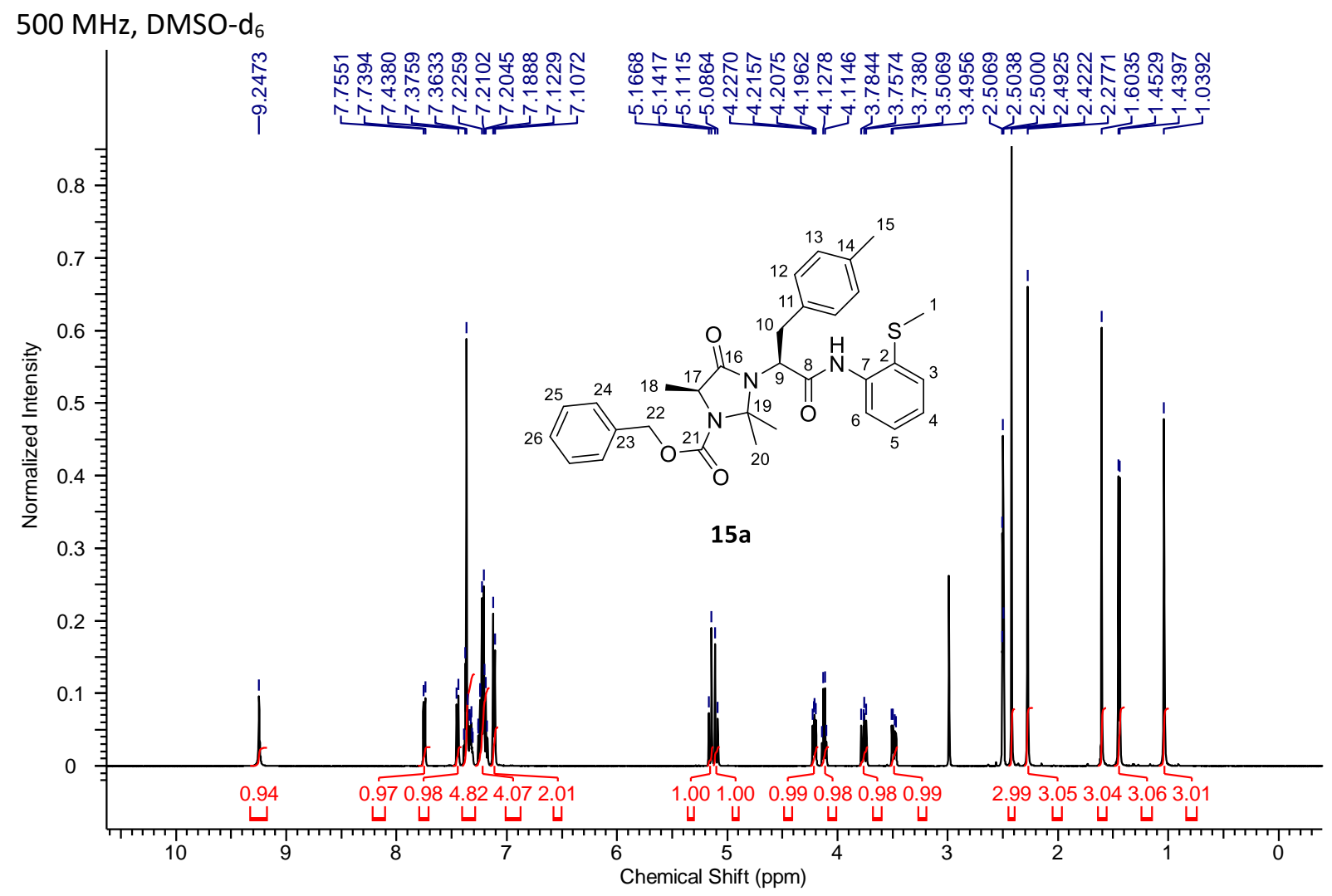

$125 \mathrm{MHz}, \mathrm{DMSO}-\mathrm{d}_{6}$

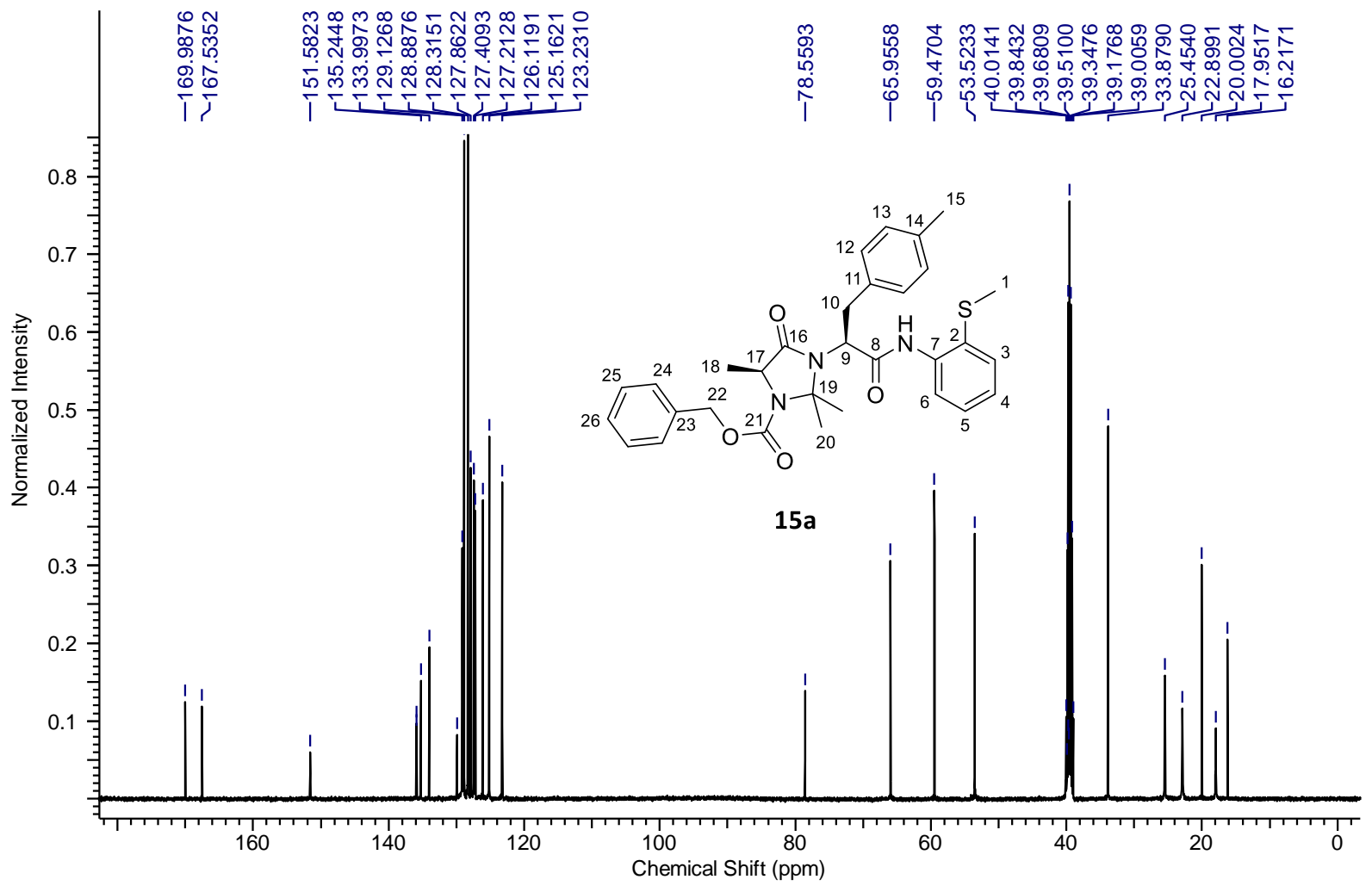


Crude product 15 a (no second diastereomer, only traces of starting material 13)

$500 \mathrm{MHz}, \mathrm{DMSO}-\mathrm{d}_{6}$

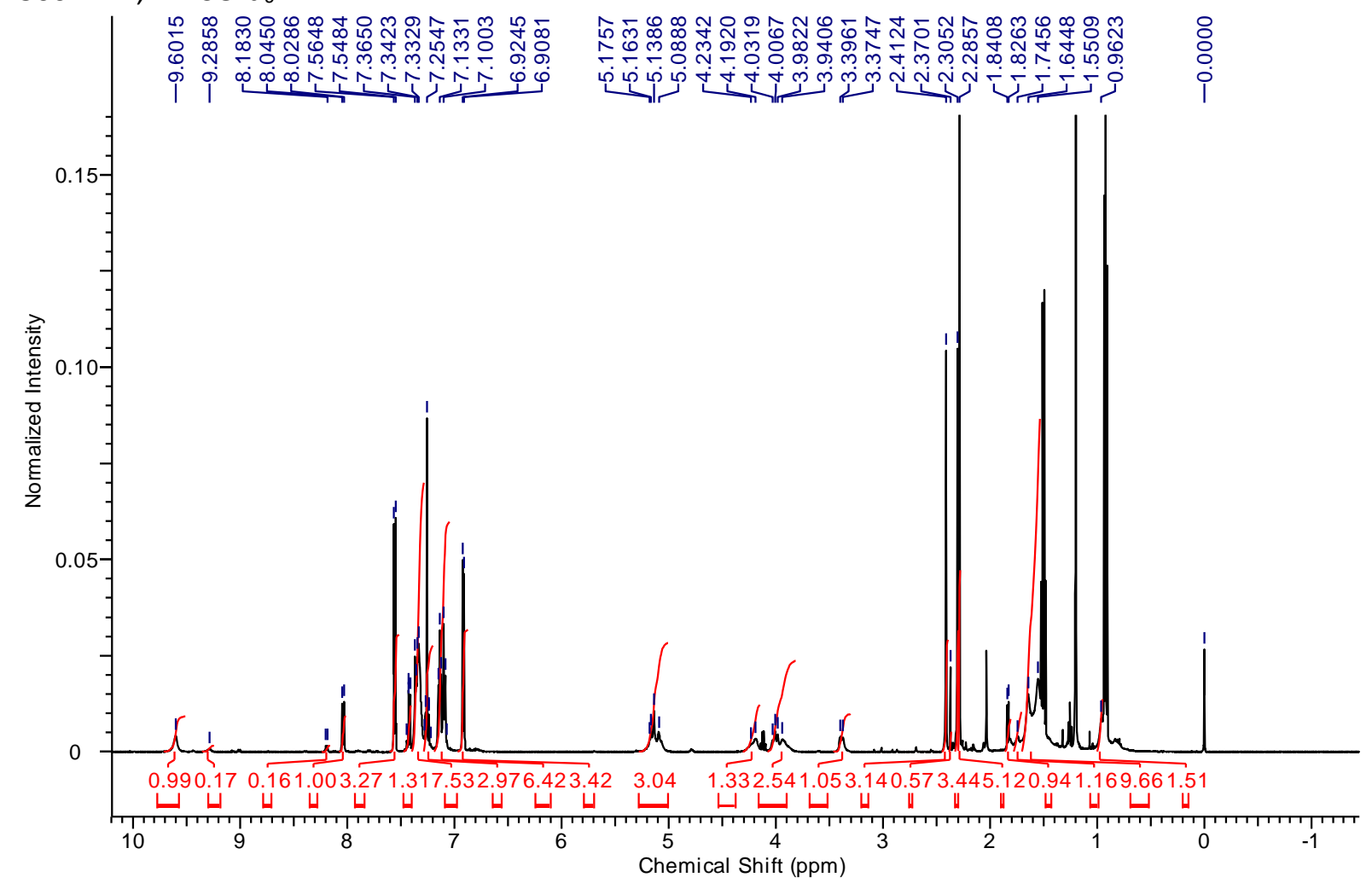

$500 \mathrm{MHz}, \mathrm{DMSO}-\mathrm{d}_{6}$

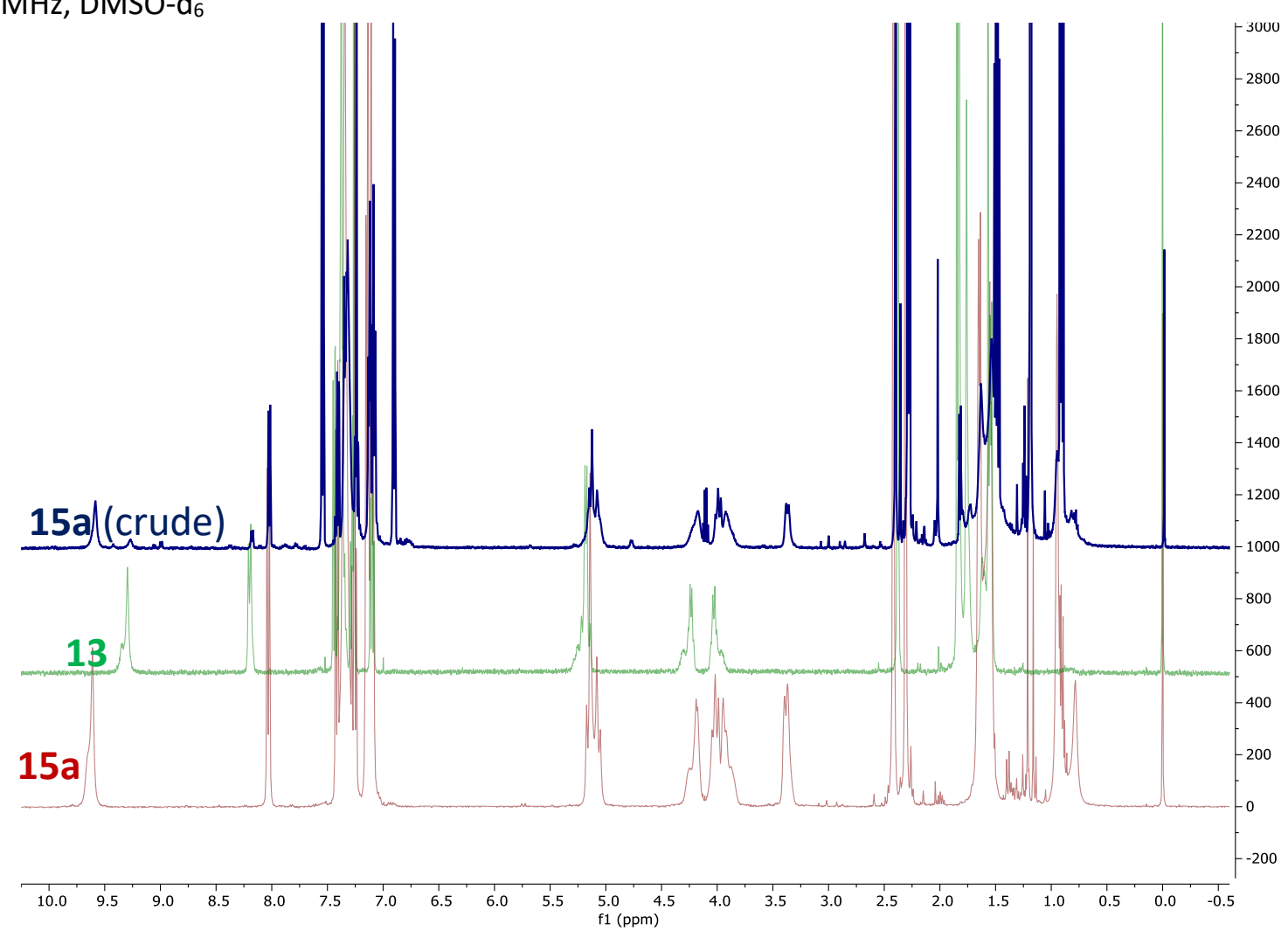


Benzyl (S)-2,2,5-trimethyl-3-((S)-1-((2-(methylthio)phenyl)amino)-1-oxo-3-phenylpropan-2-yl)-4oxoimidazolidine-1-carboxylate (15b)

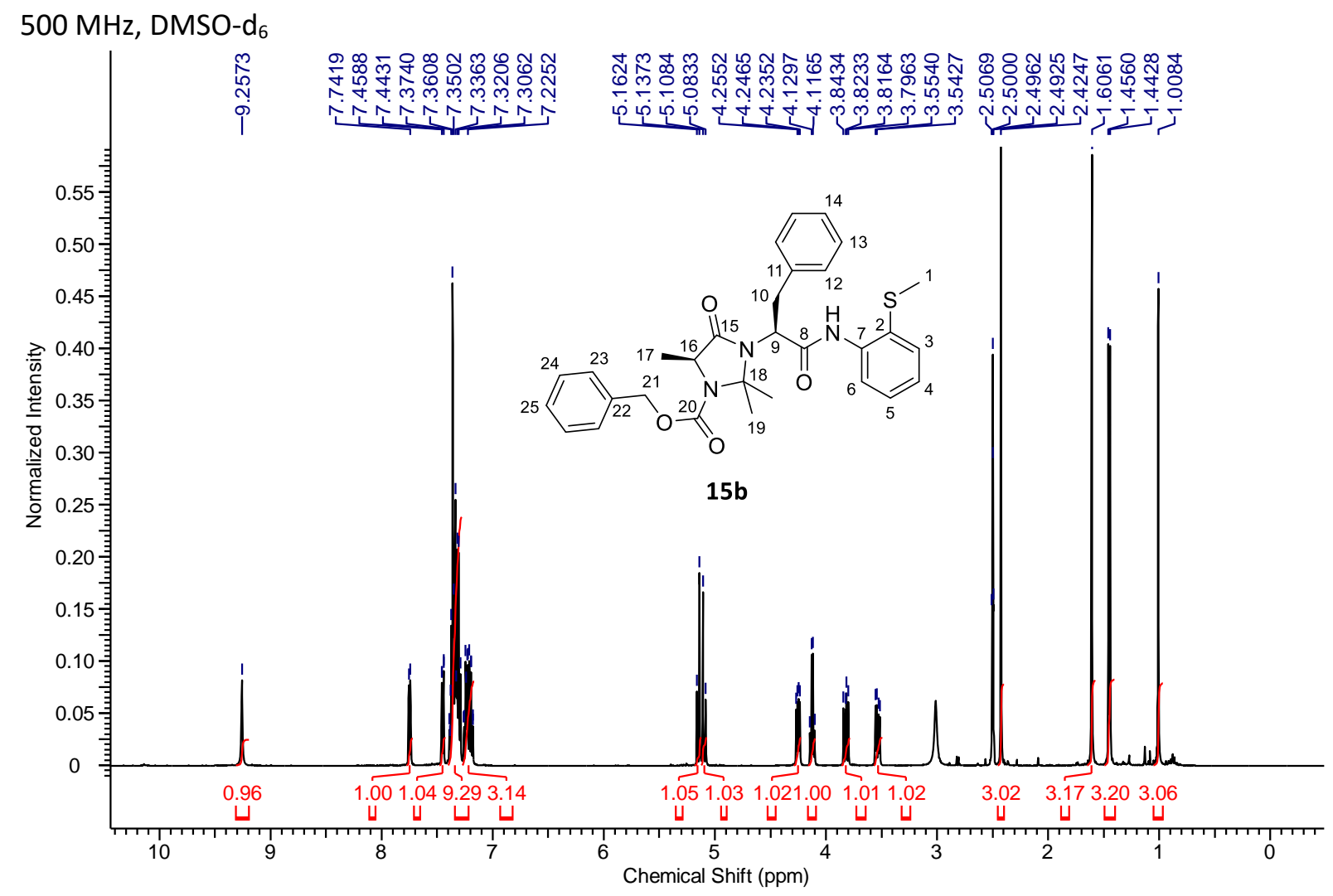

$125 \mathrm{MHz}, \mathrm{DMSO}-\mathrm{d}_{6}$

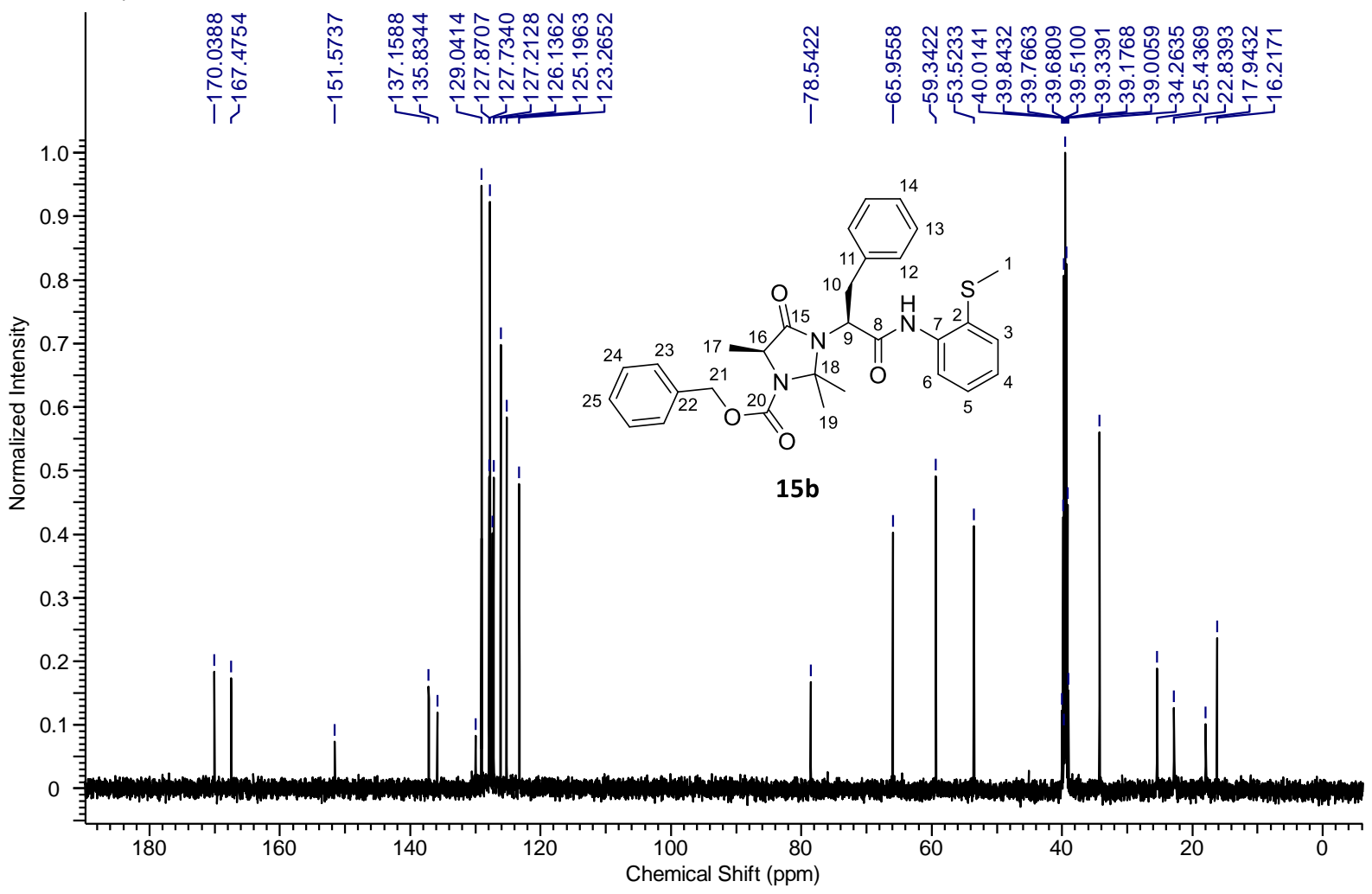


Benzyl (S)-3-((S)-3-(4-methoxyphenyl)-1-((2-(methylthio)phenyl)amino)-1-oxopropan-2-yl)-2,2,5-trimethyl-4-oxoimidazolidine-1-carboxylate (15c)
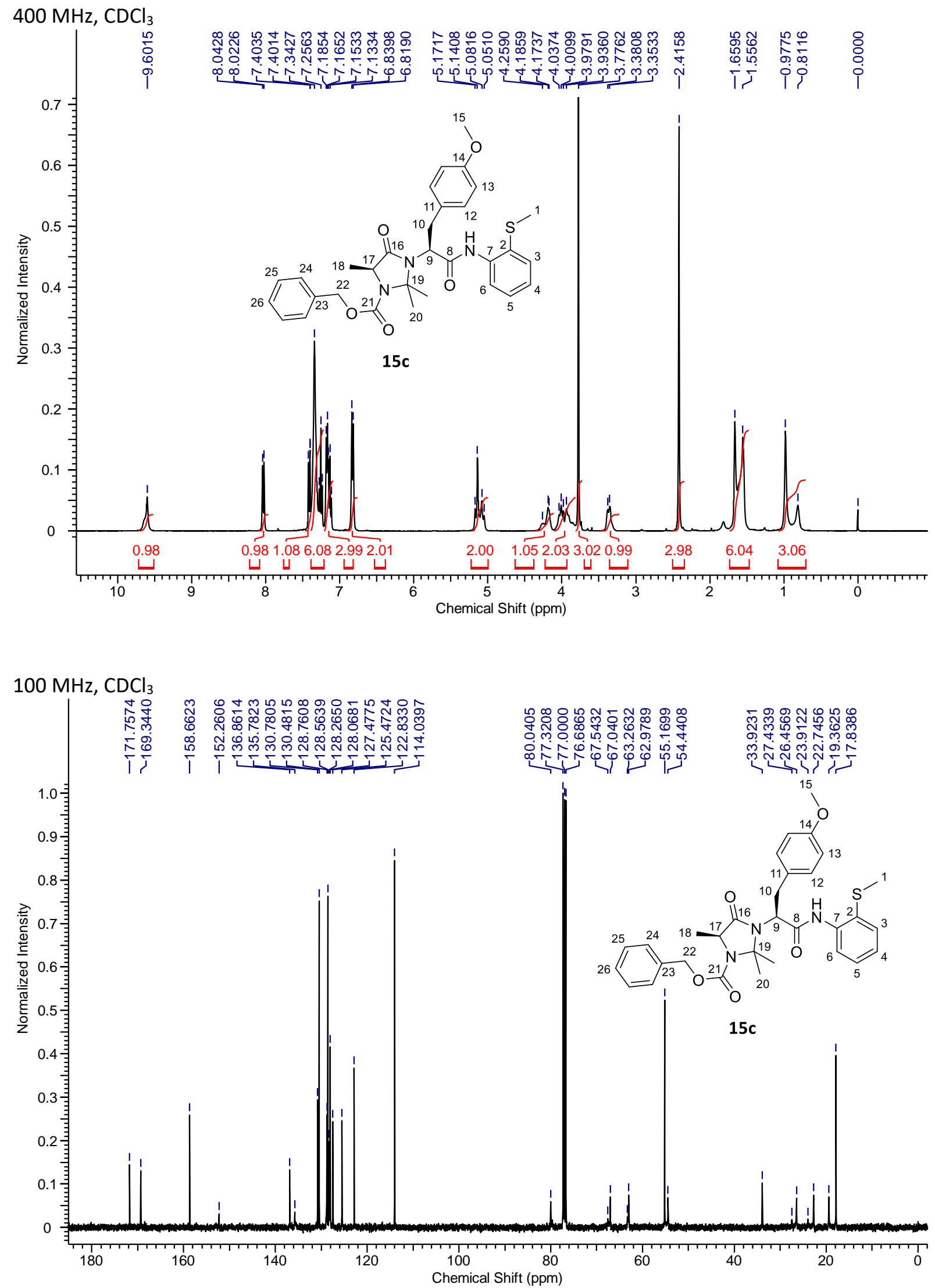
Benzyl (S)-3-((S)-3-(4-chlorophenyl)-1-((2-(methylthio)phenyl)amino)-1-oxopropan-2-yl)-2,2,5-trimethyl-4-oxoimidazolidine-1-carboxylate (15d)

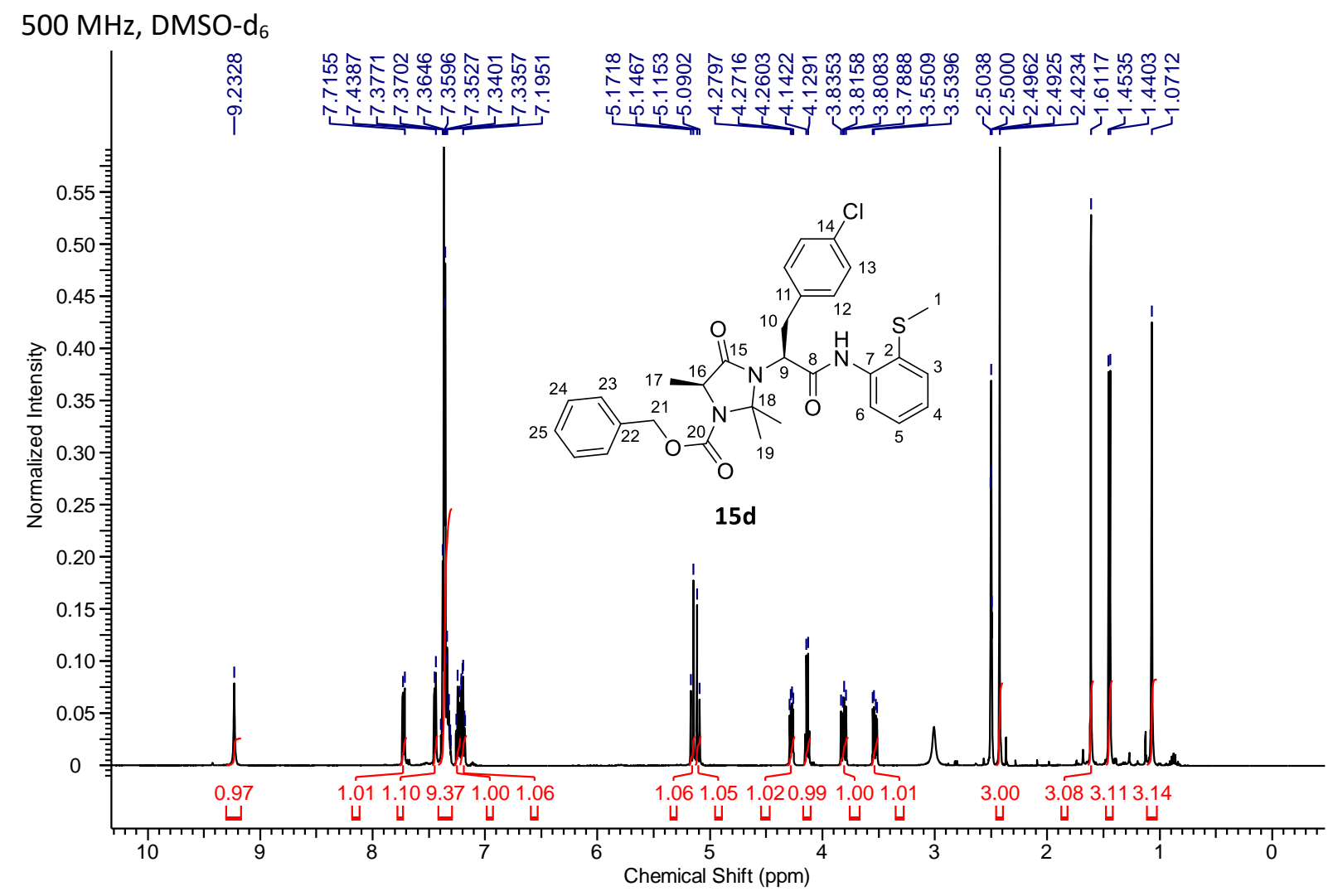

$125 \mathrm{MHz}, \mathrm{DMSO}-\mathrm{d}_{6}$

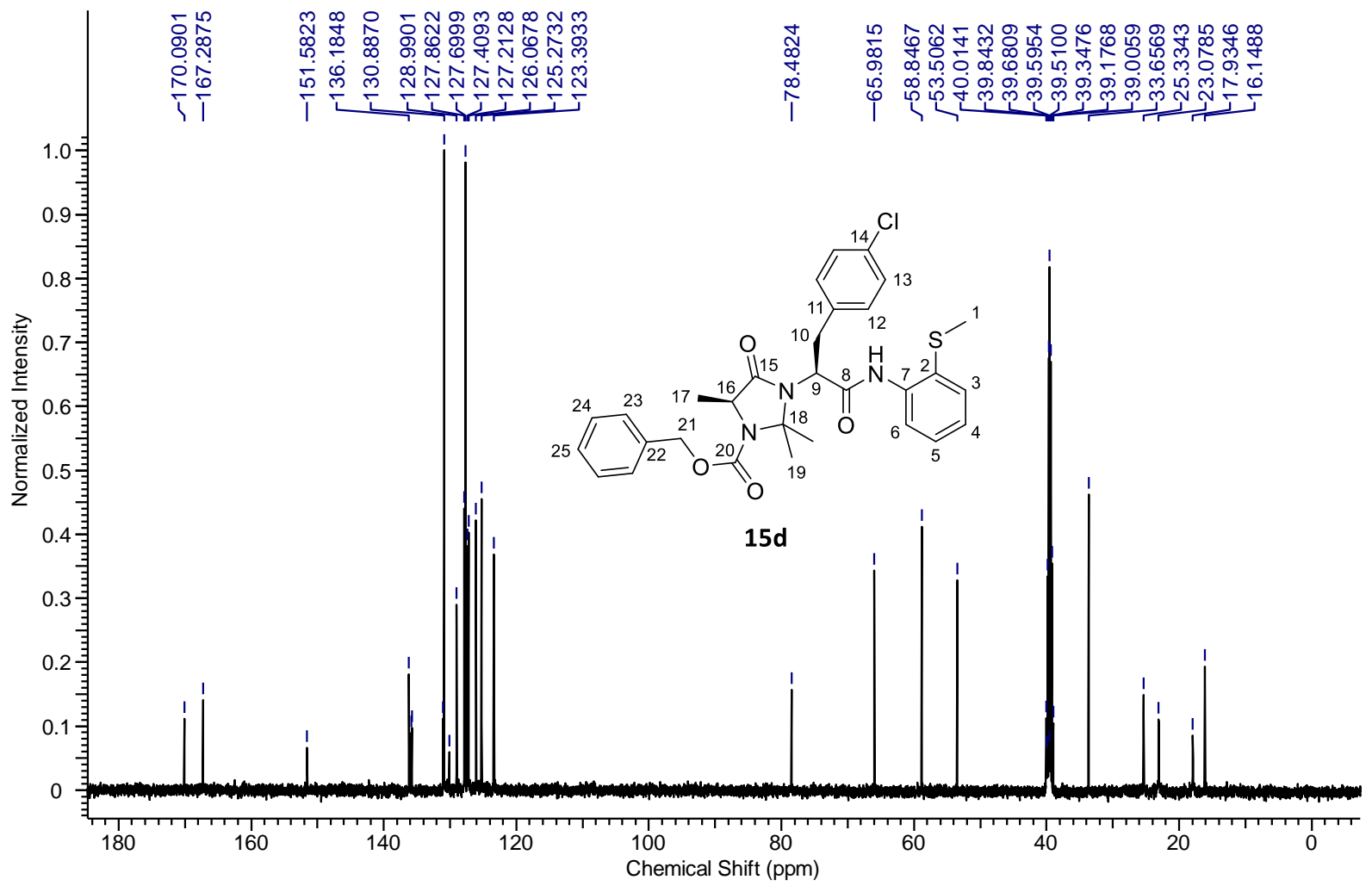


Benzyl (S)-3-((S)-3-(4-bromophenyl)-1-((2-(methylthio)phenyl)amino)-1-oxopropan-2-yl)-2,2,5-trimethyl-4-oxoimidazolidine-1-carboxylate (15e)

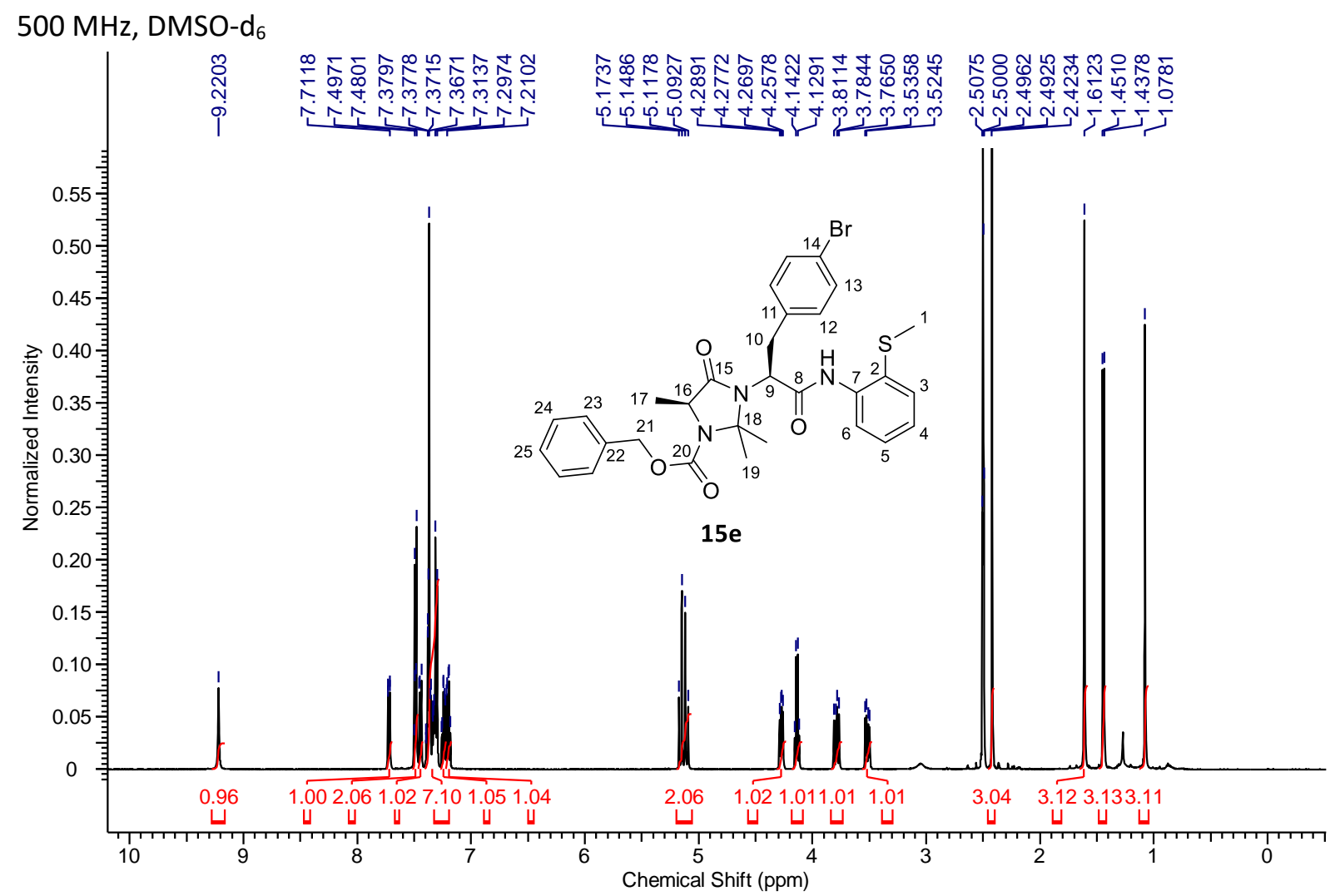

$125 \mathrm{MHz}, \mathrm{DMSO}-\mathrm{d}_{6}$

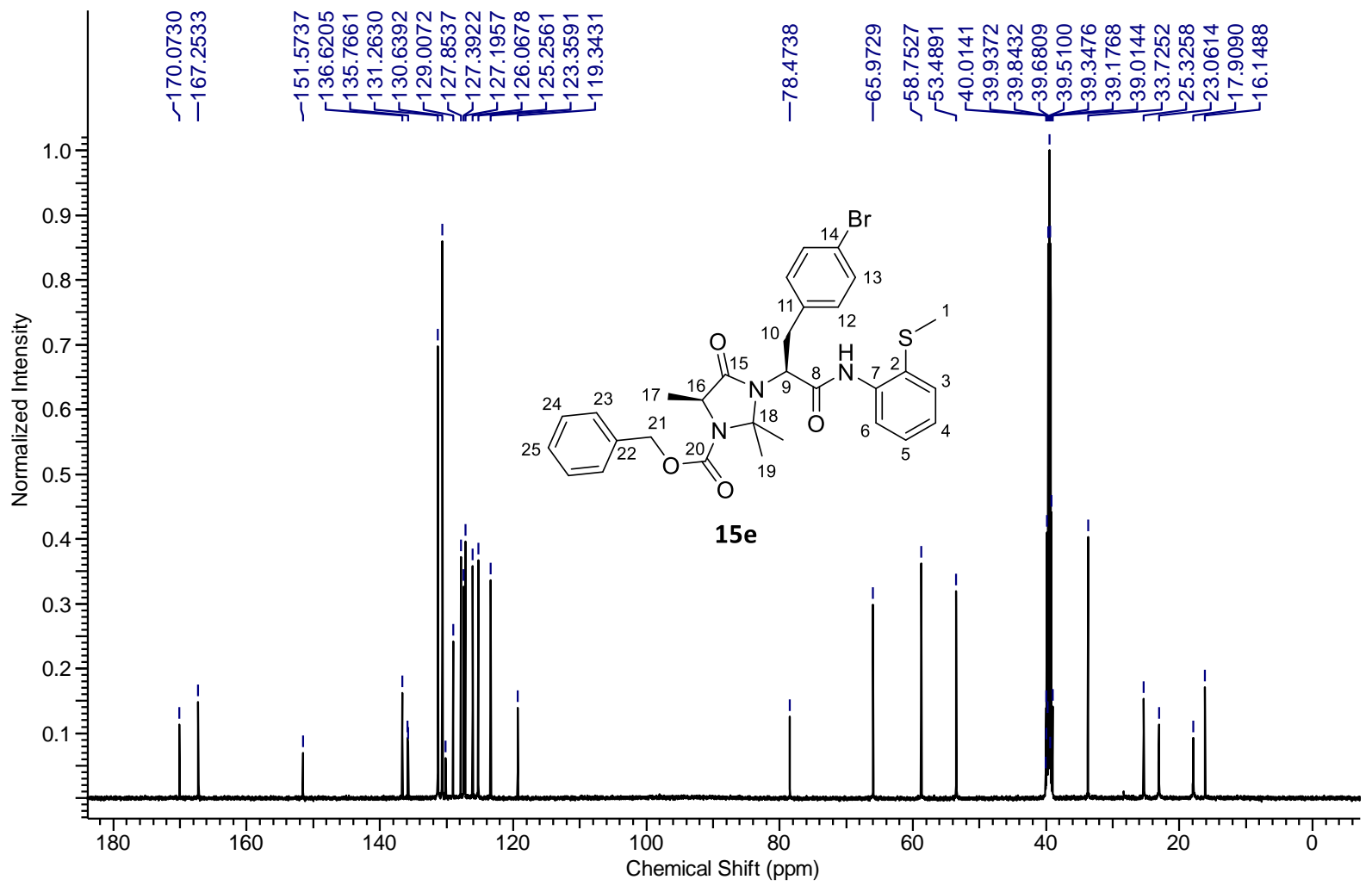


Benzyl (S)-3-((S)-3-(4-iodophenyl)-1-((2-(methylthio)phenyl)amino)-1-oxopropan-2-yl)-2,2,5-trimethyl-4-oxoimidazolidine-1-carboxylate (15f)
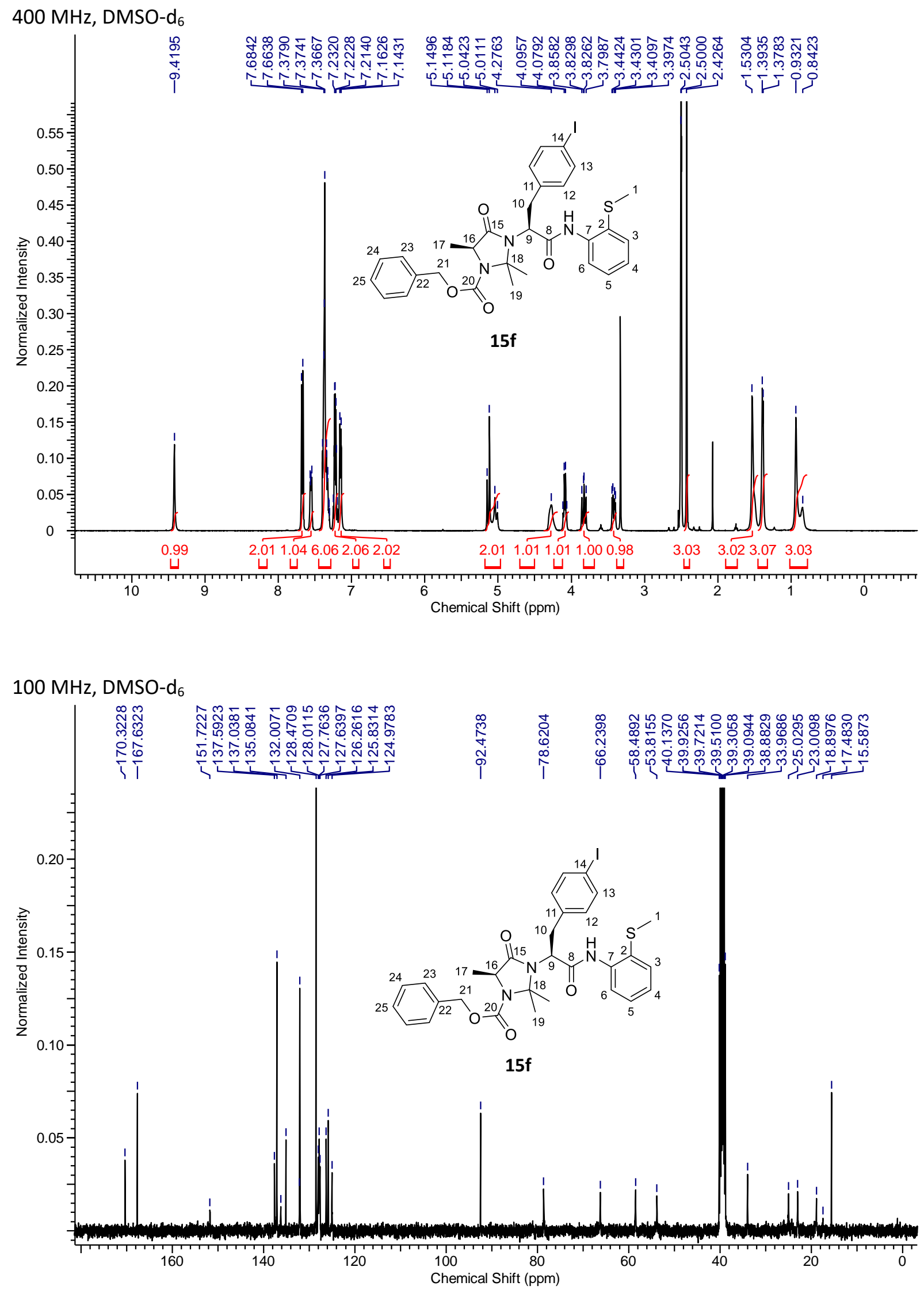
Benzyl (S)-3-((S)-3-(2-fluorophenyl)-1-((2-(methylthio)phenyl)amino)-1-oxopropan-2-yl)-2,2,5-trimethyl-4-oxoimidazolidine-1-carboxylate $(15 \mathrm{~g})$

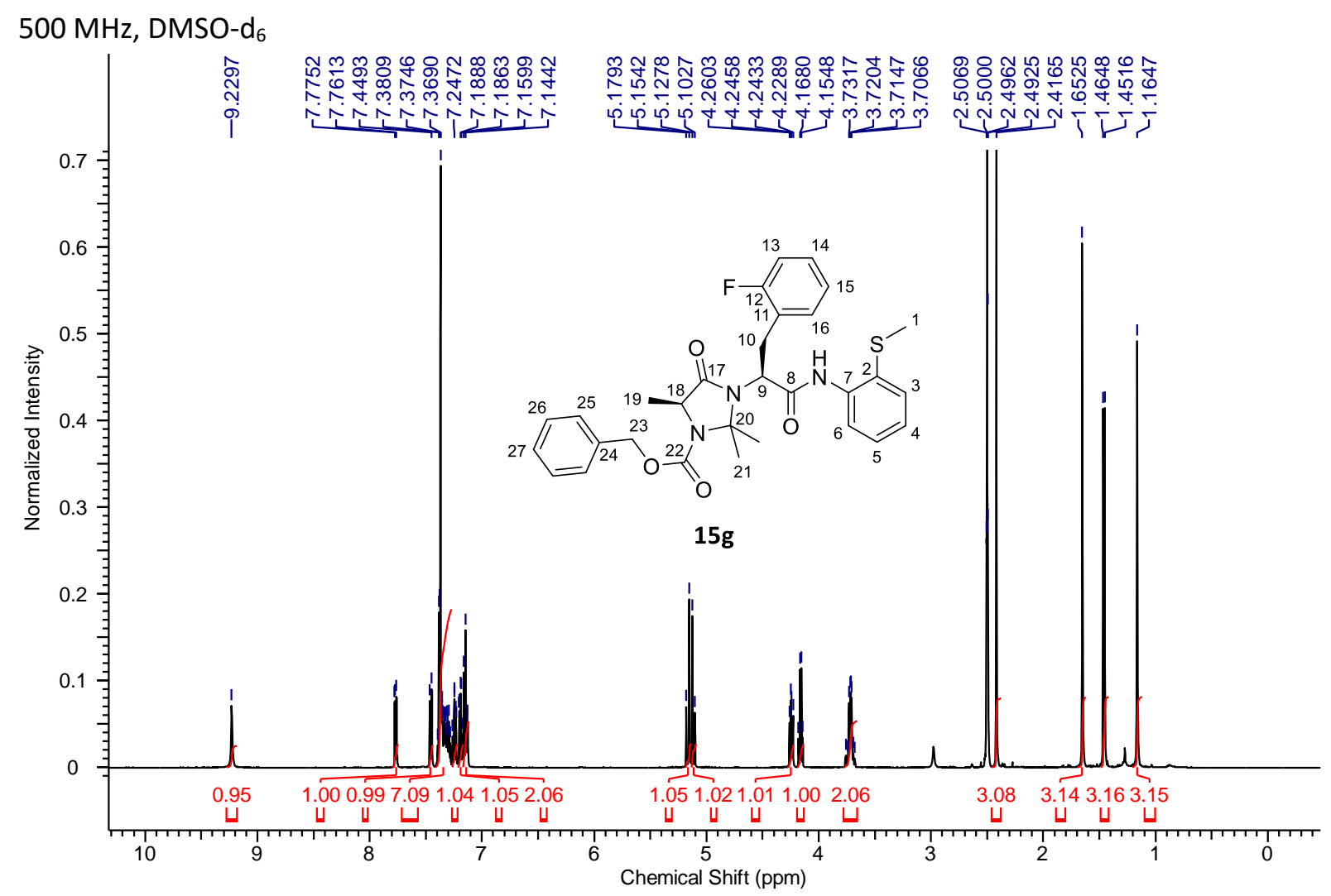

$125 \mathrm{MHz}, \mathrm{DMSO}-\mathrm{d}_{6}$

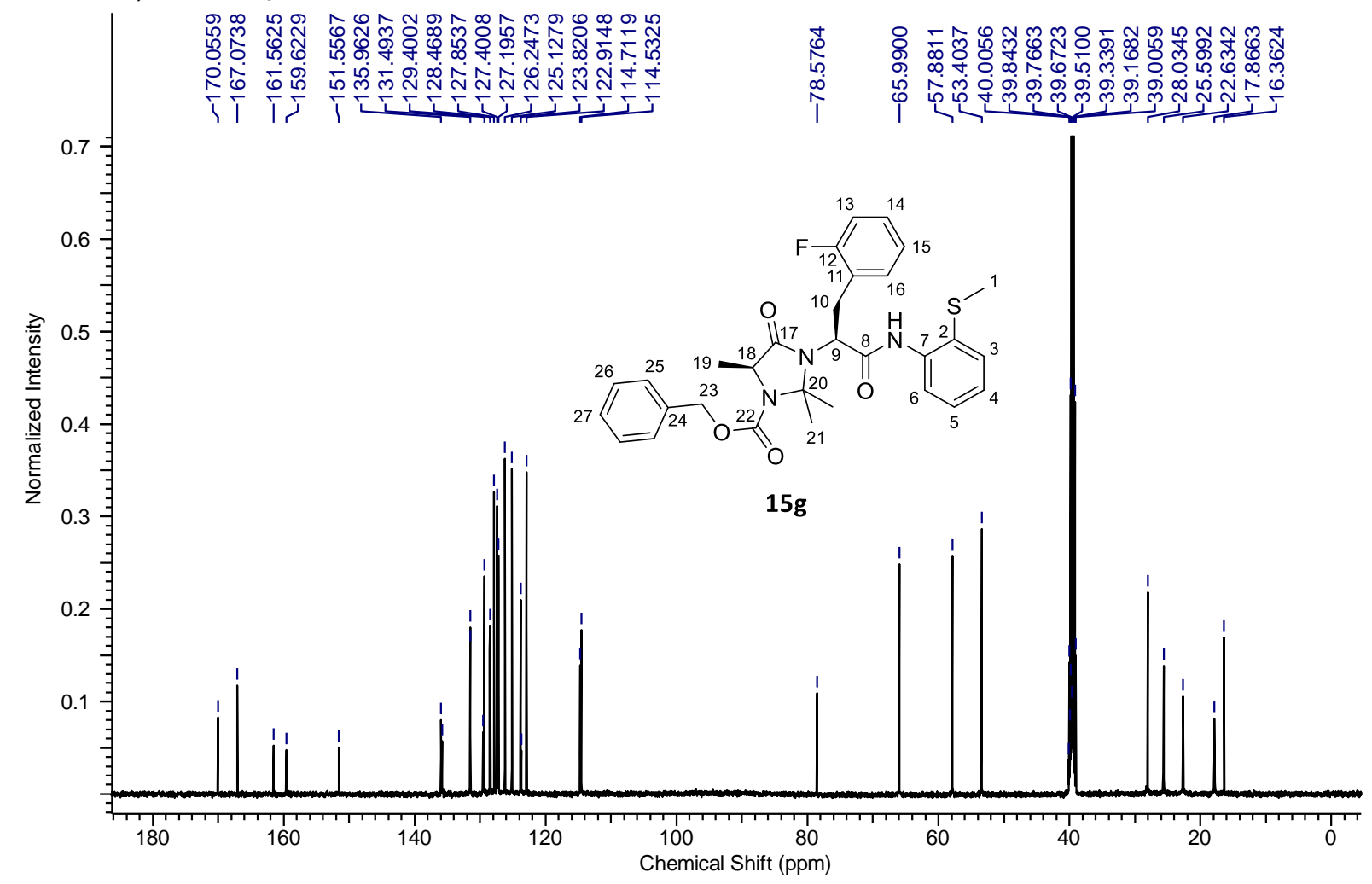


Benzyl (S)-3-((S)-3-(4-formylphenyl)-1-((2-(methylthio)phenyl)amino)-1-oxopropan-2-yl)-2,2,5-trimethyl-4-oxoimidazolidine-1-carboxylate (15h)

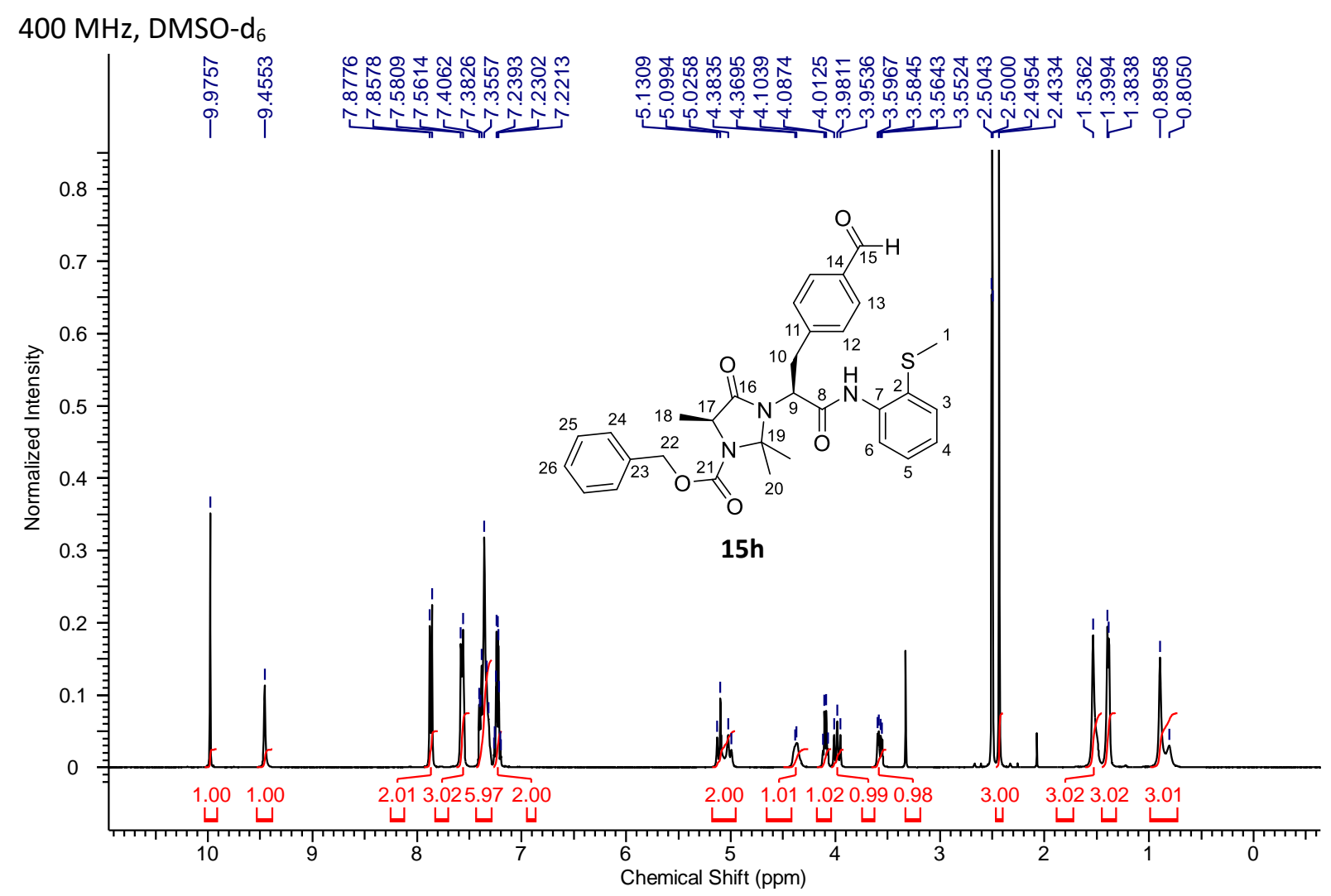

$100 \mathrm{MHz}, \mathrm{DMSO}-\mathrm{d}_{6}$

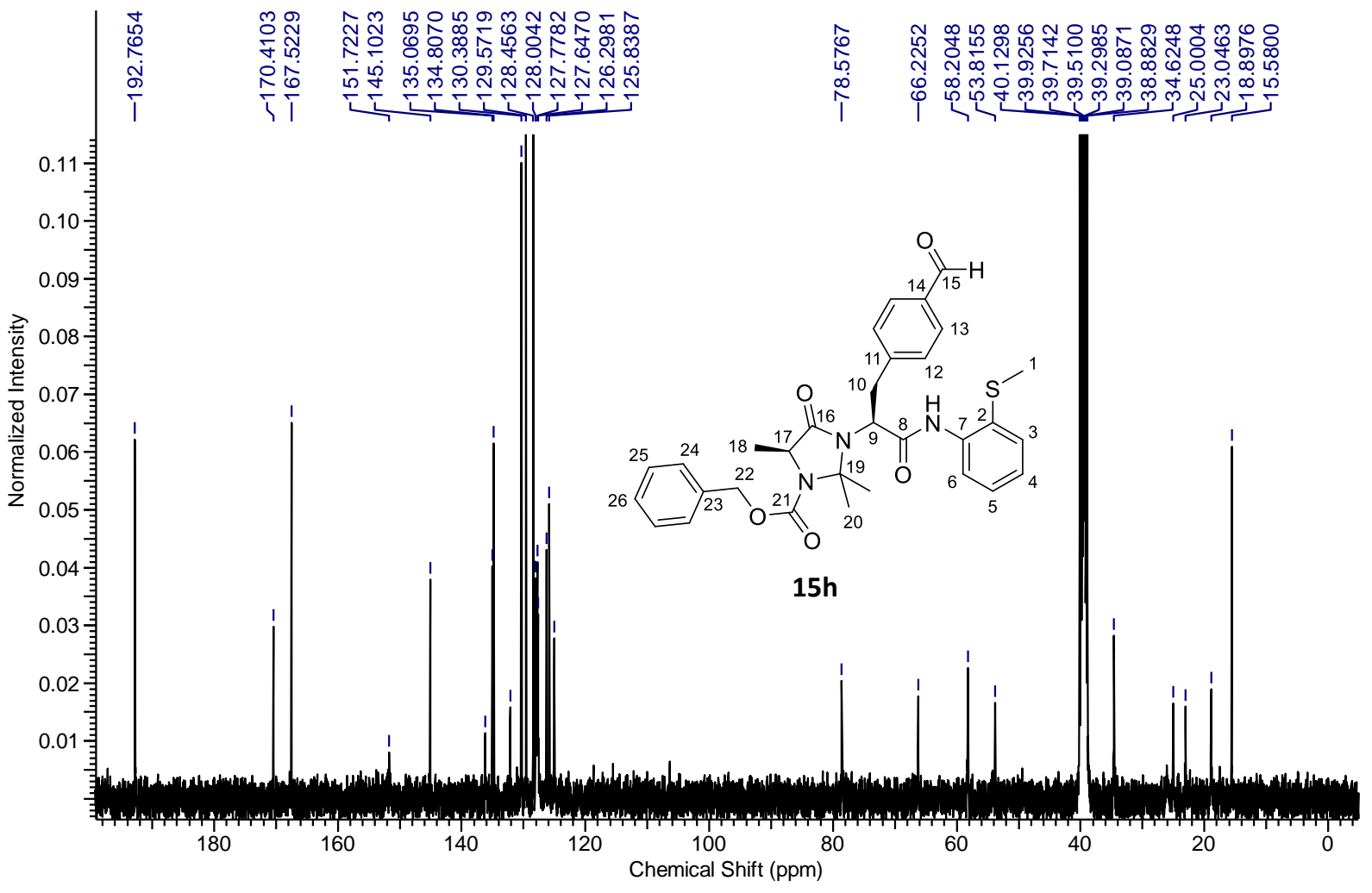


Benzyl (S)-2,2,5-trimethyl-3-((S)-1-((2-(methylthio)phenyl)amino)-3-(4-nitrophenyl)-1-oxopropan-2yl)-4-oxoimidazolidine-1-carboxylate (15i)

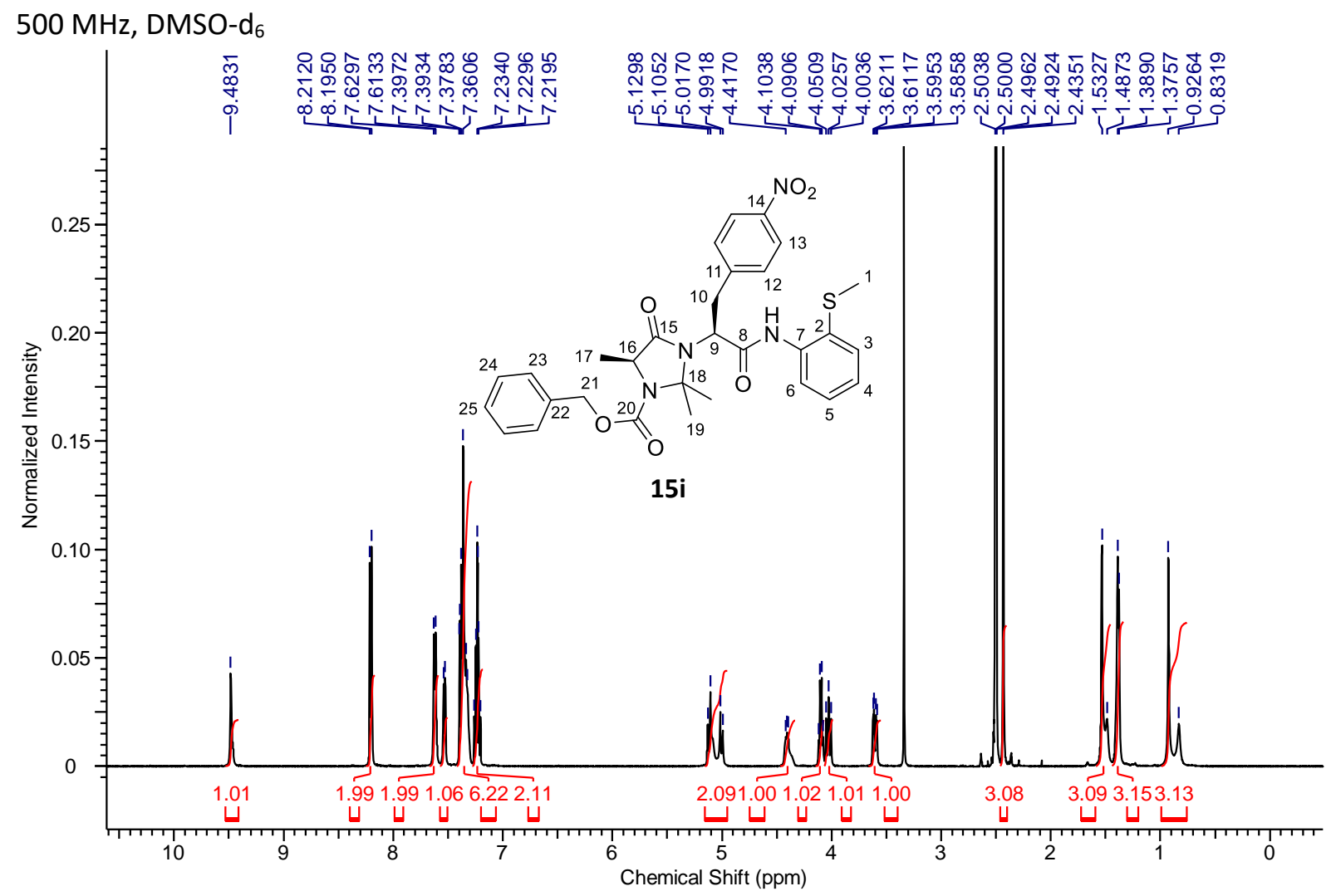

$125 \mathrm{MHz}, \mathrm{DMSO}-\mathrm{d}_{6}$

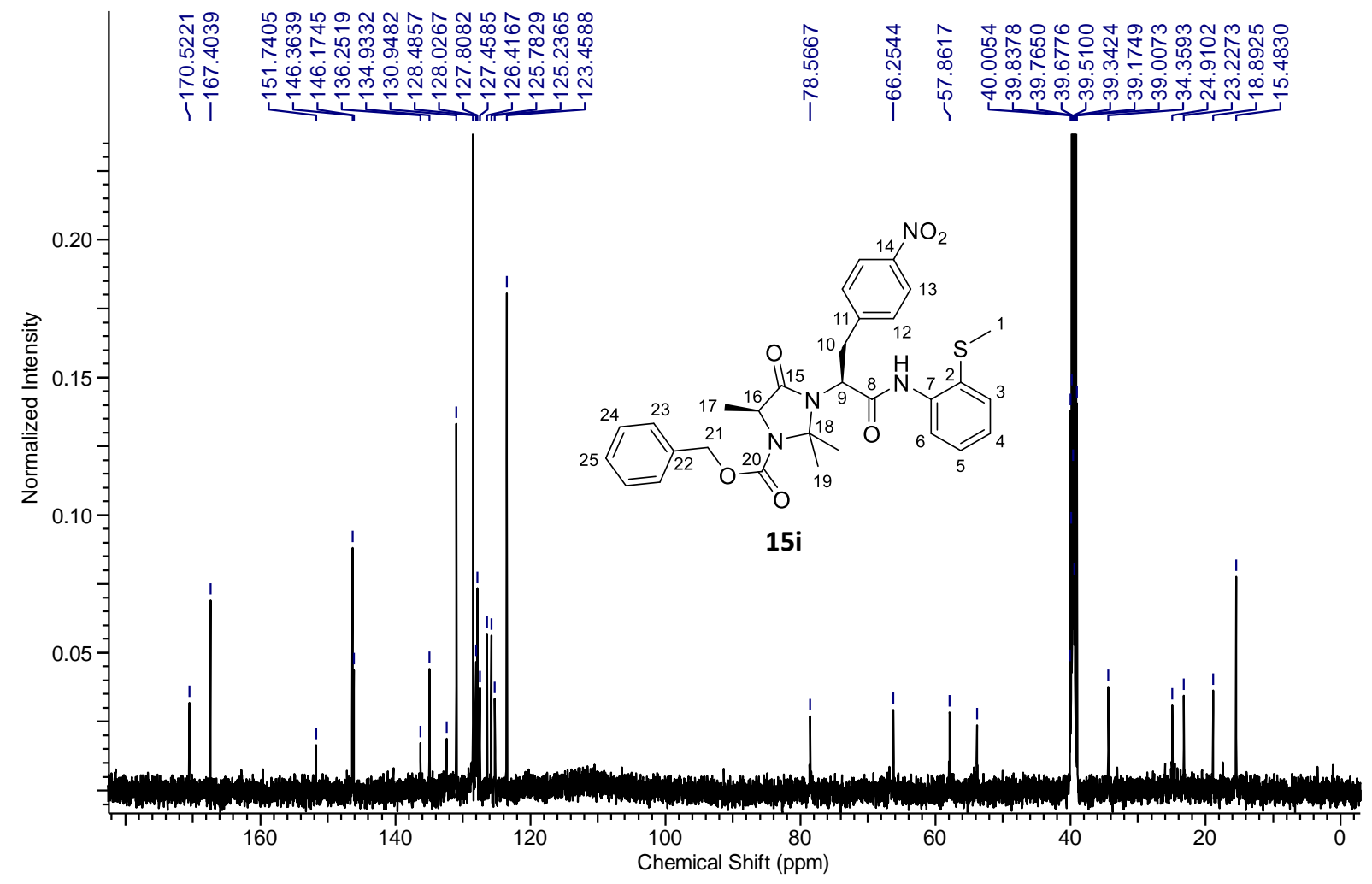


Benzyl (S)-3-((S)-3-(1H-indol-5-yl)-1-((2-(methylthio)phenyl)amino)-1-oxopropan-2-yl)-2,2,5-trimethyl-4-oxoimidazolidine-1-carboxylate (15k)

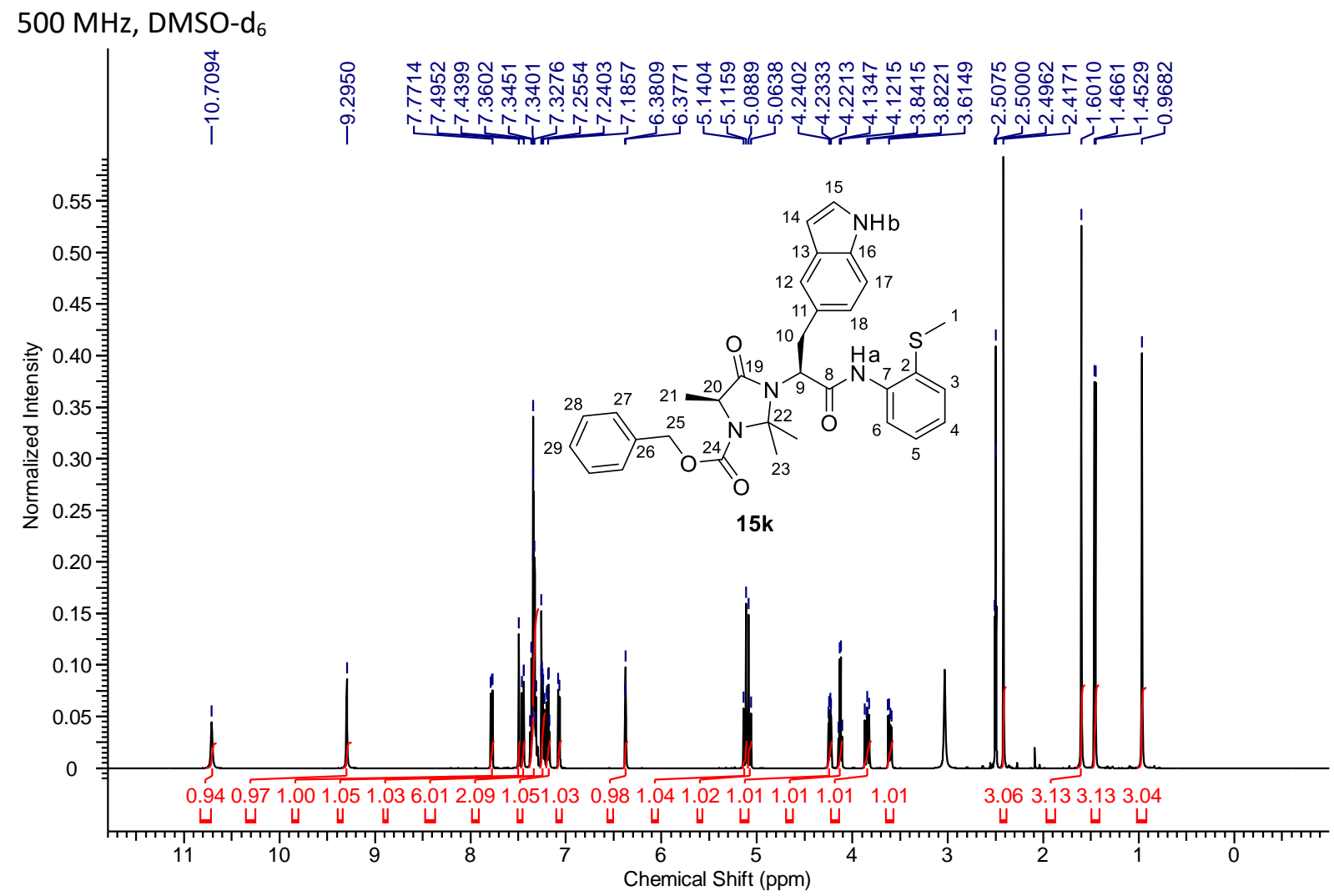

$125 \mathrm{MHz}, \mathrm{DMSO}-\mathrm{d}_{6}$

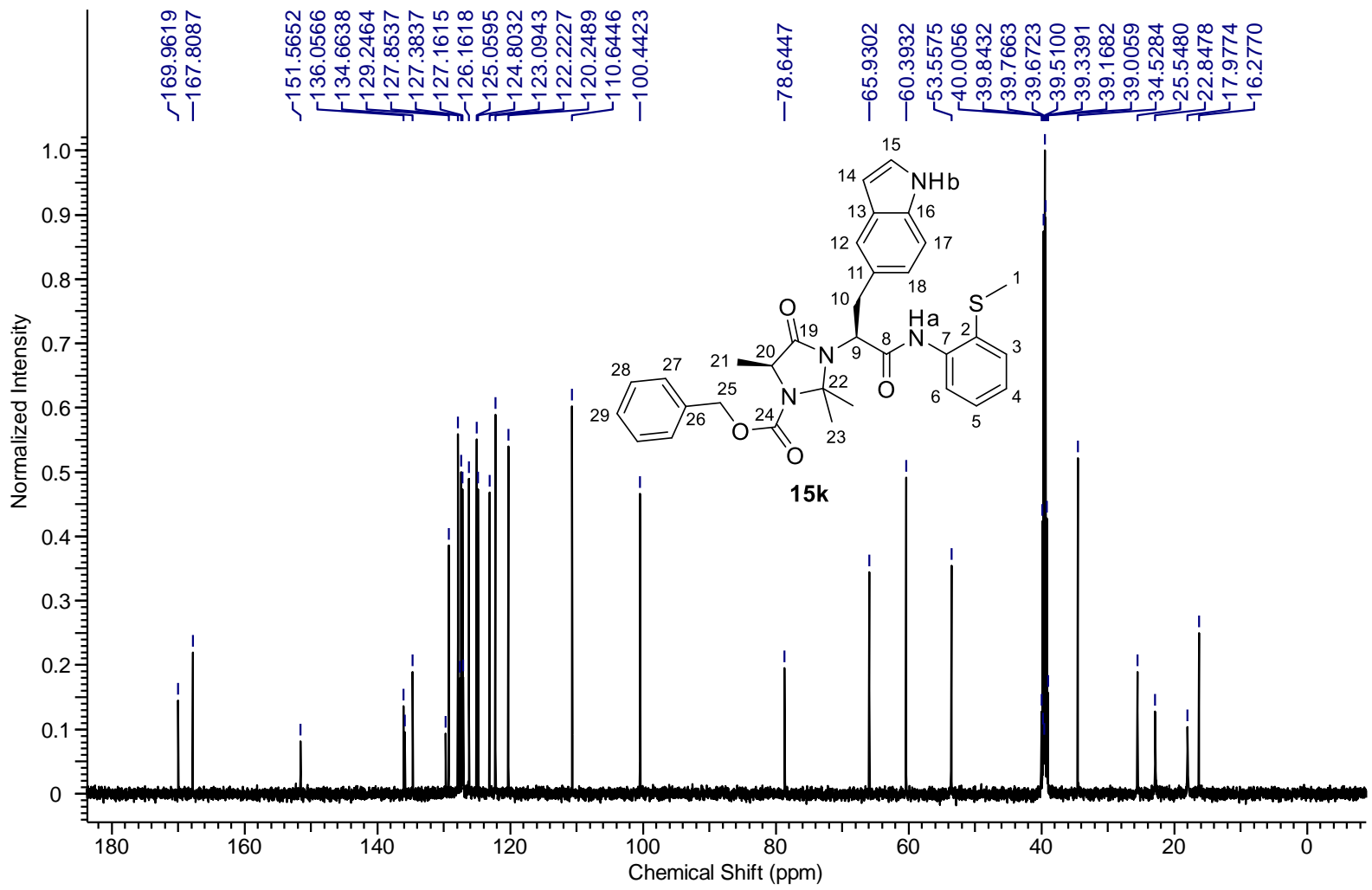


tert-Butyl 5-((S)-2-((S)-3-((benzyloxy)carbonyl)-2,2,4-trimethyl-5-oxoimidazolidin-1-yl)-3-((2-(methylthio)phenyl)amino)-3-oxopropyl)-1H-indole-1-carboxylate (15I)

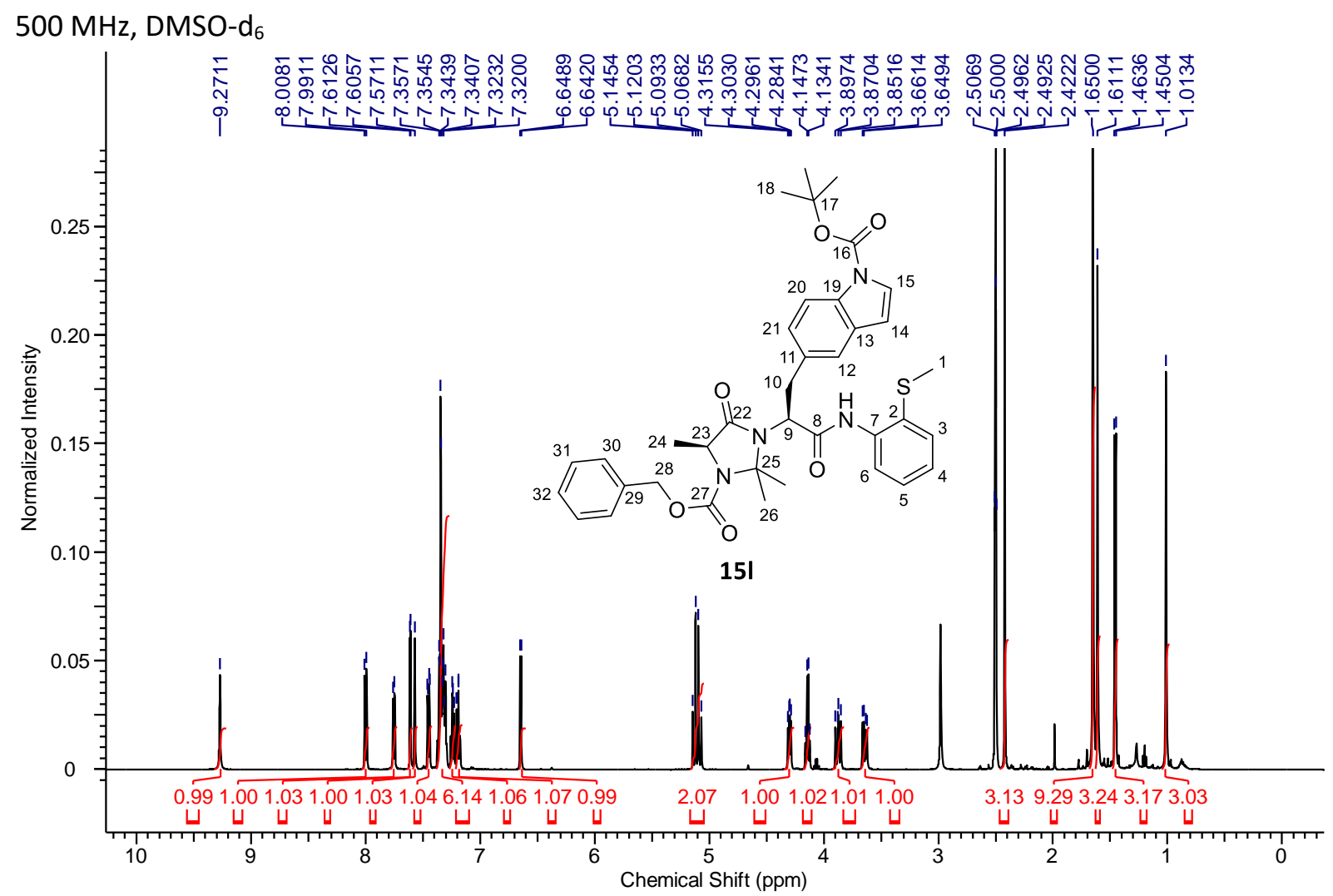

$125 \mathrm{MHz}, \mathrm{DMSO}-\mathrm{d}_{6}$

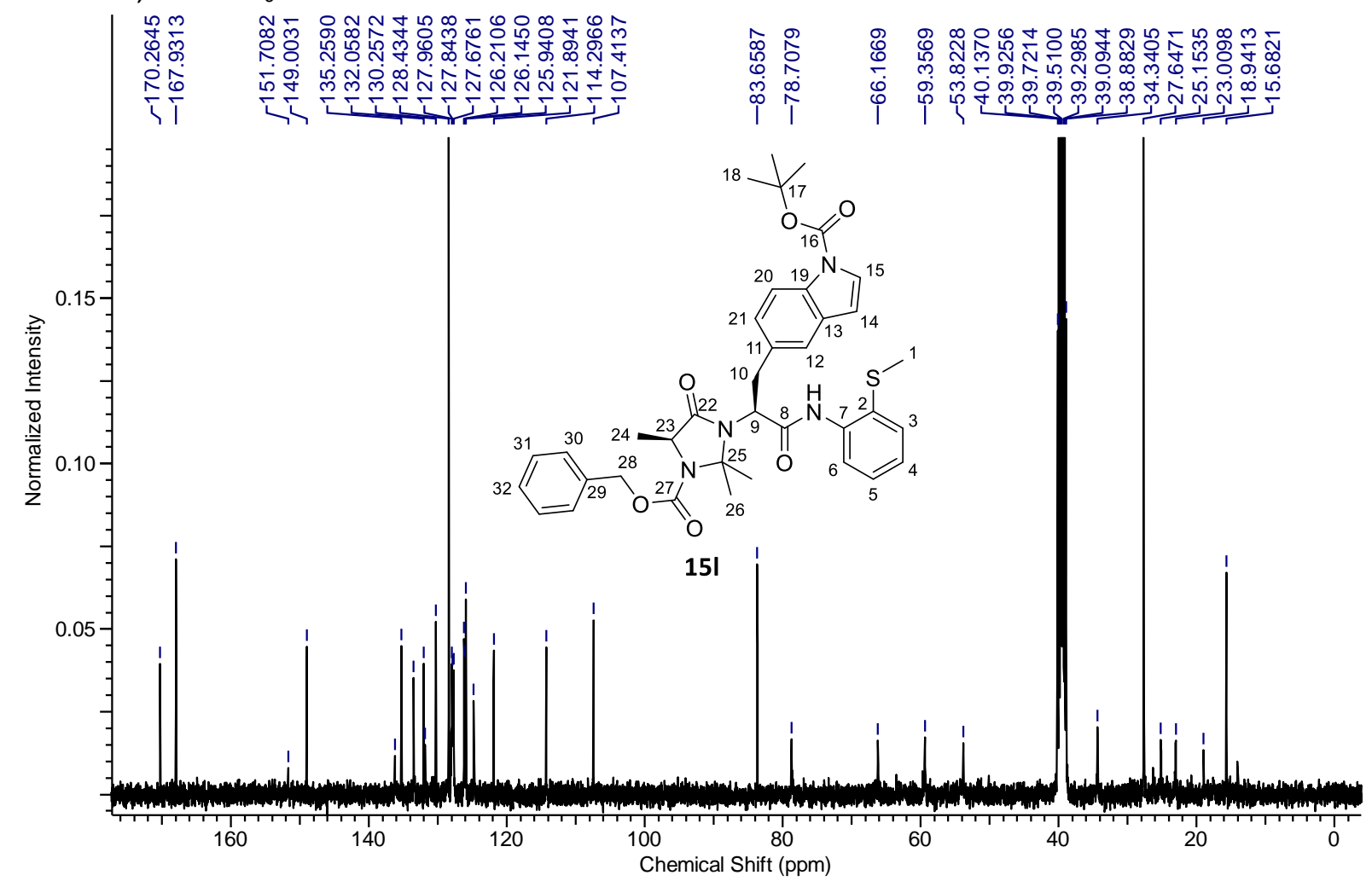


Benzyl (S)-2,2,5-trimethyl-3-((S)-1-((2-(methylthio)phenyl)amino)-1-oxo-3-(pyridin-2-yl)propan-2yl)-4-oxoimidazolidine-1-carboxylate $(15 \mathrm{~m})$

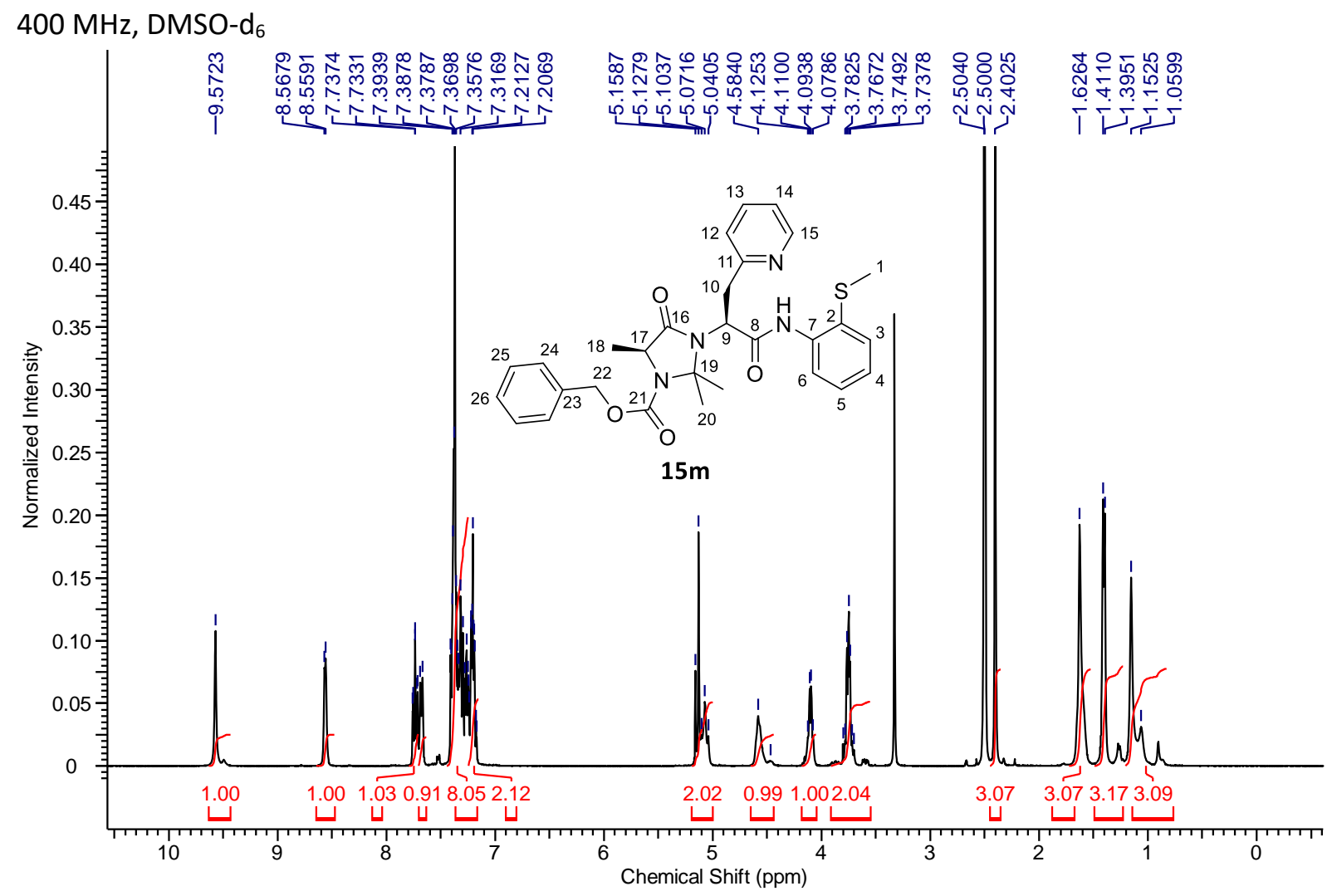

$100 \mathrm{MHz}, \mathrm{DMSO}-\mathrm{d}_{6}$

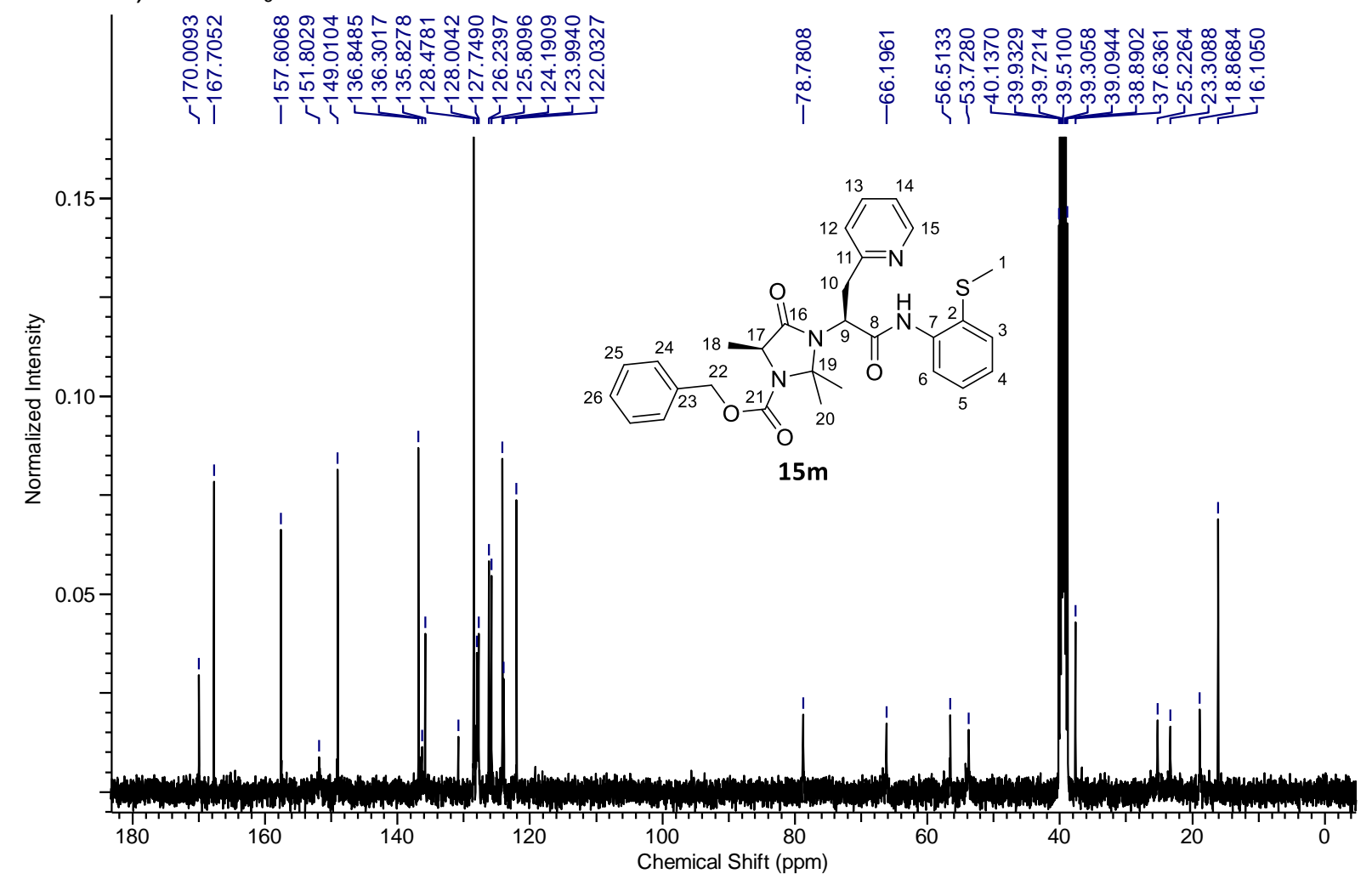


(S)-2-((S)-3-((Benzyloxy)carbonyl)-2,2,4-trimethyl-5-oxoimidazolidin-1-yl)-3-(p-tolyl)propanoic acid (16)
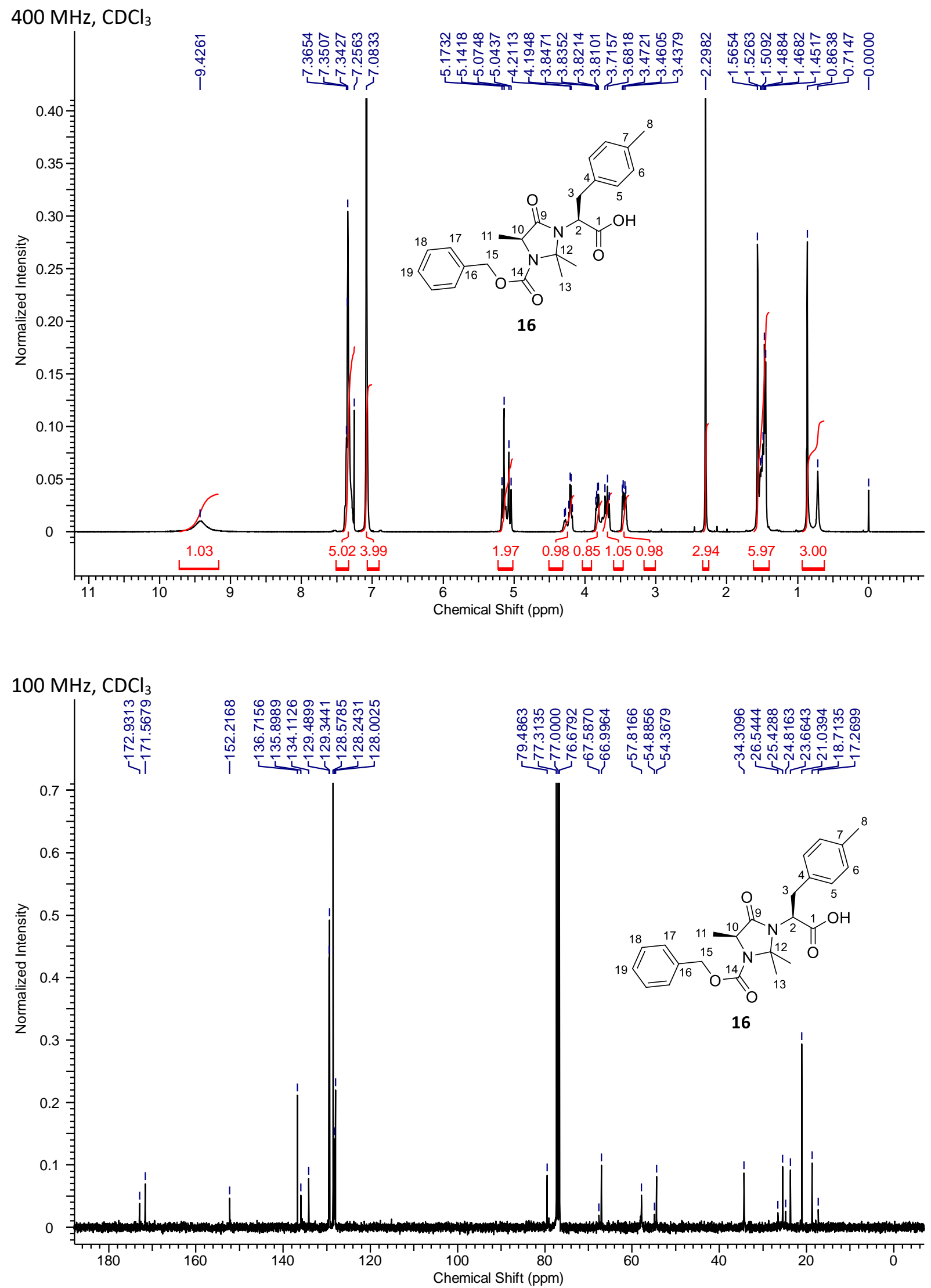
Benzyl (S)-3-((S)-1-((2-methoxy-2-oxoethyl)amino)-1-oxo-3-(p-tolyl)propan-2-yl)-2,2,5-trimethyl-4oxoimidazolidine-1-carboxylate (17)
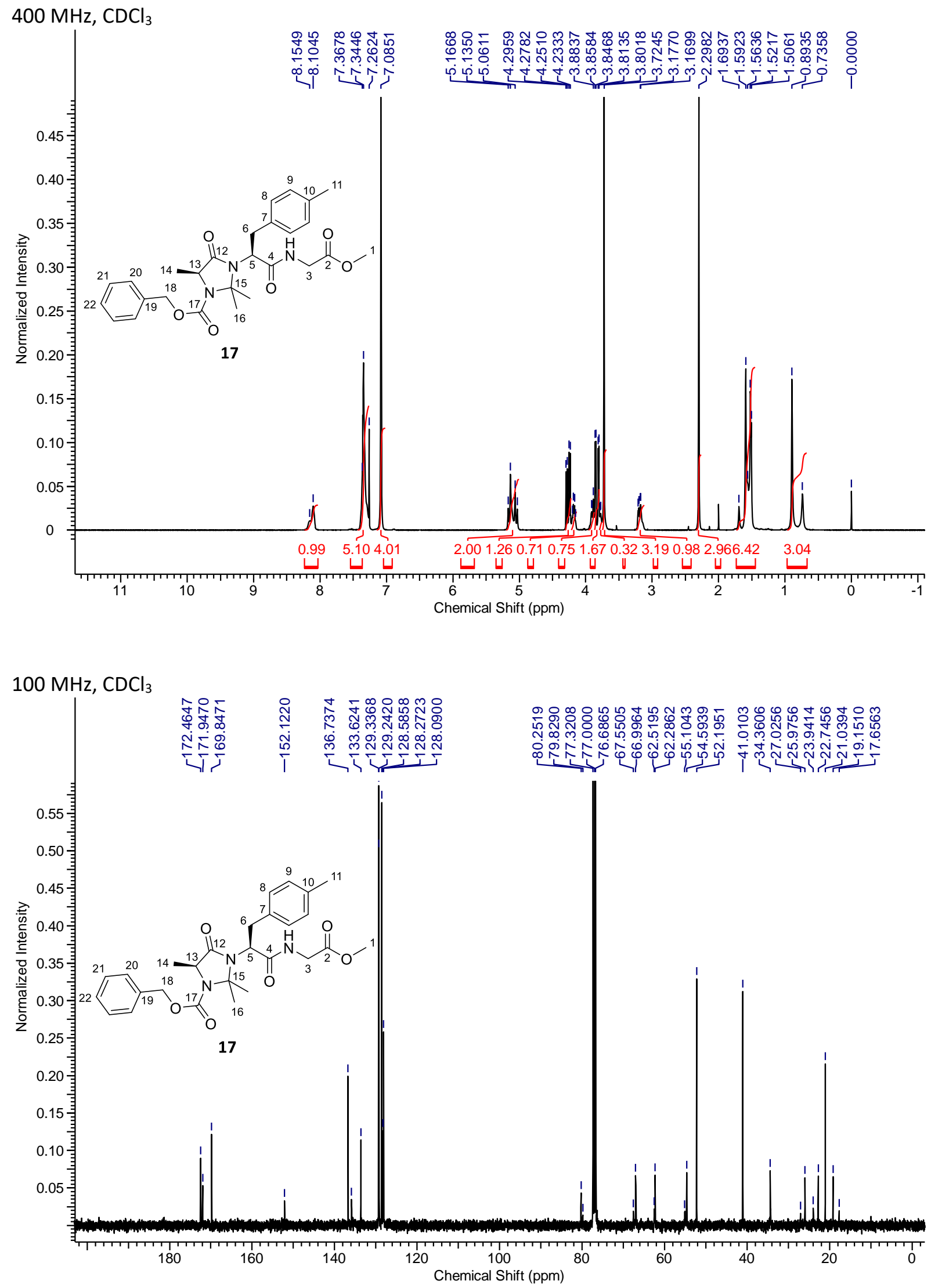
Methyl ((S)-2-((S)-2-((S)-3-(benzyloxy)-2-((tert-butoxycarbonyl)amino)propanamido)propanamido)3-(p-tolyl)propanoyl)glycinate (18)

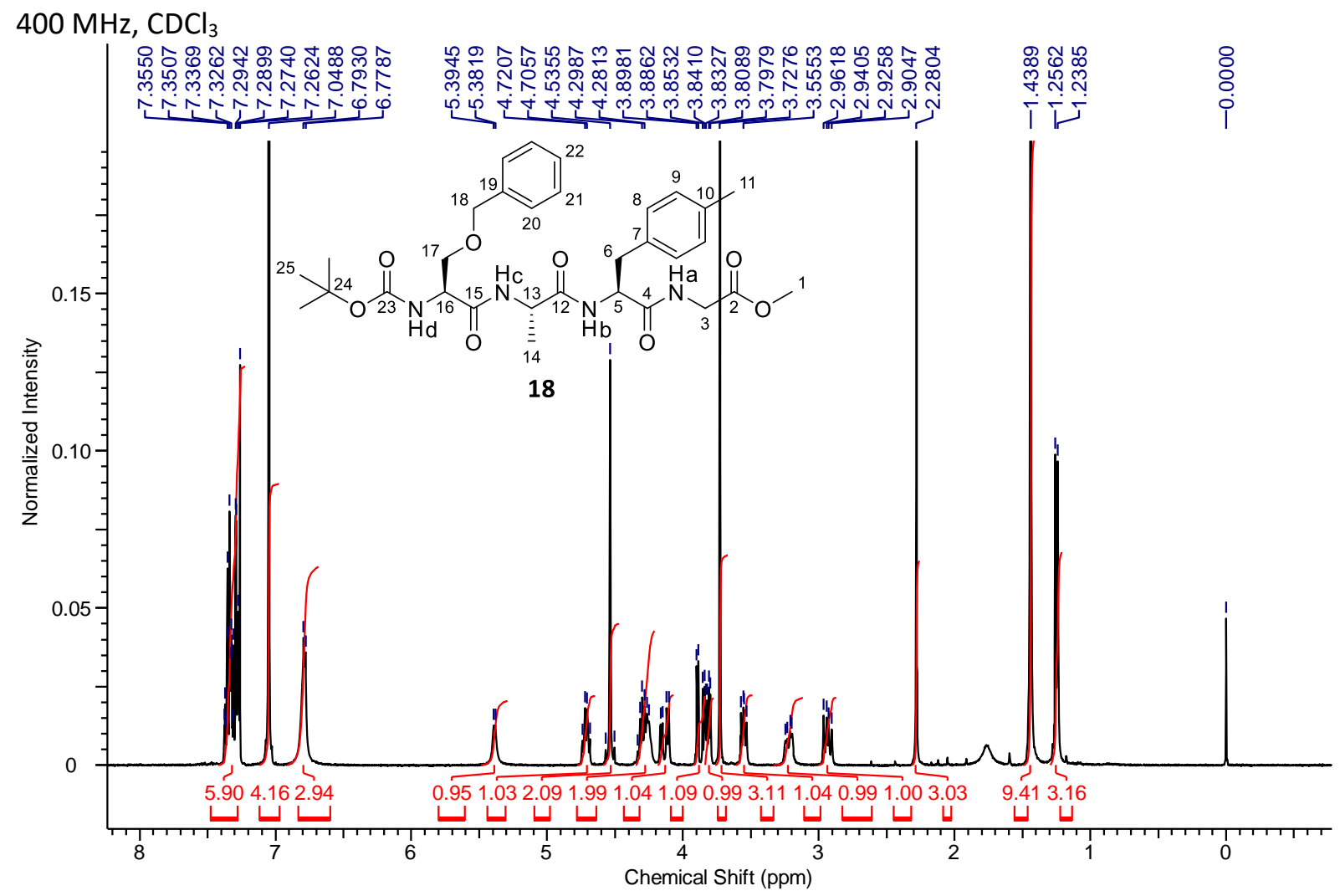

$100 \mathrm{MHz}_{2} \mathrm{CDCl}_{3}$

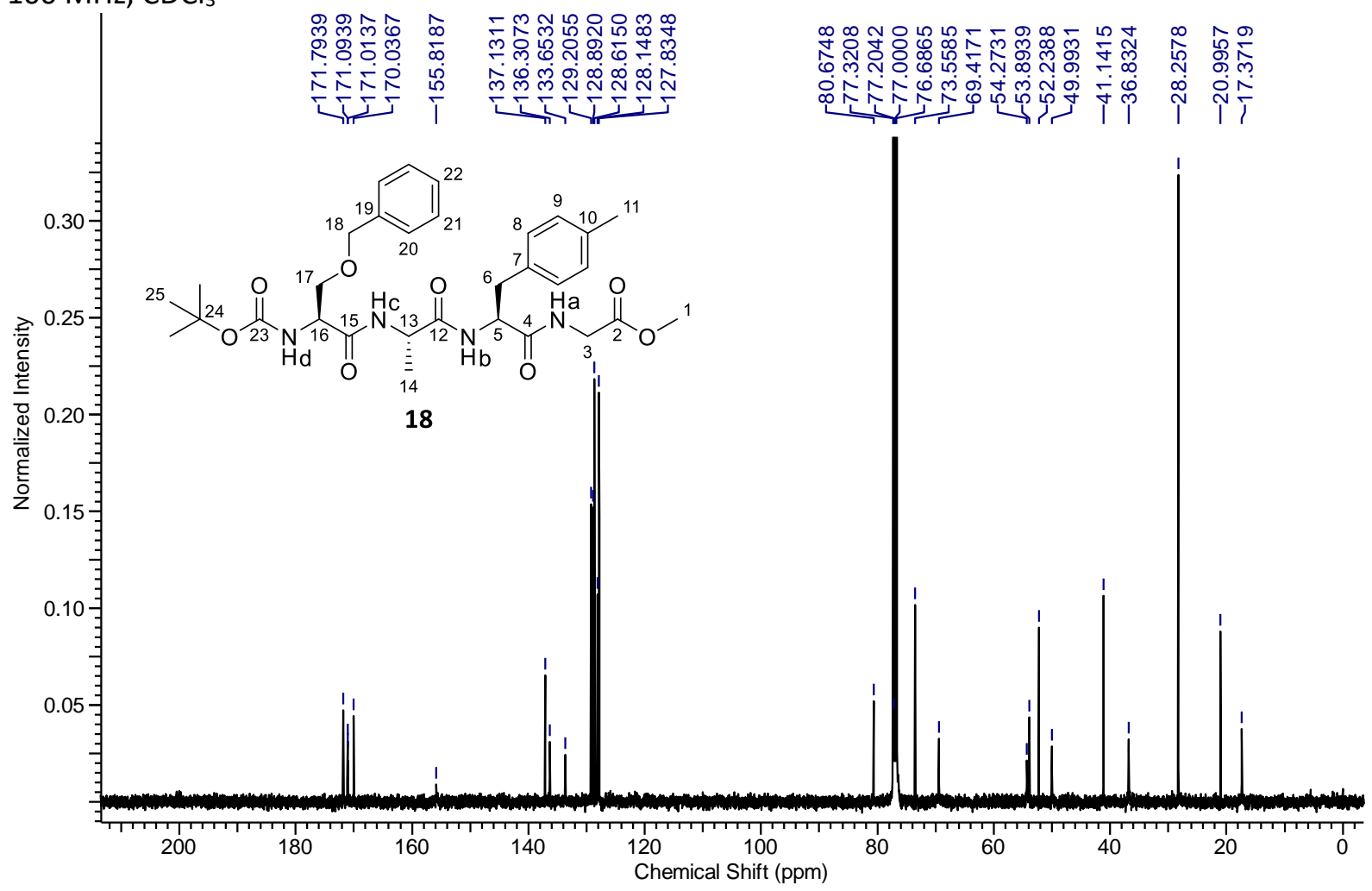

